\title{
Quantum Groups and Subfactors of Type B, C, and D
}

\author{
Hans Wenzl ${ }^{\star}$ \\ University of California, San Diego, La Jolla CA 92093, USA
}

\begin{abstract}
The main object of this paper is the study of a sequence of finite dimensional algebras, depending on 2 parameters, which appear in connection with the Kauffman link invariant and with Drinfeld's and Jimbo's $q$ deformation of Lie algebras of types $B, C$ and $D$. We determine for which parameters these algebras are semisimple. Moreover, we classify all unitary representations of the infinite braid group $B_{\infty}$ factoring through the inductive limit of these algebras. This yields new examples of irreducible subfactors of finite depth, whose indices are squares of $q$ dimensions of irreducible representations of sympletic and orthogonal groups. In the combinatorial description of these subfactors one naturally obtains truncated Weyl chambers (as for loop groups for a given level) and multiplicity coefficients of fusion rules for Wess-Zumino-Witten models.
\end{abstract}

We study a sequence of algebras $C_{f}, f \in \mathbf{N}$ which depend on 2 complex parameter $r$ and $q$. They are obtained as images of representations of the braid groups $B_{f}$. One way of describing them is by pictures indicating equivalence classes with respect to Kauffman's regular isotopy (see Sect. 3). Algebraically, they are given by generators $g_{1}, g_{2} \ldots g_{f-1}$ which satisfy, besides the braid relations, 2 more relations, namely the cubic equation $\left(g_{i}-r^{-1}\right)\left(g_{i}+q^{-1}\right)\left(g_{i}-q\right)=0$ and the contraction relation $p_{i} g_{i-1} p_{i}=\alpha p_{i}$, where $p_{i}$ is the spectral idempotent belonging to the characteristic value $r^{-1}$ of $g_{i}$ and $\alpha$ is a fixed scalar depending on $r$ and $q$ (see Sect. 3 for details).

These algebras naturally appear with a trace functional tr which will be referred to as Markov trace or as structure trace. It can either be derived from Kauffman's invariant or one can define it purely algebraically, which gives another proof for the Kauffman polynomial.

Unitary braid representations play an important role in the study of subfactors and in quantum field theory (see [W-1], [FRS] or [FFK]). So one of the main goals of this paper is the complete classification of unitary representations of $B_{\infty}$ which

* Supported in part by NSF grant \# DMS 88-05378 
factor over tr. To do so, let us first briefly describe the structure of $C_{f}$ in the semisimple case. One can show that the semisimplicity of $C_{f}$ is closely connected with the question whether $\operatorname{tr}$ is faithful on $C_{f-1}$, i.e. the bilinear form on $C_{f-1}$, defined by $\langle a, b\rangle=\operatorname{tr}(a b)$ is nondegenrate. If this is the case, $C_{f}$ can be written as the direct sum of an algebra $I_{f}$, which can be obtained from the inclusion $C_{f-2} \subset C_{f-1}$ by Jones' basic construction and Iwahori's Hecke algebra $H_{f}\left(q^{2}\right)$ of type $A_{f-1}$. So the structure of the $C_{f}$ 's can be determined inductively by its Bratteli diagram (see [BW] or the end of Sect. 2 in this paper).

If $\operatorname{tr}$ is not faithful, the same strategy can still be applied to determine the structure of the quotient $\pi_{\mathrm{tr}}\left(C_{f}\right)$ of $C_{f}=C_{f}(r, q)$ over the annihilator ideal of tr. In this case one has a direct sum of an algebra $\pi_{\mathrm{tr}}\left(I_{f}\right)$, whose structure can again be computed from the algebras $\pi_{\mathrm{tr}}\left(C_{f-2}\right) \subset \pi_{\mathrm{tr}}\left(C_{f-1}\right)$ and a quotient $\varrho_{f}\left(H_{f}\left(q^{2}\right)\right)$ of the Hecke algebra $H_{f}\left(q^{2}\right)$. So the structure of the $\pi_{\mathrm{tr}}\left(C_{f}\right)$ 's is known as soon as one knowns $\varrho_{f}\left(H_{f}\left(q^{2}\right)\right)$ for all $f \in \mathbf{N}$. All the necessary information can be encoded in a graph $\Gamma=\Gamma(r, q)$. Similar as for a Bratteli diagram, its vertices are labelled by the simple components of $\varrho_{f}\left(H_{f}\left(q^{2}\right)\right)$ and its edges describe the decomposition of the restriction of such a representation to $H_{f-1}$. Similar methods are used by Ocneanu and Goodman-de la Harpe-Jones to describe higher relative commutants of subfactors. So the main technical difficulty consists of determining the structure of the $\varrho_{f}\left(H_{f}\right)$ 's. For this we construct inductively semisimple quotients of $C_{f}$ which factor over an ideal which is contained in the annihilator of tr. After that one only needs to determine all those simple components of that quotient which are not annihilated by $\operatorname{tr}$. This is equivalent to the fact that $\operatorname{tr}(p) \neq 0$ for a minimal idempotent in that component. The computation of $\operatorname{tr}(p)$ is done by using representations of our algebras by Jimbo's explicit $R$ matrices, Weyl's character formulas analyticity arguments and inductively defined minimal idempotents.

Our main results are as follows:

(a) $C_{f}(r, q)$ is semisimple except possibly if $q$ is a root of unity or $r=q^{n}$ for some $n \in \mathbf{Z}$,

(b) $\pi_{\mathrm{tr}}$ is a unitary representation of $B_{\infty}$ if $q=e^{ \pm \pi i l l}$ and $r=q^{n}$ with $n, l \in \mathbf{Z}$ and $|n|<l$. Moreover, any unitary representation of $B_{\infty}$ which factors through $C_{\infty}(r, q)$ can be described by a subgraph of Young's lattice belonging to one of the following 3 cases [with some exceptions corresponding to $O(2)$ and $O(3)$ ]: Let $\lambda_{i}$ (respectively $\lambda_{j}^{\prime}$ ) denote the number of boxes in the $i^{\text {th }}$ row (respectively $j^{\text {th }}$ column) of the Young diagram $\lambda$. Then

(c1) if $q$ is a primitive $2 l^{\text {th }}$ root of unity and $2<n<l-2$,

$$
\Gamma\left(q^{n}, q\right)=\left\{\lambda, \lambda_{1}+\lambda_{2} \leqq l-n+1 \quad \text { and } \quad \lambda_{1}^{\prime}+\lambda_{2}^{\prime} \leqq n+1\right) \cup\left\{\left[l-n+1,1^{n-1}\right]\right\} .
$$

(c2) If $n<-1$, even and $l$ is odd,

$$
\Gamma\left(q^{n}, q\right)=\left\{\lambda, \lambda_{1}+\lambda_{2} \leqq-1-n \quad \text { and } \quad \lambda_{1}^{\prime} \leqq(l+n-1) / 2\right\} .
$$

(c3) If $n<-1$, odd and $l$ is even,

$$
\Gamma\left(q^{n}, q\right)=\left\{\lambda, \lambda_{1} \leqq(-1-n) / 2 \text { and } \lambda_{1}^{\prime} \leqq(l+n-1) / 2\right\} .
$$

Observe that these are exactly the restrictions for the possible representations of the classical part of loop groups for level $L$ representations in the orthogonal (c1), twisted type $A$ case (also referred to as $B C$ case) (c2) and symplectic case (c3) 
(see $[\mathrm{Kc}]$; I would like to thank Nolan Wallach for helping me in finding an interpretation for case (c2)).

Having these unitary representations available, it is fairly easy to construct new examples of subfactors of the hyperfinite $\mathrm{II}_{1}$ factor. They are of the form $\pi_{\mathrm{tr}}\left(B_{m+1, \infty}\right)^{\prime \prime} \subset \pi_{\mathrm{tr}}\left(B_{\infty}\right)^{\prime \prime}$, where $B_{m+1, \infty}$ is the subgroup of $B_{\infty}$ generated by $\sigma_{m+1}$, $\sigma_{m+2} \ldots$ and the double prime indicates the double commutant. We show that one can obtain for $r$ and $q$ as above and for $\lambda \in \Gamma(r, q)$ an irreducible subfactor of the hyperfinite $\mathrm{II}_{1}$ factor and compute their indices. Moreover, if $\lambda=[1]$, we also compute their higher relative commutants which shows that they are all of finite depth. It appears that this can also be done for the subfactors corresponding to other diagrams extending the ideas of $[\mathrm{GW}]$ to these cases. We expect that the multiplicities can be expressed by classical branching rules for tensor products and the action of an affine reflection group given by the Weyl group and a reflection about a hyperplane given by the highest root of the corresponding group as it was done in [GW]. As in type $A$, these numbers should be equal to the coefficients of the fusion rules of the Verlinde algebra for Wess-Zumino-Witten models (see also the concluding remarks).

Here is a more detailed account of the contents of this paper: The first 2 sections are of more introductory nature dealing with various algebraic tools needed later as well as reviewing and extending basic facts about Hecke algebras and Brauer algebras. Due to the connections of our algebras with several different areas, we present 3 different ways of introducing them. The first and in many ways simplest one comes from Kauffman's invariant of regular isotopy which can be used to define a trace functional tr on the group algebra of $B_{f}$. Then $C_{f}$ is just the quotient modulo the annihilator ideal of tr and the algebraic relations follow from the skein relations. This was the original way how a set of defining relations was discovered in $[\mathrm{BW}]$ and $[\mathrm{M}]$ and we review this exposition here simplifying various arguments in [BW].

The second way of defining $C_{f}$ is purely algebraic by generators and relations. The structure trace can be defined inductively on these algebras. This method allows one to determine the structure of the generic algebra $C_{f}$ (with the parameters viewed as indeterminates of a field of rational functions) using results about a specialization of it, Brauer's centralizer algebras.

In the fourth section, the methods of the previous one are extended to the algebras $C_{f}(r, q), r, q \in \mathbf{C}$. If $\operatorname{tr}$ is faithful and $q$ is not a root of unity, also the algebras $C_{f}(r, q)$ are semisimple. If tr is not faithful, one can determine the structure of $\pi_{\mathrm{tr}}\left(C_{f}(r, q)\right)$ from knowledge of the weight vector of $\mathrm{tr}$ in the generic case (i.e. from evaluating tr at minimal idempotents of the generic algebra). It is also shown that one can only expect an interesting * structure (by this we mean a representation on a Hilbert space where the images of the standard generators are normal operators) if either both $r$ and $q$ are real or both are of absolute value 1 . If this is the case, one does indeed obtain a $C^{*}$ structure if the trace is positive at all minimal idempotents of $\pi_{\mathrm{tr}}\left(C_{f}(r, q)\right)$.

All the necessary information about this is obtained by our $3^{\text {rd }}$ approach. It turns out that special solutions of the quantum Yang-Baxter equation, obtained from $q$-deformations of the enveloping algebra of the Lie algebra $s o_{n}$ (see [Ji-1] and [D]), can be used to construct representations of $C_{f}\left(q^{n-1}, q\right)$ (this observation appeared first in print in [Re]). The structure trace can now be easily obtained from a so-called product "state" generalizing work of Pimser and Popa who did this first in the context of subfactors for the Temperley-Lieb algebra (for the general 
case see $[\mathrm{Re}]$ or $[\mathrm{T}])$. The method goes as follows. One takes a special diagonal matrix $D$, closely related to half the sum of positive roots of $s o_{n}$ and defines the product "state" $\phi$ on the $f^{\text {th }}$ tensor power $M_{n}^{\otimes f}$ of $n \times n$ matrices by $\phi\left(a_{1} \otimes a_{2} \otimes \ldots \otimes a_{f}\right)=\prod_{i} \operatorname{Tr}\left(D a_{i}\right) / \operatorname{Tr}(D)$. We show that the GNS construction with respect to $\phi$ maps any subalgebra of the centralizer algebra of the $f$-fold tensor product representation of the quantum group onto a semisimple algebra. Moreover, in our case, if $q$ is not a root of unity, and $p$ is a minimal idempotent of $\Phi\left(C_{f}\left(q^{n-1}, q\right)\right) \operatorname{tr}(p)$ is equal to $\chi^{(\lambda)}(D) / \operatorname{Tr}(D)^{f}$, where $\chi^{(\lambda)}$ is the character of a representation of $O(n)$ appearing in the $f^{\text {th }}$ tensor power of its standard representation. This provides enough information to compute the weight vector also for the general algebra $C_{f}$ by an interpolation argument.

In the sixth chapter, this information is exploited to compute all possible semisimple representations and unitary representations of $C_{f}(r, q)$ which factor over tr. Using the methods of [W-1] one obtains from these unitary representations examples of subfactors which are constructed in Sect. 7. Moreover, we also compute their indices and higher relative commutants. Finally, we review some of the connections between Lie algebras and their quantizations, link invariants and subfactors in the concluding remarks.

A general overview of the results of this paper was given in a talk at the Congress of the IAMP, Swansea 1988 (see [W-4]) and more details were announced in [W-5]. This is a modified version of a preprint with the same title which has been circulated since summer 89. Related results have also appeared in [AGS].

\section{Preliminaries}

We are going to describe several types of finite dimensional algebras. For convenience we will mean by a semisimple algebra a finite direct sum of full matrix rings. Let for the moment $k$ be a field of characteristic 0 and let $k(x)$ denote the field of rational functions over $k$. The algebra of all $n \times n$ matrices is denoted by $M_{n}(k)$ or just $M_{n}$. So if $A$ and $B$ are semisimple $k$ algebras, we can write them as $A=\oplus A_{i}$ and $B=\oplus B_{j}$ with $A_{i} \cong M_{a_{i}}(k)$ and $B_{j} \cong M_{b_{j}}(k)$ for appropriate natural numbers $a_{i}$ and $b_{j}$.

If $A$ is a subalgebra of $B$, any simple $B_{j}$ module is also an $A$ module. Let $g_{i j}$ be the number of simple $A_{i}$ modules in its decomposition into simple $A$ modules. The matrix $G=\left(g_{i j}\right)$ is called the inclusion matrix for $A \subset B$.

The inclusion of $A$ in $B$ is conveniently described by a so-called Bratteli diagram. This is a graph with vertices arranged in 2 lines. In one line, the vertices are in 1-1 correspondence with the minimal direct summands $A_{i}$ of $A$, in the other one with the summands $B_{j}$ of $B$. Then a vertex corresponding to $A_{i}$ is joined with a vertex corresponding to $B_{j}$ by $g_{i j}$ edges. If $A$ and $B$ have the same identity, there is an easy way of computing the square root of the dimension $b_{j}$ of $B_{j}$. We just add up all the square roots of the dimensions of $A_{i}$ 's to which $B_{j}$ is joined by edges (with multiplicities).

We can also interpret the numbers $g_{i j}$ in the following way: Let $p_{i}$ be a minimal idempotent of $A_{i}$ and let $p_{i}=\sum q_{m}$, where the $q_{m}$ 's are mutually orthogonal minimal idempotents of $B$. This decomposition is not unique in general. But for any such decomposition there will be exactly $g_{i j}$ idempotents in $B_{j}$. As an easy consequence 
we obtain that

$$
p_{i} B p_{i} \cong \underset{j}{\oplus} M_{g_{i j}} .
$$

We will describe, as an example, the inclusion of $k S_{f-1}$ in $k S_{f}$, where $k S_{f-1}$ and $k S_{f}$ are the group algebras of the corresponding symmetric groups. Let for $f \geqq 0 \Lambda_{f}$ be the set of all Young diagrams (or Ferrer's diagrams) with $f$ nodes. We will write a specific diagram $\lambda$ as an $m$-tupel $\left[\lambda_{1}, \ldots, \lambda_{m}\right]$, where $\lambda_{i}$ is the number of boxes in the $i^{\text {th }}$ row. The empty Young diagram in $\Lambda_{0}$ is denoted by [0]. We will mean here by the Young lattice the infinite graph whose vertices are labelled by all Young diagrams such that 2 vertices are connected by an edge if and only if the corresponding diagrams differ by exactly one box (i.e. one of them can be obtained by adding a box to the other one). It is well-known that the simple components $k S_{f, \lambda}$ of $S_{f}$ are labeled by diagrams $\lambda$ with $f$ boxes. So the Bratteli diagram for $k S_{f-1} \subset k S_{f}$ is the subgraph of the Young lattice with the vertices labelled by diagrams with $f-1$ and $f$ boxes (and the edges between these vertices). The inclusion diagram for $k S_{2} \subset k S_{3}$ is shown in the upper half of Fig. 1 (with $\left[1^{m}\right]=[1, \ldots, 1](m$ times)).

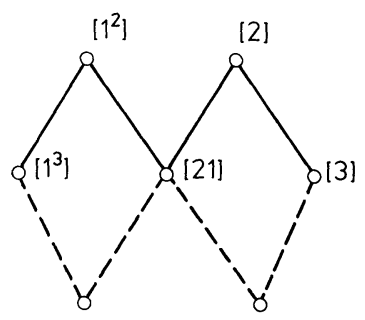

Fig. 1

An important role will be played by traces, i.e. functionals $\operatorname{tr}: B \rightarrow k$ such that $\operatorname{tr}(a b)=\operatorname{tr}(b a)$ for all $a, b \in B$. As there is up to scalar multiples only one trace on $M_{n}(k)$, any trace $\operatorname{tr}$ on $B=\oplus B_{j}$ is completely determined by a vector $\left(t_{j}\right)$, where $t_{j}$ $=\operatorname{tr}\left(p_{j}\right)$ and $p_{j}$ is a minimal idempotent of $B_{j}$. The annihilator ideal $J$ of $\operatorname{tr}$ is defined to be

$$
J=\{b \in B, \operatorname{tr}(a b)=0 \text { for all } a \in B\} .
$$

A trace $\operatorname{tr}$ on $B$ is called nondegenerate it $J=0$, or, equivalently, if for any $b \in B$ there is a $b^{\prime} \in B$ such that $\operatorname{tr}\left(b b^{\prime}\right) \neq 0$. It is easy to check that tr is nondegenrate if and only if $t_{j} \neq 0$ for each $j$. The representation $\pi_{\mathrm{tr}}$ of $B$ is defined on $B / J$ by left multiplication. Because of the trace property, it is easy to check that

$$
\pi_{\mathrm{tr}}(B) \cong B / J \text {. }
$$

Let us recall that if $\operatorname{tr}$ is a nondegenrate trace on $B$, the map $b \in B \mapsto \operatorname{tr}(b.) \in B^{*}$ is an isomorphism between $B$ and its dual $B^{*}$ (where as usual $\operatorname{tr}(b$.) denotes the map $x \mapsto \operatorname{tr}(b x)$ ). Let $\operatorname{tr}$ be nondegenerate on both $A$ and $B$. Using the isomorphism above for $A$ and $A^{*}$, we obtain for every $b \in B$ a necessarily unique $\varepsilon_{A}(b) \in A$ such that $\operatorname{tr}(b .)_{\mid A}=\operatorname{tr}\left(\varepsilon_{A}(b) .\right)_{\mid A}$. The linear map $\varepsilon_{A}: B \rightarrow A, b \mapsto \varepsilon_{A}(b)$ is called the trace preserving conditional expectation from $B$ onto $A$, where the element $\varepsilon_{A}(b) \in A$ is uniquely determined by the equation

$$
\operatorname{tr}\left(\varepsilon_{A}(b) a\right)=\operatorname{tr}(b a) \text { for all } a \in A .
$$


We obtain from this equation and the faithfulness of tr the following properties of $\varepsilon_{A}$ :

(a) $\varepsilon_{A}\left(a_{1} b a_{2}\right)=a_{1} \varepsilon_{A}(b) a_{2}$ for all $a_{1}, a_{2} \in A$ and $b \in B$ and in particular $\varepsilon_{A}(a)=a$ for all $a \in A$.

(b) $\varepsilon_{A}$ is nondegenerate, i.e. for all $0 \neq b \in B$ there is $b_{1}, b_{2} \in B$ such that $\varepsilon_{A}\left(b_{1} b\right) \neq 0$ and $\varepsilon_{A}\left(b b_{2}\right) \neq 0$.

Later on, we shall also consider the case when both $A$ and $B$ are $C^{*}$ algebras, i.e. there exists a faithful representation of $B$ on a Hilbert space such that both $B$ and $A$ are closed under the $*$ operation which assigns to a linear operator its adjoint. A trace $\operatorname{tr}$ is called positive if $\operatorname{tr}\left(b^{*} b\right) \geqq 0$ for all $b \in B$. In the finite dimensional case one has

$\operatorname{tr}$ is positive if and only if all components of the weight vector are nonnegative.

If all components of the weight vector of the trace are positive, one has an inner product on $B$ defined by

$$
\langle a, b\rangle=\operatorname{tr}\left(b^{*} a\right) .
$$

In this case, the conditional expectation $\varepsilon_{A}$ can be interpreted as the orthogonal projection onto the subspace $A \subset B$. It has the following additional properties:

and

$$
\varepsilon_{A}\left(b^{*}\right)=\varepsilon_{A}(b)^{*}
$$

$$
\varepsilon_{A}\left(b^{*} b\right) \geqq 0 \quad \text { for all } b \in B \text {. }
$$

Let again $A$ and $B$ be arbitrary finite dimensional semisimple algebras and let $\operatorname{tr}$ be a nondegenerate trace on both $A$ and $B$. We will moreover assume that $B$ is contained in an algebra $C$ and that there is an element $e \in C$ such that

(a) $e^{2}=e$,

(b) $e b e=e \varepsilon_{A}(b)=\varepsilon_{A}(b) e$ for all $b \in B$,

(c) The map $a \in A \mapsto a e$ is an injective homomorphism with $1 e=e$.

A important example for such a situation is Jones' basic construction (see [Jo-1, Sect. 3.1]): Let $B$ be represented via left regular representation on itself. For convenience, the isomorphic image of $B$ in this representation will also be denoted by $B$. If $B$ is regarded as representation space, it will be denoted by $B_{\xi}$ and its elements by $b_{\xi}$ with $b \in B$. We take as $C$ the set $L\left(B_{\xi}\right)$ of all linear maps on $B_{\xi}$. As in [Jo-1] we define an idempotent $e_{A}$ on $B_{\xi}$ by $e_{A} b_{\xi}=\varepsilon_{A}(b)_{\xi}$. It follows from this definition that

$$
\left(e_{A} b e_{A}\right) b_{\xi}^{\prime}=\left(e_{A} b\right) \varepsilon_{A}\left(b^{\prime}\right)_{\xi}=\left(\varepsilon_{A}(b) e_{A}\right) b_{\xi}^{\prime}=\left(e_{A} \varepsilon_{A}(b)\right) b_{\xi}^{\prime} \text { for all } b^{\prime} \in B
$$

Using again (1.7), (a) we show that $e_{A}$ is an idempotent and that $\left(a e_{A}\right) b_{\xi}=\left(e_{A} a\right) b_{\xi}$ for all $a \in A$ and $b \in B$. Finally, the equation $\left(a e_{A}\right) 1_{\xi}=a_{\xi}$ shows that the map $a \in A \mapsto a e_{A}$ is injective. The algebra $\left\langle B, e_{A}\right\rangle$ will be referred to as Jones' basic construction for $A \subset B$.

We have the following results for the set-up in (1.7) (see [J-1] and [W-2, Proposition (1.2) and Theorem (1.3)]): 
Theorem 1.1. Let $A, B, e, e_{A}$, tr and $\varepsilon_{A}$ be as above. Then

(a) The algebra $\left\langle B, e_{A}\right\rangle$ is isomorphic to the centralizer of $A$ on $B_{\xi}$, which is denoted by End $_{A} B$. In particular, it is semisimple.

(b) There is a 1-1 correspondence between the simple components of $A$ and $\left\langle B, e_{A}\right\rangle$ such that if $p \in A_{i}$ is a minimal idempotent, $p e_{A}$ is a minimal idempotent of $\left\langle B, e_{A}\right\rangle$. Under this correspondence, the inclusion matrix for $B \subset\left\langle B, e_{A}\right\rangle$ is the transposed $G^{t}$ of the inclusion matrix for $A \subset B$.

(c) $\left\langle B, e_{A}\right\rangle=B e_{A} B$.

(d) $\langle B, e\rangle$ is a direct sum of full matrix rings which decomposes as

$$
\langle B, e\rangle \cong\left\langle B, e_{A}\right\rangle \otimes \widetilde{B},
$$

where $\widetilde{B}$ is an algebra isomorphic to a subalgebra of $B$. In particular, the ideal generated by $e$ is isomorphic to the semisimple algebra $\left\langle B, e_{A}\right\rangle$.

Observe that statement (b) of the last theorem can be used to compute the structure of End ${ }_{A} B$ by reflecting the Bratteli diagram for $A \subset B$ about the line of $B$ (see Fig. 1 for our example $k S_{2} \subset k S_{3}$ ).

One of the main problems dealt with later is the question whether certain representations can be unitarized. For this, we assume $A$ and $B$ to be finite dimensional $C^{*}$ algebras. The question is under which conditions can one find a $C^{*}$ structure on $\langle B, e\rangle$ compatible with the one of $B$ such that $e$ is a selfadjoint projection. In such a case we shall just say that there exists a $\mathrm{C}^{*}$ representation of $e$. By the theorem above, it is enough to consider the case $e=e_{A}$.

Lemma 1.2. Let $A \subset B$ be finite dimensional $C^{*}$ algebras and let $\mathrm{tr}$ be a trace which is faithful on both $A$ and $B$. Let $\tilde{z}_{i}$ be the minimal central projection of $\left\langle B, e_{A}\right\rangle$ corresponding to the minimal central idempotent $z_{i} \in A$. Then there exists a $\mathrm{C}^{*}$ representation of $\tilde{z}_{i} e_{A}$ if and only if $\operatorname{tr}\left(\tilde{z}_{j}\right) \operatorname{tr}\left(z_{i}\right)>0$ for all minimal central projections $\tilde{z}_{j}$ of $B$ for which $\tilde{z}_{j} z_{i} \neq 0$.

In particular, if the inclusion matrix for $A \subset B$ is connected and if $\operatorname{tr}(1)>0, \operatorname{tr}$ has to be positive.

Proof. Assume that for a given minimal central projection $\tilde{z_{i}} \in\left\langle B, e_{A}\right\rangle$ we have $\operatorname{tr}\left(\tilde{z}_{j}\right) \operatorname{tr}\left(z_{i}\right)>0$ for all minimal central projections $\tilde{z}_{j}$ of $B$ for which $\tilde{z}_{j} z_{i} \neq 0$. By part (b) of Theorem (1.1) we have $\tilde{z}_{i} B \subset \sum_{\tilde{z}_{j} z_{i} \neq 0} \tilde{z}_{j} B$. Hence by replacing tr by - tr if necessary, we can assume that $\operatorname{tr}$ induces a positive definite inner product on $\bar{z}_{i} B$. Now $\bar{z}_{i} e_{A}$ is just the orthogonal projection with respect to this inner product onto the subspace $\bar{z}_{i} A$ of $\bar{z}_{i} B$ by the remark after (1.6).

On the other hand assume that there exists a central projection $\tilde{z}_{j}$ in $B$ such that $\operatorname{tr}\left(z_{i}\right) \operatorname{tr}\left(\tilde{z}_{j}\right)<0$ and $\tilde{z}_{j} z_{i} \neq 0$. Let $q$ be a minimal projection in $z_{i} A$ and let $p$ be a minimal projection in $z_{i} \tilde{z}_{j} B$ which is majorized by $q$. Let $\varepsilon_{A}$ be the trace preserving conditional expectation onto $A$. It follows easily that $\varepsilon_{A}(p)=\alpha q$ where $\alpha=\operatorname{tr}(p) / \operatorname{tr}(q)>0$. But then $e_{A} p e_{A}=\varepsilon_{A}(p) e_{A}=\alpha q e_{A}$. Obviously, the element on the right-hand side is not positive wich it would be if $e_{A}$ were so.

In the following complex algebras will be considered which depend on one or 2 complex parameters. It is sometimes more convenient to view these algebras as algebras over a field of rational functions with the parameters replaced by the 
indeterminates of the field. The following statements relate the "polynomial algebras" [i.e. the algebras over $\mathbf{C}(x)$ ] to the "concrete algebras" (i.e. the algebras over C).

Let more generally $A$ be a finite dimensional $k(x)$ algebra, where $k$ is a field of characteristic 0 , with a given basis $b_{1}, b_{2}, \ldots b_{d}$. Let the multiplication of 2 basis elements be given by

$$
b_{r} b_{s}=\sum_{i=1}^{d} \alpha_{r, s, i} b_{i}
$$

where $\alpha_{r, s, i}$ are rational functions over $k$ for $r, s, i=1,2, \ldots d$. If $q \in k$ is such that $q$ is not a pole for any of these rational functions, we can define a $d$ dimensional $k$ algebra $A(q)$ with basis $b_{1}(q), \ldots b_{d}(q)$ and multiplication

$$
b_{r}(q) b_{s}(q)=\sum_{i=1}^{d} \alpha_{r, s, i}(q) b_{i}(q)
$$

Let $k[x]_{q}$ be the localization of the polynomial ring $k[x]$ at $(x-q)$, i.e. $k[x]_{q}$ consists of all those rational functions whose denominators do not have a zero at $q$ and let $A_{q}$ be the $k[x]_{q}$-linear span of $\left\{b_{1}, b_{2}, \ldots b_{d}\right\}$. Then one can define for each $a \in A_{q}$ the element $a(q)=\sum \beta_{i}(q) b_{i}(q) \in A(q)$. It is easy to see that the map

$$
\Phi: a \in A_{q} \mapsto a(q) \in A(q)
$$

defines a surjective ring homomorphism from $A_{q}$ onto $A(q)$.

The following presumably well-known facts show some of the connections between $A$ and $A(q)$ (see [W-2, Lemma 2.3] and [W-3, Lemmas 5 and 6])

Proposition 1.3. Let $p \in A$ be an idempotent and let $q \in k$ be such that $A(q)$ and $p(q)$ are well-defined.

(a) $\operatorname{dim}_{k} p(q) A(q) p(q)=\operatorname{dim}_{k(x)} p A p$.

(b) Let $B \subset A$ be a subalgebra and let $B(q)=\Phi\left(B \cap A_{q}\right)$. If there exists $q \in k$ such that $\operatorname{dim}_{k} B(q)=\operatorname{dim}_{k(x)} B$ and $B(q)$ is semisimple, then also $B$ is semisimple. In this case $B \cong B(q) \otimes k(x)$ and $z(q)$ is well-defined for any central idempotent $z \in B$.

Let $\phi$ be a $k(x)$ valued functional on $A$. If $\phi\left(b_{i}\right) \in k[x]_{q}$ for $i=1,2, \ldots d$, one obtains a well-defined functional on $A(q)$, also denoted by $\phi$, by

$$
\phi(a(q))=\phi(a)(q) \text {. }
$$

Lemma 1.4. Assume that $A$ has a decomposition $A=\bigoplus_{j=1}^{r} A_{j}$ with $A_{j} \cong M_{a_{j}}(k(x))$. Let $\operatorname{tr}$ be a $k(x)$ valued trace on $A$ given by the weight vector $\left(P_{j}\right)_{j}, P_{j} \in k(x)$.

(a) If $P_{j}(q)$ is well-defined for $j=1,2, \ldots r$, then $\pi_{\mathrm{tr}}(A(q))$ is semisimple.

(b) Let $\in A$ be an idempotent which can be written as a sum of minimal idempotents $e_{i}$ all of which are in simple components $A_{j}$ of $A$ for which $P_{j}(q)=0$. If $e(q)$ is welldefined, then $\pi_{\mathrm{tr}}(e(q))=0$.

(c) Let $A_{j}$ be a direct summand of $A$ with corresponding central idempotent $z_{j} . A_{j}(q)$ is a well-defined simple direct summand of $A(q)$ with central idempotent $z_{j}(q)$ if

$(\alpha)$ there exists a minimal idempotent $p \in A_{j}$ such that $p(q)$ is well-defined and

$(\beta)$ there exists a matrix representation $\pi$ of $A_{j}$ such that also its evaluation at $x=q$ is well-defined and simple. 
Proof. (a) We identify $A$ with a faithful matrix representation over $k(x)$. Let $a, b \in A$ such that $a(q)$ is in the radical of $A(q)$.

As $a b(q)$ is nilpotent, all eigenvalues of the matrix representing $a b$ have to be divisible by $(x-q)$. Let $(a b)_{j}$ be the matrix for $a b$ in $A_{j}$ and let $\operatorname{Tr}$ be the usual trace for matrices, i.e. the sum of the diagonal elements. By the remarks above $(x-q)$ divides $\operatorname{Tr}\left((a b)_{j}\right)$. But as $\operatorname{tr}(a b)=\sum_{j}^{r} P_{j} \operatorname{Tr}\left((a b)_{j}\right)$, and as $P_{j} \in k[x]_{q},(x-q)$ also divides $\operatorname{tr}(a b)$. Hence $\operatorname{tr}(a(q) b(q))=0$ for all $b \in A_{q}$. As $\Psi$ is surjective, this implies $a(q) \in \operatorname{ker}\left(\pi_{\mathrm{tr}}\right)$.

The proof of (b) uses basically the same technique as the one of (a). If $a(q)$ is well-defined for some $a \in A$, then so is $(e a e)(q)$ and its eigenvalues. Hence the eigenvalues of eae do not have poles at $x=q$. It follows from this and the given assumptions that

$$
\operatorname{tr}(a e)(q)=\sum_{j}\left(P_{j} \operatorname{Tr}\left((a e)_{j}\right)\right)(q)=0 .
$$

(c) By $(\alpha)$ and $(\beta)$ there exist elements $a_{r}, b_{s} \in A_{q}$ such that $\left\{\pi\left(a_{r} p b_{s}\right\}\right.$ forms a set of matrix units. As $p \in A_{j}$ so is $a_{r} p b_{s}$ and it is well-defined at $x=q$ by assumption for all choices of $r$ and $s$. So having enough matrix units, one sees that $A_{j}(q)$ is well-defined and semisimple and, in particular, $z_{j}(q)=\sum a_{r} p b_{r}(q)$ is well-defined.

Corollary 1.5. The statements above also hold if $A$ is an algebra over $\mathbf{C}\left(x_{1}, x_{2}, \ldots x_{m}\right)$.

Proof. The proofs go by induction on $m$ with $m=1$ already shown. The step $m-1 \rightarrow m$ follows similarly from the lemmas above by setting $k=\mathbf{C}\left(x_{1}, x_{2}, \ldots x_{m-1}\right)$.

\section{Hecke Algebras and Brauer Algebras}

The main subject of this paper are algebras which are homomorphic images of the braid groups. The braid group $B_{f}$ can be defined topologically (see e.g. [Bi] or Sect. 3) or algebraically by generators $\sigma_{1}, \sigma_{2}, \ldots \sigma_{f-1}$ and relations

$$
\begin{aligned}
& \sigma_{i} \sigma_{i+1} \sigma_{i}=\sigma_{i+1} \sigma_{i} \sigma_{i+1} \text { for } i=1,2, \ldots f-2 \text { and } \\
& \sigma_{i} \sigma_{j}=\sigma_{j} \sigma_{i} \text { for }|i-j| \geqq 2 .
\end{aligned}
$$

We will also need the fact that the map $v_{f}$ given by $v_{f}\left(\sigma_{i}\right)=\sigma_{f-i}$ for $i=1,2, \ldots f-1$ extends to an inner automorphism of $B_{f}$ which is denoted by the same symbol (see e.g. [Bi, Lemma 2.5.1]).

Let $H_{f}$ be the Hecke algebra of type $A$ over $\mathbf{C}(x)$, the field of rational functions over $\mathbf{C}$, given by generators $g_{1}, g_{2}, \ldots g_{f-1}$ which satisfy the relations (B1), (B2) (with $g_{i}$ for $\sigma_{i}$ ) and

(H) $g_{i}^{2}=(x-1) g_{i}+x$ for $i=1,2, \ldots$.

If $q$ is a complex number, we denote by $H_{\infty}(q)$ the complex Hecke algebra generated by generators and relations as above where the variable $x$ is replaced by $q$. It can be shown in both cases (see e.g. [Bk], p 54-56, [H] or [W-1]) that $H_{f}$ is spanned linearly by the $n$ ! elements of the form

$$
b_{1} b_{2} \ldots b_{f}, \quad \text { where } \quad b_{i}=g_{j_{i}}, g_{j_{i}+1} \ldots g_{i-1}, \quad 1 \leqq j_{i} \leqq i-1, \quad \text { or } \quad b_{i}=1 \text {. }
$$


If $q=1, g_{1}, g_{2} \ldots g_{f-1}$ satisfy the relations of a set of simple reflections of $S_{f}$. Hence it follows from the results of the previous section that $H_{f}$ and $H_{f}(q)$ for all but finitely many values of $q$ are semisimple and have the same decomposition into full matrix rings as $\mathbf{C} S_{f}$. It is shown explicitly in [W-1] that $H_{f}(q)$ is not semisimple only for $q$ a primitive $k^{\text {th }}$ root of unity with $k=2,3, \ldots f$ or for $q=0$.

So whenever $H_{f}(q)$ is semisimple, all its irreducible representations up to conjugacy are labelled by the elements of the set $\Lambda_{f}$ of all Young diagrams with $f$ boxes and the Bratteli diagram of the sequence of Hecke algebras is the same as the one of the group algebras of the symmetric groups.

For $\lambda \in \Lambda_{f}$, let $T_{\lambda}$ denote the set of standard tableaux of shape $\lambda$, and $T_{f}$ the set of all standard tableaux of size $f$. Observe that $t \in T_{\lambda}$ may be identified with a path on Young's lattice (the Bratteli diagram for the sequence of $\mathbf{C} S_{k}$ ) from [1] to $\lambda$, i.e. an increasing sequence of Young diagrams

$$
[1]=\lambda^{(1)} \subset \lambda^{(2)} \subset \ldots \subset \lambda^{(n)}=\lambda,
$$

where $\lambda^{(i)}$ is the diagram which consists of the boxes of $t$ containing the numbers $1,2, \ldots i$.

The irreducible representation $\pi_{\lambda}$ of $H_{f}$ is defined on the vector space $V_{\lambda}$ with (orthonormal) basis labelled by $T_{\lambda}$. We will here use the representations of [W-1] which have the disadvantage that one has to adjoin square roots of certain polynomials. The main reason for doing so is that one obtains unitary matrices for special values of $x$. If one is not interested in unitarity questions, it is better to use Hoefsmit's representations (see $[\mathrm{H}]$ ). His representations can be considered as a $q$ deformation of Young's semiorthogonal representations of the symmetric groups, while ours are deformations of Young's orthogonal representations.

Let $g_{i}(t)$ be the standard tableau obtained by interchanging the numbers $i$ and $i+1$ in the standard tableau $t$ (if $g_{i}(t)$ is not a standard tableau, one sets the corresponding vector equal to 0$)$. Then $\pi_{\lambda}\left(g_{i}\right)$ is defined by

$$
\pi_{\lambda}\left(g_{i}\right) \vec{t}=b_{d} \vec{t}+\frac{\sqrt{\left(1-x^{d+1}\right)\left(1-x^{d-1}\right)}}{1-x^{d}} \vec{g}_{i}(t)
$$

where

$$
d=d(t, i)=c(i+1)-c(i)-r(i+1)+r(i),
$$

with $c(j)$ and $r(j)$ denoting column and row of the box containing the number $j$ and where

$$
b_{d}(x)=\frac{x^{d}(1-x)}{1-x^{d}}
$$

(These differ slightly from corresponding quantities in [W-1] in order to get the usual standard notation for the symmetric group in the case $q=1$; one obtains these coefficients by using the elements $e_{i}$ in [W-1] as spectral projections belonging to the eigenvalue $q$ of $g_{i}$. Then $d$ here is the negative of the $d_{t}$ in [W-1] and $b_{d}=x-(1+x) a_{-d}$, with $a_{d}$ as in [W-1].)

One can check quite directly that

$$
\pi_{\lambda \mid H_{f-1}} \cong \underset{\mu<\lambda}{\bigoplus} \pi_{\mu}
$$

where the sum runs over all Young diagrams which can be obtained by removing exactly one box from $\lambda$. (In particular, the Bratteli diagram for the sequence of $H_{f}(q)$ is the same as that for the sequence of $\left.\mathbf{C}(x) S_{f}.\right)$ 
One can define a family of idempotents $\left\{p_{t}: t \in T_{f}\right\}$ in $H_{f}$ inductively as follows. First, $p_{[1]}=1$. Fix $t \in T_{f}$, and let $r$ be the tableau obtained by removing from $t$ the box containing the number $f$. Then define

$$
p_{t}=\prod_{s} \frac{p_{r} g_{f-1} p_{r}-b_{d(s, f-1)} p_{r}}{b_{d(t, f-1)}-b_{d(s, f-1)}},
$$

where the product is over all $s \in T_{f}$ such that $s \neq t$ but removing from $s$ the box containing the number $f$ also yields the tableau $r$. The family $\left\{p_{t}: t \in T_{n}\right\}$ is a partition of unity consisting of minimal idempotents in $H_{n}$ (see [W-1, Corollary 2.3]).

Observe that the construction of the representations and idempotents also works for the complex algebras $H_{f}(q)$, as long as $q$ is not a primitive $l^{\text {th }}$ root of unity with $l=2,3, \ldots f$. This follows from the fact that all the rational functions occurring above only have poles at such roots of unity.

If $q$ is a primitive $l^{\text {th }}$ root of unity one can still define semisimple representations of $H_{f}(q)$ for special diagrams:

(a) The diagram $\lambda$ will be called $l$ regular if its largest hook contains less than $l$ boxes or equivalently, if

$$
h(1,1)=\lambda_{1}+\tilde{\lambda}_{1}-1<l .
$$

A standard tableau is called $l$ regular if it belongs to an $l$ regular diagram. Note that we have for any $l$ regular tableau $t$ that $d(t, i)<l-1$ for $1 \leqq i<f$.

(b) Let $k \in \mathbf{N}$ with $1 \leqq k<l$. The diagram $\lambda$ is called a $(k, l)$ diagram if it has $k$ rows at the most and if

$$
\lambda_{1}-\lambda_{k} \leqq l-k \text {. }
$$

The set of diagrams satisfying the conditions above is denoted by $\Lambda^{(k, l)}$.

Observe that any $l$ regular diagram with $k$ rows is a $(k, l)$ diagram.

Proposition 2.1. Let $q$ be a primitive $l^{\text {th }}$ root of unity.

(a) Let $\lambda \in \Lambda_{f}$ be l regular and let $z_{\lambda}$ be the central idempotent in $H_{f}$ corresponding to $\pi_{\lambda}\left(H_{f}\right)$. Then $z_{\lambda}(q)$ is well-defined and $z_{\lambda}(q) H_{f}(q)$ is semisimple (and isomorphic to $\left.\pi_{\lambda}\left(H_{f}(q)\right)\right)$. In particular, $H_{f}(q)$ has a direct summand $H_{f}^{r}(q)$ whose simple components are labelled by l-regular diagrams.

(b) Let $\varrho$ be a representation of $H_{f+1}(q)$ whose kernel contains $H_{f+1}^{r}(q)$. Then its restriction to $H_{f}(q)$ does not contain any subrepresentation belonging to an $l-1$ regular diagram.

(c) Let $\lambda \in \Lambda_{f}^{(k, l)}$. There exists a semisimple representation $\pi_{\lambda}^{(k, l)}$ of $H_{f}(q)$ such that its restriction to $H_{f-1}(q)$ decomposes as in (2.3) except that now only those $\mu$ occur on the right-hand side which are also $(k, l)$ diagrams. If $q=e^{2 \pi i / l}, \pi_{\lambda}^{(k, l)}\left(g_{i}\right)$ is a unitary matrix for $i=1,2, \ldots|\lambda|-1$.

Proof. (a) We proceed by induction on $f$. If $t_{0}$ is $l$ regular, then so is $r=t_{0}^{\prime}$. By induction assumption, $p_{r}$ is a well-defined minimal idempotent in $H_{f-1}(q)$ belonging to an $l$ regular diagram with $f-1$ boxes. Observe that the formula for $p_{t_{0}}$ makes sense only if $b_{d(t, f-1)} \neq b_{d(s, f-1)}$ for all tableaux $t$ such that $t^{\prime}=r$. By [W-1], Lemma (2.1), this is the case if $\left|d_{t}-d_{t_{0}}\right|<l$. This difference is just the length of the hook through the boxes containing $f$ in $t$ and $t_{0}$ minus 1 . This is less than $l$ because $r$ with these 2 boxes belongs to an $l+1$ diagram. Hence $p_{t_{0}}(q)$ is well-defined. The semisimplicity of $\pi_{\lambda}$ is shown as in [W-1] Corollary 2.5. 
(b) Let for the generic Hecke algebra $H_{r}$ the idempotent $z_{r}^{(s)}$ be the sum of all central idempotents $z_{\lambda}$ belonging to $s$-regular diagrams $\lambda$ with $r$ boxes. Obviously, a diagram obtained from an $l-1$ regular diagram by adding a box has to be $l$ regular. Hence it follows from (2.3) that $z_{f-1}^{(l-1)} z_{f}^{(l)}=z_{f-1}^{(l-1)}$. By (a) these idempotents are also well-defined for $x=q$ for which the same algebraic relations hold. So if $z_{l}^{(f)}(q)$ is in the kernel of $\varrho$, then also $z_{f-1}^{(l-1)}(q)$.

(c) follows from [W-1, Corollary 2.5 and Proposition 2.10].

It is possible to characterize also the maximal semisimple quotients of $H_{l}(q)$ and $H_{l+1}(q)$ for $q$ a primitive $l^{\text {th }}$ root of unity. Most (presumably all) of these results are well-known to experts of Hecke algebras (see e.g. [DJ] and [Y]).

Proposition 2.2. Let $q$ be a primitive $l^{\text {th }}$ root of unity and let $\bar{H}_{f}(q)$ be the maximum semisimple quotient of $H_{f}(q)$. Then we have the following facts:

(a) All diagrams with l boxes are l regular except the hook diagrams (including $[l]$ and $\left[1^{l}\right]$ and the maximal semisimple quotient $\bar{H}_{l}(q)$ is a direct sum of $H_{l}^{r}(q)$ and $H_{l}^{\prime}(q)$, where $H_{l}^{\prime}(q)$ is the direct sum of the $(k, l)$ representations belonging to hook diagrams of the form $\left[l-k+1,1^{k-1}\right], 1 \leqq k<l$, i.e.

$$
H_{l}^{\prime}(q)=\bigoplus_{k=1}^{l-1} \pi_{\left[l-k+1,1^{k-1}\right]}^{(k, l)}\left(H_{l}(q)\right)
$$

In this case the restriction of an irreducible representation in $H_{l}^{\prime}(q)$ to $H_{l-1}(q)$ remains irreducible. In particular, if the restriction of an irreducible representation of $H_{l}(q)$ to $H_{l-1}(q)$ only contains simple components labelled by hook diagrams, it has to be in $H_{l}^{\prime}(q)$.

(b) One can write $\bar{H}_{l+1}(q)$ as a direct sum of $H_{l+1}^{r}(q)$ and $H_{l+1}^{\prime}(q)$, where

$$
H_{l+1}^{\prime}(q) \cong \bigoplus_{k=1}^{l} \pi_{\left[l+1-k, 1^{k}\right]}\left(H_{l+1}(q)\right) \oplus \bigoplus_{k=2}^{l-1} \pi_{\left[l-k+1,2,1^{k-2}\right]}^{(k, l)}\left(H_{l+1}(q)\right) \text {. }
$$

It should be noted that the representation in [W-1] for hook diagrams with $l+1$ boxes are not well-defined if $q$ is a primitive $l^{\text {th }}$ root of unity (even though the corresponding central idempotent is). In this case one can, for instance, take the representation in $[\mathrm{KL}]$ on the $W$ graph corresponding to such hook diagrams (see [GMcL]). Observe that by [W-1, Sect. 2] one has

$$
\pi_{\left[l-k+1,2,1^{k-2}\right] \mid H_{l}(q)}^{(k, l)}=\pi_{\left[l-k+1,1^{k-1}\right]}^{(k, l)} \oplus \pi_{\left[l-k, 2,1^{k-2}\right]} .
$$

(c) The restriction of the representations corresponding to hook diagrams in (b) are not semisimple except for the trivial representations belonging to $[l+1]$ and $\left[1^{l+1}\right]$. More generally, if $\varrho$ is a representation of $H_{l+1}(q)$ whose restriction to $H_{l}(q)$ is semisimple and does not contain any representation labelled by a diagram of the form $\left[l-k, 2,1^{k-2}\right]$ for $k=2,3, \ldots l-2$, then $\varrho$ is a quotient of $H_{l+1}^{r}(q)$.

Proof. The irreducible components of $\bar{H}_{f}(q)$ can be labelled by diagrams which are $l$-regular in the sense of [DJ]. By this one means diagrams for which the number of boxes in 2 consecutive columns does not differ by more than $l-1$ boxes (see [DJ]). It is easy to check that our expressions for $\bar{H}_{l}(q)$ respectively $\bar{H}_{l+1}(q)$ contain the right number of simple components. It remains to show that these are mutually nonisomorphic semisimple representations. For $l$ regular and $(k, l)$ representations it can be checked easily that already the restrictions to $H_{l-1}(q)$ respectively $H_{l}(q)$ of representations belonging to different diagrams are nonisomorphic using (2.3) (see [W-1], Sect. 2). 
To prove the theorem, it suffices to show for each hook diagram $\lambda$ with $l+1$ boxes that the central idempotent $z_{\lambda}(q)$ is well-defined, that the corresponding representation is irreducible and that its restriction to $H_{l}(q)$ is not semisimple. To do so, one first shows inductively that while the idempotents $p_{t}, t \in T_{\lambda}$ are in general not well-defined at $q$ a root of unity, the idempotent $p_{t}+p_{g_{l}(t)}$ is so (one obtains a cancellation of a singularity). Here we also use the convention that $p_{g_{i}(t)}=0$ if $g_{i}(t)$ is not a standard tableau. Hence also $z_{\lambda}(q)=\sum p_{t}(q)$ is well-defined, where the summation goes over all tableaux of shape $\lambda$.

To show that the corresponding representation is irreducible let $e_{l}$ be a spectral idempotent of $g_{l}$. Then one obtains a partition of unity, in $\pi_{\lambda}\left(H_{l+1}\right)$, of mutually orthogonal minimal idempotents of the form $\left\{\left(p_{t}+p_{g_{l}(t)}\right) e_{l},\left(p_{t}+p_{g_{l}(t)}\right)\left(1-e_{l}\right)\right.$, $\left.t \in T_{\lambda}\right\}$ which is also well-defined for our special choice of $q$. One now obtains matrix units applying Proposition 1.3 to any pair of these idempotents.

To show (c), let $\varrho$ be an irreducible representation of $H_{l+1}(q)$ corresponding to a hook diagram $\lambda$. As $H_{l-1}(q)$ is semisimple, $\varrho\left(H_{l-1}(q)\right)$ decomposes (as in the semisimple case) as a direct sum of representations labelled by all subdiagrams of $\lambda$ with $l-1$ boxes (all of which are also hooks).

Hence, if $\varrho\left(H_{l}(q)\right)$ were semisimple, it would have the same central idempotents as $\varrho\left(H_{l-1}(q)\right)$ by (a). But as they are elements of $\varrho\left(H_{l-1}(q)\right)$, they would also commute with $\varrho\left(g_{l}\right)$, i.e. they would be central which would contradict the irreducibility of $\varrho$. The second statement follows from this and the other statements in (b).

We still need another algebra, the so-called Brauer algebra (see [Br] and [W-2]). As before with the Hecke algebras, Brauer's algebras $D_{f}$ will first be defined over $\mathbf{C}(x)$. For $f=0, D_{0}=\mathbf{C}(x)$. For $f>0$, a linear basis of the $\mathbf{C}(x)$ algebra $D_{f}$ is given by graphs with $f$ edges and $2 f$ vertices, arranged in 2 lines of $f$ vertices each. In these graphs each edge belongs to exactly 2 vertices and each vertex belongs to exactly one edge. So an example for a graph in $D_{4}$ would be

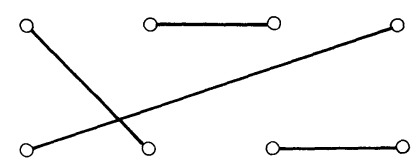

Fig. 2

It is easy to see that there are $2 f-1$ possibilities to join the first vertex with another one, then $2 f-3$ possibilities for the next one and so on. So the dimension of $D_{f}$ is $135 \ldots(2 f-1)$. To define the multiplication in $D_{f}$, it is enough to define the product $a b$ for 2 graphs $a$ and $b$. This is done similarly as with braids by the following rule.

(a) Draw $b$ below $a$.

(b) Connect the $i^{\text {th }}$ upper vertex of $b$ with the lower $i^{\text {th }}$ vertex of $a$.

(c) Let $d$ be the number of cycles in the new graph obtained in (b) and let $c$ be this graph without the cycles. Then $a b=x^{d} c$.

We will call an edge horizontal if it joins 2 vertices in the same row. Note that there are as many horizontal edges in the upper row as there are in the lower one. Whenever a graph $p$ has no horizontal edges, it can be regarded as a permutation $\pi$ 
connecting the $i^{\text {th }}$ lower vertex to the $\pi(i)^{\text {th }}$ upper vertex. So, obviously, $D_{f}$ contains $\mathbf{C}(x) S_{f}$ as a subalgebra. Let $g_{i}$ be the graph which has only vertical edges except for the $i^{\text {th }}$ and $(i+1)^{\text {st }}$ one, which are crossing (the graph corresponding to the picture of $\sigma_{i}$ in Fig. 4). It is easy to check that $g_{i}$ corresponds to the transposition $(i, i+1)$. We will also need the elements $e_{i}$ given by graphs, in which the $i^{\text {th }}$ and $(i+1)^{\text {th }}$ vertices are connected by horizontal edges and all other edges are vertical. We also remark that for any graph $b \in D_{f}$ and a permutation graph $p$ the graph $b p$ is obtained by permuting the vertices of the lower row of $b$ by $\pi^{-1}$ and $p b$ is the graph obtained by permuting the vertices of the upper row of $b$ by $\pi$.

We finally remark that $D_{f}$ can be identified with the subalgebra of $D_{f+1}$ spanned linearly by all graphs with a vertical edge on their right-hand sides.

The $\mathbf{C}$ algebra $D_{f}(q)$ has a linear basis labeled by the same graphs. The multiplication is defined as in $D_{f}$ except that every occurrence of $x$ is replaced by $q$. As we will have to divide by our parameter $q$ later, we will always assume $q \neq 0$ even though $D_{f}(0)$ is well-defined.

We have the following results about the structure of Brauer's algebras (see [Br, Wy, W-2]).

Theorem 2.3. (a) $D_{f} \cong I_{f} \oplus \mathbf{C} S_{f}$, where $I_{f}$ is isomorphic to Jones' basic construction for $D_{f-2} \subset D_{f-1}$. In particular, $D_{f}$ is semisimple.

(b) The simple components of $D_{f}$ are labelled by the set $\Gamma_{f}$ consisting of all Young diagrams with $k$ boxes where $0 \leqq k \leqq f$ and $f-k$ is even.

(c) If $V_{f, \lambda}$ is a simple $D_{f, \lambda}$ module, it decomposes as a $D_{f-1}$ module into a direct sum

$$
V_{f, \lambda}=\oplus_{\mu \leftrightarrow \lambda} V_{f-1, \mu},
$$

where $V_{f-1, \mu}$ is a simple $D_{f-1, \mu}$ module and $\mu$ runs through all diagrams obtained by removing or (if $\lambda$ contains less than $f$ boxes) adding a box to $\lambda$.

(d) $D_{f}(q)$ is semisimple except if $q$ is an integer with absolute value at most $f$.

As a consequence of this theorem, one obtains the structure of semisimple $D_{f}$ 's inductively from the following Bratteli diagram:

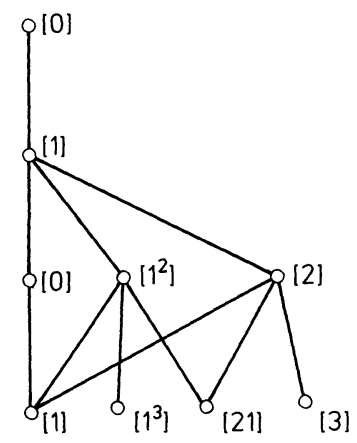

Fig. 3 


\section{The Algebras $C_{f}$ and $C_{f}(r, q)$}

We shall consider algebras $C_{f}, f \in \mathbf{N}$ over the field $\mathbf{C}(r, q)$ of rational functions in 2 variables $r$ and $q$ as well as their complex versions $C_{f}(r, q), f \in \mathbf{N}$ where the complex parameters are denoted by the same symbols. This should not cause any confusion after the discussion in Sect. 1.

These algebras were originally derived from the Kauffman link invariant (see [BW, M-1]). While our present work could be done completely without using this invariant, it still seems worthwhile recalling this connection to motivate the algebraic relations which will be used later. We shall also use this opportunity to simplify parts of the exposition in [BW].

Let us first recall a few basic facts of knot theory (for some detailed expositions see for instance [Jo-2, K, Bi, BW, W-3]). A link is a smooth embedding of one or several copies of the circle $S^{1}$ (with orientations) into $\mathbf{R}^{3}$. Two links are considered to be equivalent if there exists a homeomorphism from $\mathbf{R}^{3}$ onto itself which maps one link onto the other preserving the chosen orientations. It follows from a theorem by Alexander that any link can be obtained as the closure $(\hat{\beta}, f)$ of a braid $\beta \in B_{f}$ (see e.g. [BW], Fig. 1 and 3 for an example or Fig. 5 for the corresponding 2 dimensional version).

The Kauffman invariant $K$ is defined by a renormalization (i.e. by multiplication by a power of $r$; see discussion at the end of this section) of an invariant $\widetilde{K}$ of regular isotopy. By this one means an invariant of (unoriented) link diagrams (i.e. projections of links into a plane without triple points, where at each crossing point it is indicated which string goes over the other one). Two such link projections are considered to be equivalent with respect to regular isotopy if one of them can be obtained from the other one only by moves within the plane (i.e. without the third Reidemeister move; see [K or BW] for details). The invariant $\widetilde{K}$, which depends on 2 parameters $r$ and $q$, is defined inductively by the following relations (where we use a slightly different parametrization than Kauffman)

$$
\begin{aligned}
& \tilde{K}_{\bigcirc}=1, \\
& \widetilde{K}_{1}=r^{-1} \widetilde{K}_{\varphi}=r \widetilde{K}_{p}, \\
& \tilde{K}_{\lambda^{-}}-\tilde{K}_{\text {以 }}=\left(q-q^{-1}\right)\left(\tilde{K}_{\|}-\tilde{K}_{=}\right) \text {. }
\end{aligned}
$$

Here, the last 2 lines relate the invariants of link diagrams which are identical everywhere except in a small square where they look like the pictures indicated. It follows from Kauffman's work that (K1) - (K3) determine a well-defined invariant of link diagrams. The corresponding link invariant is obtained by multiplying this invariant by $r$ taken to the power of the number of positive crossings minus the negative crossings (the first crossing in (K3) would be positive, the second one negative).

In [W-3] we described a general procedure how to obtain representations of braid groups from link invariants satisfying certain properties. This could be applied to Kauffman's invariant; due to its definition as basically an invariant of regular isotopy, it seems more naturally to give the corresponding 2-dimensional version of our procedure here.

We first do that for the braid groups (the reason being that closures of different projections of the same braid may be different with respect to regular isotopy). So we represent the generators $\sigma_{i}$ and $\sigma_{i}^{-1}$ by the projections in Fig. 4, i.e. by $f$ strings 
of which $f-2$ are going straight down and the $i^{\text {th }}$ and $(i+1)^{\text {st }}$ are as indicated. A general braid $\beta=\prod \sigma_{m_{j}}^{n_{j}}$ is now given by the equivalence class modulo regular isotopy of the picture obtained by the corresponding concatenation of the picture of the generators. Because of this definition, the closure $(\hat{\beta}, f)$ of the braid $\beta$, obtained as usual by joining the $i^{\text {th }}$ upper point with the $i^{\text {th }}$ lower point by a line without any loops, represents a well-defined equivalence class of regular isotopy. So, with

$$
x=\frac{r-r^{-1}}{q-q^{-1}}+1
$$

one obtains the well-defined functional $\operatorname{tr}$ on $\mathbf{C}(r, q) B_{\infty}$ by (for an example of the closure $(\hat{\beta}, f)$ see Fig. 5)

$$
\operatorname{tr}(\beta)=x^{1-f} \tilde{K}\left((\hat{\beta}, f) \text { for } \beta \in B_{f} .\right.
$$

Then it is easy to check by pictures, using the definition of $\widetilde{K}$ that

(1) $\operatorname{tr}(\beta \gamma)=\operatorname{tr}(\gamma \beta)$ for $\beta, \gamma \in B_{f}$,

(2) $\operatorname{tr}\left(\beta \sigma_{f}^{ \pm 1}\right)=\operatorname{tr}(\beta) \operatorname{tr}\left(\sigma_{f}^{ \pm 1}\right)$ and

(3) $\operatorname{tr}\left(\sigma_{i}^{ \pm 1}\right)=r^{ \pm 1} / x$.

Functionals on $B_{\infty}$ which satisfy (1) and (2) are usually called Markov traces. If they also satisfy $\operatorname{tr}\left(\sigma_{i}\right)=\operatorname{tr}\left(\sigma_{i}^{-1}\right)$, they are called normalized (this is often already assumed for Markov traces). If this is not the case (as for instance in our case), it can be easily remedied by rescaling (see the end of this section).

Let now $\pi_{\mathrm{tr}}$ be the GNS construction with respect to tr and let $g_{i}=\pi_{\mathrm{tr}}\left(\sigma_{i}\right)$ for $i=1,2, \ldots$ The similarity between the crossings on the left-hand side of $(\mathrm{K} 3)$ and the standard generators of the braid group suggests the definition of elements $e_{i} \in \pi_{\mathrm{tr}}\left(\mathbf{C}(r, q) B_{\infty}\right)$ by

$$
e_{i}=1-\frac{1}{q-q^{-1}}\left(g_{i}-g_{i}^{-1}\right)
$$

We will show that the algebra $C_{f}$ can be described by adding to our 2-dimensional description of the braid group $B_{f}$ additional generators $\varepsilon_{i}$ as indicated below. This obviously suggests a close relationship with Brauer's centralizer algebras. The main difficulty is to get rid of cycles which are no longer connected to the upper or lower end. For this see [MW].
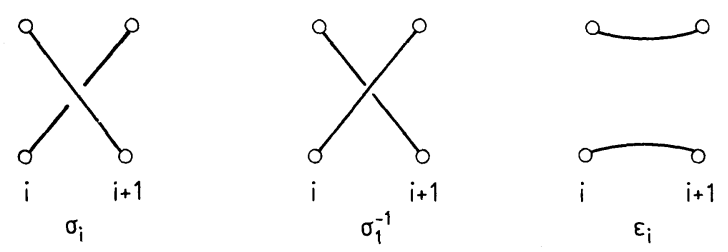

Fig. 4

We shall show that the element $e_{i}$, defined above, satisfy similar relations as the corresponding graphs of Brauer's algebras. 
Lemma 3.1. (i) Let $\beta \in B_{f}$ be a braid. Then $\operatorname{tr}\left(e_{i} \pi_{\mathrm{tr}}(\beta)\right)$ is equal to $x^{1-f} \tilde{K}\left(\widehat{\varepsilon_{i} \beta}, f\right)$. (ii) Similarly, if $m$ is a product of $g_{i}{ }^{\prime} s, g_{i}^{-1}, s$ and $e_{i}{ }^{\prime} s, \operatorname{tr}\left(m \pi_{\mathrm{tr}}(\beta)\right)$ is equal to $x^{1-f} \widetilde{K}(\hat{\beta}, f)$, where $\mu$ is obtained from the corresponding concatenation of $\sigma_{i}{ }^{\prime}$, $\sigma_{i}^{-1}$ 's and $\varepsilon_{i}$ 's (see also the proof of (iii) for an example).

(iii) One has the following relations between $g_{i}$ 's and $e_{i}$ 's:

(R1) $e_{i} g_{i}=r^{-1} e_{i}$

(R2) $e_{i} g_{i}^{ \pm 1} e_{i}=r^{ \pm 1} e_{i}$.

Proof. By (D) we can write for $\operatorname{tr}\left(e_{i} \pi_{\mathrm{tr}}(\beta)\right)$ the linear combination $\operatorname{tr}(\beta)-\left(\operatorname{tr}\left(\sigma_{i} \beta\right)\right.$ $\left.-\operatorname{tr}\left(\sigma_{i}^{-1} \beta\right)\right) /\left(q-q^{-1}\right)$. By definition of tr below (3.1) this linear combination is equal to a linear combination of invariants of link projections which only differ at one crossing. The claim follows now from (K3) of the definition of the invariant $\widetilde{K}$.

(ii) Can be shown by induction on the number of $e_{i}$ 's in $m$ using (i) (i.e. expand one of the $e_{i}$ 's as a linear combination of $g_{i}, g_{i}^{-1}$ and 1 and apply induction assumption).

Observe that $\mathrm{tr}$ is nondegenerate on $\pi_{\mathrm{tr}}\left(\mathbf{C} B_{f}\right)$. So to prove (R2), it is enough to show that

$$
\operatorname{tr}\left(e_{i} g_{i-1}^{ \pm 1} e_{i} \pi_{\mathrm{tr}}(\beta)\right)=r^{ \pm 1} \operatorname{tr}\left(e_{i} \pi_{\mathrm{tr}}(\beta)\right) \text { for all } \beta \in B_{\infty},
$$

as the $\pi_{\mathrm{tr}}(\beta), \beta \in B_{\infty}$ obviously span $\pi_{\mathrm{tr}}\left(\mathbf{C} B_{\infty}\right)$ linearly. By (i) this is equivalent to showing that

$$
\widetilde{K}\left(\widehat{\varepsilon_{i} \sigma_{i-1}^{ \pm 1} \varepsilon_{i} \beta}, f\right)=r^{ \pm 1} \widetilde{K}\left(\widehat{\varepsilon_{i} \beta}, f\right) \text {. }
$$

This follows immediately from (K2) and the following picture (where we have assumed $f=3$ and $\beta=1$ to simplify the drawing; the argument is exactly the same for a more complicated picture)
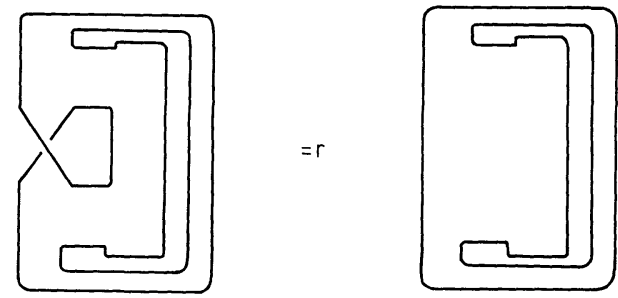

Fig. 5

Relation (R1) is shown in a similar fashion.

The previous lemma provides the motivation for defining complex algebras by generators satisfying the braid relations and the relations (R1) and (R2) (a different set of relations for which the algebra is also well-defined at $(r, q)=(1,1)$ is given after Proposition 3.2). More precisely, the complex algebra $C_{f}(r, q)$ is given by generators $g_{1}, g_{2}, \ldots g_{f-1}$, which are assumed to be invertible, and relations

(B1) $g_{i} g_{i+1} g_{i}=g_{i+1} g_{i} g_{i+1}$,

(B2) $g_{i} g_{j}=g_{j} g_{i}$ if $|i-j| \geqq 2$,

(R1) $e_{i} g_{i}=r^{-1} e_{i}$,

(R2) $e_{i} g_{i-1}^{ \pm 1} e_{i}=r^{ \pm 1} e_{i}$, 
where $e_{i}$ is defined by the equation

$$
\left(q-q^{-1}\right)\left(1-e_{i}\right)=g_{i}-g_{i}^{-1} \text {. }
$$

Multiplying (D) by $e_{i}$ and solving for $e_{i}^{2}$ yields

$$
e_{i}^{2}=x e_{i}
$$

with $x$ as in (3.1). This together with (R1) shows that $e_{i}$ is a multiple of the characteristic idempotent $p_{i}$ belonging to the characteristic value $r^{-1}$ of $g_{i}$. Multiplying the equation (D) by $\left(1-p_{i}\right)$ then shows that the only other possible characteristic values of $g_{i}$ are $q$ and $-q^{-1}$. Hence $g_{i}$ also satisfies

$(\mathrm{R} 1)^{\prime}\left(g_{i}-r^{-1}\right)\left(g_{i}+q^{-1}\right)\left(g_{i}-q\right)=0$.

In the rest of this section, we construct the functional tr on this algebra by elementary methods (see (3.3)) without assuming the existence of the Kauffman polynomial. This will then be used to determine the complete decomposition of $C_{f}$ into simple matrix rings (see Theorem 3.6). We will need the following relations which can either be derived from the defining relations (see below) or by drawing pictures of link diagrams (see e.g. [BW, Fig. 6])

(1) $g_{i \pm 1}^{-1} g_{i} g_{i \pm 1}=g_{i} g_{i \pm 1} g_{i}^{-1}$,

(2) $e_{i \pm 1} g_{i} g_{i \pm 1}=g_{i} g_{i \pm 1} e_{i}$

(3) $g_{i} e_{i \pm 1} g_{i}^{-1}=g_{i \pm 1}^{-1} e_{i} g_{i \pm 1}$,

(4) $e_{i} g_{i+1}^{ \pm 1} e_{i}=r^{ \pm 1} e_{i}$,

(5) $g_{i} e_{i \pm 1} e_{i}=g_{i \pm 1}^{-1} e_{i}$,

(6) $e_{i} e_{j}=e_{j} e_{i}$ if $|i-j| \geqq 2$,

(7) $e_{i}^{2}=x e_{i}$,

(8) $e_{i} e_{i \pm 1} e_{i}=e_{i}$,

(9) $e_{i-1} g_{i} g_{i-1}=e_{i-1} e_{i}$,

(10) $g_{i}^{2}=\left(q-q^{-1}\right)\left(g_{i}+r^{-1} e_{i}\right)+1$.

Most of these relations follow immediately from the defining relations and the previous ones. We restrict ourselves to proving (5). Applying (2), (R2) and (4), we obtain

$$
g_{i} e_{i \pm 1} e_{i}=g_{i \pm 1}^{-1}\left(g_{i \pm 1} g_{i} e_{i \pm 1}\right) e_{i}=g_{i \pm 1}^{-1}\left(e_{i} g_{i \pm 1} g_{i}\right) e_{i}=r^{-1} g_{i \pm 1}^{-1} e_{i} g_{i \pm 1} e_{i}=g_{i \pm 1}^{-1} e_{i} \text {. }
$$

Relations (7), (8) and (10) are shown by multiplying (D) by $e_{i}$ from the left and from both sides and by $g_{i}$ respectively. (9) follows from

$$
e_{i-1} g_{i} g_{i-1}=e_{i-1}\left(e_{i} e_{i-1} g_{i}\right) g_{i-1}=e_{i-1} e_{i} g_{i-1}^{-1} g_{i-1}=e_{i-1} e_{i} \text {. }
$$

The following proposition contains easy consequences of these relations (see also [BW, Sect. 3]):

Proposition 3.2. (a) Any element of $C_{f+1}$ can be written as a linear combination of elements of the form $a \chi b$ with $\chi \in\left\{1, g_{f}, e_{f}\right\}$ and $a, b \in C_{f}$. In particular, it follows from this by induction on $f$ that $C_{f+1}$ is finite dimensional. 
(b) Let $I_{f+1}$ be the ideal in $C_{f+1}$ generated by $e_{f}$. Then each element in $I_{f+1}$ can be written as a linear combination of elements of the form ae $b$ with $a, b \in C_{f}$. In particular, $I_{f+1} \subset\left\langle C_{f}, e_{f}\right\rangle$. Moreover, the quotient is isomorphic to the Hecke algebra $H_{f+1}\left(q^{2}\right)$.

(c) The structure of $C_{f}$ remains unchanged under the following changes of parameters:

$$
\begin{aligned}
& (r, q) \mapsto\left(r,-q^{-1}\right), \\
& (r, q) \mapsto(-r,-q), \\
& (r, q) \mapsto\left(r^{-1}, q^{-1}\right) .
\end{aligned}
$$

In particular, if $|r|=|q|=1$, one can assume without restriction of generality that

$$
q=e^{i t}, \quad 0 \leqq t \leqq \pi / 2 \quad \text { and } \quad r=e^{i s}, \quad-\pi / 2 \leqq s \leqq \pi / 2 .
$$

Similarly, if both $r$ and $q$ are real, one can assume

$$
q \geqq 1 \text { and } r>0 \text {. }
$$

Proof. (a) and (b) follow from the relations above (see also [BW]). For (c), let $g_{i}\left(r^{-1}, q^{-1}\right), i=1,2, \ldots f-1$ be the standard generators of $C_{f}\left(r^{-1}, q^{-1}\right)$ and let $\Phi: g_{i}\left(r^{-1}, q^{-1}\right) \mapsto g_{i}^{-1} \in C_{f}(r, q)$. It is easy to show that $\Phi$ preserves the relations and can be extended to a homomorphism from $C_{f}(r, q)$ onto $C_{f}\left(r^{-1}, q^{-1}\right)$ mapping $e_{i}\left(-r, q^{-1}\right)$ to $e_{i}$. In the same way, we can construct the inverse map to show that $\Phi$ is an isomorphism.

It can be shown in the same fashion that the maps $g_{i}(-r,-q) \mapsto-g_{i}$ and $g_{i}\left(r,-q^{-1}\right) \mapsto g_{i}$ can be extended to isomorphisms between $C_{f}(-r,-q)$ and $C_{f}(r, q)$ and between $C_{f}\left(r,-q^{-1}\right)$ and $C_{f}(r, q)$, respectively.

To show the connection between the $C_{f}$ 's and Brauer's centralizer algebras, one needs a different choice of parameters, which results into a so-called "blowing-up" of the point $(1,1)$ :

The quantity $x$, defined in (3.1) will be introduced as a new variable replacing $q$. Then the algebra $\widehat{C}_{f}(r, x)$ is defined by the same generators and relations as above where the quantity $q-q^{-1}$ is replaced by $\left(r-r^{-1}\right) /(x-1)$. This does not affect relations (B1), (B2) and (R2) while (R1) changes to

$$
\left(g_{i}-r^{-1}\right)\left(g_{i}^{2}-\frac{r-r^{-1}}{x-1} g_{i}-1\right)=0 \text {, }
$$

and (D) changes to

$$
\frac{r-r^{-1}}{x-1}\left(1-e_{i}\right)=g_{i}-g_{i}^{-1}
$$

As one can not solve for $e_{i}$ if $r= \pm 1$, one introduces $e_{1}, e_{2} \ldots e_{n-1}$ as additional generators (actually, $e_{1}$ alone would be enough by (3)). Then one also introduces relations (1)-(10) as additional defining relations. The following lemma will be useful for getting a lower bound for the dimension of $C_{f}$ and for defining the structure trace tr on it.

Lemma 3.3. (a) The map $\Psi$ which maps the elements $g_{i}$ and $e_{i}$ to the graphs of the Brauer algebra $D_{f}$ which are denoted by the same symbol (which correspond to the pictures for $\sigma_{i}$ and $\varepsilon_{i}$ in Fig. 4) extends to a homomorphism from $\hat{C}_{f}(1, x)$ onto $D_{f}$. 
(b) Assume that $\Psi$ is a $\mathbf{C}(x)$ algebra isomorphism between $\hat{C}_{f}(1, x)$ and $D_{f}$ and that the $\mathbf{C}(x)$-dimension of $D_{f}$ coincides with the $\mathbf{C}(x, r)$-dimension of $\hat{C}_{f}$. Then

$$
C_{f} e_{f+1} \cong C_{f} .
$$

Proof. It follows immediately from the defining relations that the map $\Phi: a \in C_{f}$ $\mapsto(1 / x) e_{f+1} a \in C_{f+2}$ is a homomorphism. By assumption, there exists a basis $B$ of $\hat{C}_{f}$ which specializes for $r=1$ to the basis graphs of $D_{f}$. But for the Brauer algebras, the map $\Phi$ can be interpreted as just adding two horizontal edges on the right to each graph. Obviously, the images of the basis graphs are also linearly independent. But then so are the images of our chosen basis of $C_{f}$ (or $\hat{C}_{f}$ respectively) by Proposition 1.3 (with $p=1$ ).

By Proposition 3.2, (a), any element of $C_{f+1}$ can be written as a linear combination of elements of the form $a \chi b$ with $\chi \in\left\{1, g_{f}, e_{f}\right\}$ and $a, b \in C_{f}$. Now observe that by (R2) and (7) we have $e_{f+1} a \chi b e_{f+1}=\alpha a b e_{f+1}$ with $\alpha \in\{1, x\}$. Hence, if the hypotheses of the previous lemma are satisfied, it follows from this and Proposition 3.2 that there exists for all elements $a \in C_{f}$ a unique element $\varepsilon_{f}(a) \in C_{f}$ such that

$$
e_{f+1} a e_{f+1}=x \varepsilon_{f}(a) e_{f+1} .
$$

As in [W-2] it is easy to check that the map $a \mapsto \varepsilon_{f}(a)$ is linear. Moreover, as $e_{f+1}$ commutes with $C_{f}$, it follows directly from the definition (3.2) that $\varepsilon_{f}$ has the socalled bimodule property, i.e. $\varepsilon_{f}\left(b_{1} a b_{2}\right)=b_{1} \varepsilon_{f}(a) b_{2}$ for $b_{1}, b_{2} \in C_{f}$ and $a \in C_{f+1}$. In particular, it follows from this that the restriction of $\varepsilon_{f}$ to $C_{f}$ is the identity map. This is used to define a functional $\operatorname{tr}$ on $C_{f}$ inductively by $\operatorname{tr}(1)=1$ and

$$
\operatorname{tr}(a)=\operatorname{tr}\left(\varepsilon_{f}(a)\right) \text { for } a \in C_{f+1} .
$$

We are going to show that this functional tr agrees with the one derived from Kauffman's invariant. Assuming the existence of that functional, this would already follow from part (d) of the following lemma. Parts (e) and (f) are only needed to prove the existence of tr without using Kauffman's work.

Lemma 3.4. Assume the hypotheses of Lemma 3.3.

(a) If $\operatorname{tr}$ is well-defined on $C_{f}$, it extends to a well-defined functional on $C_{f+1}$.

(b) $\operatorname{tr}\left(e_{i}\right)=1 / x$ and $\operatorname{tr}\left(g_{i}^{ \pm 1}\right)=r^{ \pm 1} / x$ for $i=1,2, \ldots f-1$, where $x=\left(r-r^{-1}\right) /\left(q-q^{-1}\right)$ +1 .

(d) $\operatorname{tr}(a \chi b)=\operatorname{tr}(\chi) \operatorname{tr}(a b)$ and $\varepsilon_{f}(a \chi b)=\operatorname{tr}(\chi) a b$ for $\chi \in\left\{e_{f}, g_{f}\right\}$ and $a, b \in C_{f}$. In particular, any functional $\phi$ on $C_{\infty}$ which satisfies the first property of this statement for all $f \in \mathbf{N}$ has to coincide with tr.

(e) If $b \in C_{f}$ one has

$$
\varepsilon_{f}\left(g_{f}^{-1} b g_{f}\right)=\varepsilon_{f}\left(g_{f} b g_{f}^{-1}\right)=\varepsilon_{f}\left(e_{f} b e_{f}\right)=\varepsilon_{f-1}(b) .
$$

(f) If $\operatorname{tr}$ is a trace on $C_{f}$ and if $I_{f+1}$ is semisimple, then $\operatorname{tr}$ is also a trace on $C_{f+1}$. Proof. (a) follows immediately from the inductive definition and from $\varepsilon_{f}(a)=a$ for all $a \in C_{f}$. (b) follows from (R2) and (8).

To prove (e), first recall that any element of $b \in C_{f}$ can be written as a linear combination of elements of the form $a \chi b$ with $\chi \in\left\{1, g_{f-1}, e_{f-1}\right\}$ and $a, b \in C_{f-1}$. Hence, as $\varepsilon_{f}(a \chi b)=\operatorname{tr}(\chi) a b$ by (d), the claim would follow from

$$
e_{f+1}\left(g_{f}^{-1} \chi g_{f}\right) e_{f+1}=e_{f+1}\left(g_{f} \chi g_{f}^{-1}\right) e_{f+1}=e_{f+1}\left(e_{f} \chi e_{f}\right) e_{f+1}=x \operatorname{tr}(\chi) e_{f+1} \text {. }
$$


It is easy to check this for $\chi=1$. Let $\chi=e_{f-1}$. Then, using relation (3), one has $e_{f+1} g_{f}^{-1} e_{f-1} g_{f} e_{f+1}=e_{f+1} g_{f-1} e_{f} g_{f-1}^{-1} e_{f+1}=g_{f-1} e_{f+1} e_{f} e_{f+1} g_{f-1}^{-1}=e_{f+1}$.

Similarly we compute that

$$
e_{f+1} g_{f} e_{f-1} g_{f}^{-1} e_{f+1}=e_{f+1}=e_{f+1} e_{f} e_{f-1} e_{f} e_{f+1} .
$$

The case $\chi=g_{f-1}$ goes similarly.

For (f), one has to show that $\operatorname{tr}(a b)=\operatorname{tr}(b a)$ for any $a, b \in C_{f+1}$. We will use the fact that $C_{f+1}$ is the linear span of elements of the form $c \chi d$ with $c, d \in C_{f}$ and $\chi \in\left\{1, g_{f}, e_{f}\right\}$. Firstly, assume that $a \in C_{f}$ and $b \in C_{f+1}$. Then it follows from the induction assumption on tr, the bimodule property [see below (3.2)] and (3.3),

$$
\operatorname{tr}(a b)=\operatorname{tr}\left(a \varepsilon_{f}(b)\right)=\operatorname{tr}\left(\varepsilon_{f}(b) a\right)=\operatorname{tr}(b a) .
$$

By semisimplicity of $I_{f+1}$, there exists a central idempotent $z \in C_{f+1}$ such that ${ }_{z} C_{f+1}=I_{f+1}$. So if $a \in I_{f+1}$, one has $a b=(a z) b=a(z b)$, i.e. one can also assume $b$ to be in $I_{f+1}$. By linearity, it is enough to consider the case $a=c_{1} e_{f} c_{2}$ and $b=d_{1} e_{f} d_{2}$ with $c_{1}, c_{2}, d_{1}, d_{2} \in C_{f}$ (see Proposition 3.2). But then

$$
\operatorname{tr}(a b)=\operatorname{tr}\left(x c_{1} \varepsilon_{f-1}\left(c_{2} d_{1}\right) e_{f} d_{2}\right)=\operatorname{tr}\left(e_{f}\right) \operatorname{tr}\left(x \varepsilon_{f-1}\left(c_{2} d_{1}\right) \varepsilon_{f-1}\left(d_{2} c_{1}\right)\right) .
$$

Similarly, one shows

$$
\operatorname{tr}(b a)=\operatorname{tr}\left(e_{f}\right) \operatorname{tr}\left(x \varepsilon_{f-1}\left(d_{2} c_{1}\right) \varepsilon_{f-1}\left(c_{2} d_{1}\right)\right) .
$$

So the claim follows from induction assumption.

Now the only remaining case is if both $a, b \in C_{f+1} / I_{f+1}$. Observe that also elements of the form $d_{1} \chi d_{2}$ with $\chi \in\left\{1, e_{f}, g_{f}^{-1}\right\}$ and $d_{1}, d_{2} \in C_{f}$ span $C_{f+1}$. Hence we can assume without restriction of generality $a=c_{1} g_{f} c_{2}$ and $b=d_{1} g_{f}^{-1} d_{2}$ with $c_{1}, c_{2}$, $d_{1}, d_{2} \in C_{f}$. But then it follows from (e),

$$
\operatorname{tr}(a b)=\operatorname{tr}\left(g_{f} c_{2} d_{1} g_{f}^{-1} d_{2} c_{1}\right)=\operatorname{tr}\left(\varepsilon_{f}\left(g_{f} c_{2} d_{1} g_{f}^{-1}\right) d_{2} c_{1}\right)=\operatorname{tr}\left(\varepsilon_{f-1}\left(c_{2} d_{1}\right) \varepsilon_{f-1}\left(d_{2} c_{1}\right)\right) .
$$

By the same method, one shows

$$
\operatorname{tr}(b a)=\operatorname{tr}\left(\varepsilon_{f-1}\left(d_{2} c_{1}\right) \varepsilon_{f-1}\left(c_{2} d_{1}\right)\right) .
$$

The claim follows now from the fact that $\mathrm{tr}$ is a trace on $C_{f-1}$.

The following result shows how the algebraic structure of $C_{f}$ depends on $\operatorname{tr}$ (see also [BW, Theorem 3.7] and [W-2]).

Theorem 3.5. Let $C_{f}$ be the algebra over the field $\mathbf{C}(r, q)$ given by the relations above. Then we have for all $f \in \mathbf{N}$,

(a) $C_{f}$ is semisimple and it has the same decomposition into full matrix rings as the Brauer algebra $D_{f}$. In particular, $\hat{C}_{f}(x, 1) \cong D_{f}$ and we can write, as for the Brauer algebras

$$
C_{f} \cong \underset{\lambda \in \Gamma_{f}}{\oplus} C_{f, \lambda}
$$

where $C_{f, \lambda}$ is a full matrix ring and $\Gamma_{f}$ is the union of the set of all Young diagrams with $f, f-2, f-4, \ldots 1$ or 0 boxes.

(b) If $V_{\lambda}$ is a simple $C_{f, \lambda}$ module, it decomposes as a $C_{f-1}$ module in the form

$$
V_{\lambda}=\underset{\mu \leftrightarrow \lambda}{\oplus} V_{\mu}
$$


where $V_{\mu}$ is a simple $C_{f-1, \mu}$ module and $\mu$ runs through all diagrams which can be obtained by adding/removing a box to/from $\lambda$.

(c) The functional tr is a faithful trace on $C_{f}$. More precisely, there exists for each Young diagram $\lambda \in \Gamma_{f}$ a nonzero rational function $Q_{\lambda}$ independent of $f$ such that for a minimal idempotent $p_{\lambda} \in C_{f, \lambda}$ we have $\operatorname{tr}\left(p_{\lambda}\right)=Q_{\lambda}(r, q) / x^{f}$.

Proof. We proceed by induction on $f$. It is easy to check the claim for $C_{0}$ and $C_{1}$ with $Q_{[0]}=1$ and $Q_{[1]}=x$.

To show the claim for $C_{f+1}$ observe that $e_{f} C_{f-1} \cong C_{f-1}$ by induction assumption and Lemma 3.3. Hence one can define $\varepsilon_{f-1}$ and $\operatorname{tr}$ as in (3.2) and (3.3). With these definitions Theorem 1.1 can be applied for $A=C_{f-1}, B=C_{f}$ and $e=(1 / x) e_{f}$. By Proposition $3.2(\mathrm{~b}), I_{f+1} \cong$ End $_{C_{f-1}} C_{f}$ which is semisimple. Moreover, also $C_{f+1} / I_{f+1} \cong H_{f+1}\left(q^{2}\right)$ is semisimple, hence so is $C_{f+1}$. In particular, $C_{f+1} \cong I_{f+1} \oplus H_{f+1}\left(q^{2}\right)$.

By Theorem 1.1 (b), the simple components of $I_{f+1}$ are in 1-1 correspondence with the ones of $C_{f-1}$, hence they can be labelled by the elements of $\Gamma_{f-1}$. The simple components of the quotient are labelled by the elements of $\Lambda_{f+1}$. Hence the simple components of $C_{f+1}$ are labelled by $\Gamma_{f-1} \cup \Lambda_{f+1}=\Gamma_{f+1}$. The decomposition of a simple $C_{f+1}$ module into simple $C_{f}$ modules follows for $I_{f+1}$ modules from Theorem 1.1 (b) and induction assumption and for modules of the quotient from (2.2). So $C_{f}$ and $D_{f}$ have the same decomposition into full matrix rings and, in particular, the same dimensions over their corresponding ground fields.

It has already been shown that $I_{f+1}$ is semisimple, hence tr, as defined in (3.3) is a trace on $C_{f+1}$ by Lemma 3.4. If $p_{\lambda}$ is a minimal idempotent in $C_{f-1, \lambda}$, then $(1 / x) e_{f} p_{\lambda}$ is a minimal idempotent in $I_{f+1, \lambda}$ by Theorem 1.1 (b). By induction assumption we have

$$
\operatorname{tr}\left((1 / x) e_{f} p_{\lambda}\right)=(1 / x)^{2} \operatorname{tr}\left(p_{\lambda}\right)=(1 / x)^{2} Q_{\lambda}(r, q) / x^{f-1} .
$$

If $p_{\lambda}$ is a minimal idempotent of $C_{f+1, \lambda}$ which is not in $I_{f+1}$, one defines $Q_{\lambda}=\operatorname{tr}\left(p_{\lambda}\right) x^{f+1}$.

To show the faithfulness of tr note that the structure trace is faithful on Brauer's algebra $D_{f+1}(x) \cong \hat{C}_{f+1}(x, 1)$ for all but finitely many values of $x$. Hence if $p_{\lambda}$ is a minimal idempotent in $\left(\hat{C}_{f}(x, r)\right)_{\lambda}, \operatorname{tr}\left(p_{\lambda}\right)(x, 1) \neq 0$. In particular, $\hat{Q}_{\lambda}=x^{f} \operatorname{tr}\left(p_{\lambda}\right)$ is a nonzero rational function in $r$ and $x$. So after transforming back to the $q, r$ coordinates by (3.1) one obtains the desired nonzero rational function $Q_{\lambda}$ in $r$ and $q$.

Let us briefly sketch how one can deduce from the previous theorem the Kauffman link invariant along the lines of Jones' original derivation of his polynomial: Using the homomorphism of the braid groups into the algebras $C_{f}(r, q)$, determined by the map $\sigma_{i} \mapsto g_{i}$, tr induces a class function on the braid group $B_{\infty}$. It follows from Lemma $3.4(\mathrm{~b})$ and (d) that tr is a Markov trace. In order to get a link invariant, one needs to rescale tr such that $\operatorname{tr}\left(\sigma_{i}\right)=\operatorname{tr}\left(\sigma_{i}^{-1}\right)$. This is done easily by defining for a braid $\beta=\sigma_{m_{1}}^{n_{1}} \sigma_{m_{2}}^{n_{2}} \ldots \sigma_{m_{k}}^{n_{k}}$ the exponent sum $e(\beta)=\sum_{k} n_{k}$ and

$$
\operatorname{tr}(\beta)=r^{-e(\beta)} \operatorname{tr}(\beta) \text {. }
$$

As a consequence of Markov's theorem and Jones' work (see [Jo-2]) one obtains a link invariant $L_{\mathrm{tr}}$, defined by

$$
L_{\mathrm{tr}}(\hat{\beta}, f)=\hat{\operatorname{tr}}\left(\sigma_{1}\right)^{1-f} \hat{\operatorname{tr}}(\beta) .
$$


To show that this link invariant is the Kauffman polynomial (up to the change of parameters which we made) it suffices to check that the algebraically defined functional $\operatorname{tr}$ and the functional defined at the beginning of this section using Kauffman's $\widetilde{K}$ are the same. This follows from Lemmas 3.1 and 3.4.

\section{Semisimple and Unitary Representations of $C_{f}(r, q)$}

The crucial fact for proving semisimplicity of $C_{f}$ was the faithfulness of $\operatorname{tr}$. This can no longer be expected for the corresponding structure traces of the $\mathbf{C}$ algebras $C_{f}(r, q)$ in general. Even if tr is not faithful, one can still do a similar analysis in special cases for the quotient over the annihilator ideal.

So let $J_{f}(r, q)$ be the annihilator of $\operatorname{tr}$ in $C_{f}(r, q)$, i.e.

$$
J_{f}(r, q)=\left\{a \in C_{f}(r, q), \operatorname{tr}(a b)=0 \text { for all } b \in C_{f}(r, q)\right\} .
$$

As $C_{f+1}$ is the linear span of elements of the form $a \chi b$ with $\chi \in\left\{1, e_{f}, g_{f}\right\}$ and $a, b, c \in C_{f}$, it follows from $\operatorname{tr}(c(a \chi b))=\operatorname{tr}(c a b) \operatorname{tr}(\chi)$ (by Lemma 3.4) that

$$
J_{f}(r, q) \subset J_{f+1}(r, q) \text {. }
$$

So if $\pi_{\mathrm{tr}}^{(f)}$ denotes the representation of $C_{f}(r, q)$ coming from the GNS construction with respect to $\mathrm{tr}$, one has

$$
\pi_{\mathrm{tr}}^{(f)}\left(C_{f-1}(r, q)\right) \cong \pi_{\mathrm{tr}}^{(f-1)}\left(C_{f-1}(r, q)\right)
$$

Hence the algebra $\pi_{\mathrm{tr}}\left(C_{f}(r, q)\right)$ is well-defined regardless of the underlying representation space (which could be any $C_{f^{\prime}}(r, q), f^{\prime} \geqq f$ ). We will use a similar approach as for the Brauer algebras (see [W-2]) to determine the structure of $\pi_{\mathrm{tr}}\left(C_{f}(r, q)\right)$. The only additional difficulty comes from the fact that $H_{f}\left(q^{2}\right)$ is not semisimple if $q$ is a root of unity. As a first step we show the following

Lemma 4.1. (a) Let $r, q \in \mathrm{C}$. If $\pi_{\mathrm{tr}}\left(C_{f-1}(r, q)\right)$ and $\pi_{\mathrm{tr}}\left(C_{f}(r, q)\right)$ are both semisimple, then

$$
\pi_{\mathrm{tr}}\left(C_{f+1}(r, q)\right) \cong \pi_{\mathrm{tr}}\left(I_{f+1}(r, q)\right) \oplus \varrho_{f+1}\left(H_{f+1}\left(q^{2}\right)\right),
$$

where the first summand is isomorphic to the Jones basic construction for $\pi_{\mathrm{tr}}\left(C_{f-1}(r, q)\right) \subset \pi_{\mathrm{tr}}\left(C_{f}(r, q)\right)$ (which is semisimple) and the second summand is the image of a representation of $C_{f+1}(r, q)$ which factors over $I_{f+1}(r, q)$ (hence it can be regarded as a representation of $\left.H_{f+1}\left(q^{2}\right)\right)$. The second summand may of may not be semisimple.

(b) If $H_{m}\left(q^{2}\right)$ is semisimple for $m=1,2, \ldots f$, then so is $\pi_{\mathrm{tr}}\left(C_{f}(r, q)\right)$.

(c) $\varrho_{f}\left(H_{f-1}\left(q^{2}\right)\right)$ is isomorphic to a quotient of $\varrho_{f-1}\left(H_{f-1}\left(q^{2}\right)\right)$.

Proof. We proceed as in the proof of Theorem 3.5 with the algebra $C_{f}$ replaced by $\pi_{\mathrm{tr}}\left(C_{f}(r, q)\right)$. By definition, the trace is always faithful on $\pi_{\mathrm{tr}}\left(C_{f}(r, q)\right)$ so that we can use the same induction argument [Theorem (1.1) with $\pi_{\mathrm{tr}}\left(e_{f}\right)$ as idempotent] as before. Hence $\pi_{\mathrm{tr}}\left(I_{f+1}(r, q)\right)$ is isomorphic to the basic construction for $\pi_{\mathrm{tr}}\left(C_{f+1}(r, q)\right) \subset \pi_{\mathrm{tr}}\left(C_{f-1}(r, q)\right)$ which is semisimple. The quotient $\pi_{\mathrm{tr}}\left(C_{f+1}(r, q)\right) / \pi_{\mathrm{tr}}\left(I_{f+1}(r, q)\right)$ is obviously a quotient of the Hecke algebra $H_{f+1}\left(q^{2}\right)$. It has to split because $\pi_{\mathrm{tr}}\left(I_{f+1}(r, q)\right)$ is semisimple.

(b) follows from (a) by induction.

(c) As $I_{f-1}(r, q) \subset I_{f}(r, q),\left(C_{f-1}+I_{f} / I_{f}\right)(r, q)$ is isomorphic to a quotient of $\left(C_{f-1}+I_{f-1} / I_{f-1}\right)(r, q)$. The same holds for their images under $\pi_{\mathrm{tr}}$. 
So by the last lemma $\pi_{\mathrm{tr}}\left(C_{f}(r, q)\right)$ is semisimple for all $f \in \mathbf{N}$ if and only if at each stage the representations $\varrho_{f}\left(H_{f}\left(q^{2}\right)\right)$ are semisimple. It also shows that in this case the structure of $\pi_{\mathrm{tr}}\left(C_{f}(r, q)\right)$ is completely determined if one knows which representations of the Hecke algebra appear at each stage. In case that all the $\varrho_{f}$ 's are semisimple, this information will be encoded in the graph $\Gamma(r, q)$ whose vertices at the $f^{\text {th }}$ level are labelled by the simple components of $\varrho_{f}\left(H_{f}\left(q^{2}\right)\right)$. As for a Bratteli diagram, the edges are given by the decomposition of an irreducible $\varrho_{f}\left(H_{f}\left(q^{2}\right)\right)$ module into irreducible $H_{f-1}\left(q^{2}\right)$ modules. We also note that this is of course the same definition which Ocneanu uses for describing higher relative commutants of subfactors (the so-called principal graph) and we will show later that indeed our graphs do describe subfactors.

We shall see later that the $\varrho_{f}$ 's will always be direct sums of the representations $\pi_{\lambda}$ and $\pi_{\lambda}^{(k, l)}$ defined in Sect. 2. So we usually label the vertices of $\Gamma(r, q)$ by Young diagrams. The next lemma gives conditions when an $l$ regular diagram can appear in $\Gamma(r, q)$. The main difficulty consists of producing minimal idempotents of $C_{f}$ which are still well-defined for the chosen values of $r$ and $q$. More recently, a canonical set of idempotents of $C_{f}$ has been defined in [RW] which could also be used for the proof of the following lemma.

Lemma 4.2. Let $\mu \in \Gamma(r, q)$ be an l regular diagram with $f$ boxes and let $\lambda$ be an $l$ regular diagram obtained by adding/removing a box to/from $\mu$. Then $\lambda \in \Gamma(r, q)$ if and only if $Q_{\lambda}(r, q) \neq 0$. Moreover, one also has

(a) All subdiagrams of $\mu$ are in $\Gamma(r, q)$.

(b) There exists a minimal idempotent $p_{\lambda} \in C_{f+1, \lambda}$ such that also $p_{\lambda}(r, q)$ is welldefined.

(c) $\operatorname{tr}\left(p_{\lambda}\right)=Q_{\lambda}(r, q) / x^{f+1}$ and, if $|\lambda|>|\mu|, C_{f+1, \lambda}(r, q)$ is well-defined and splits as a direct summand.

Proof. The proof goes by induction on $f$, the number of boxes of $\mu$. By definition, $\pi_{\mu}\left(C_{f}(r, q)\right)$ is a direct summand of $\pi_{\mathrm{tr}}\left(C_{f}(r, q)\right)$. But as

$$
\pi_{\mu \mid C_{f-1}(r, q)} \cong \bigoplus_{\eta<\mu} \pi_{\eta},
$$

all subdiagrams $\eta$ of $\mu$ with $f-1$ boxes are in $\Gamma(r, q)$ by Lemma 4.1 (c). The claim in (a) follows from this and induction assumption.

By induction assumption and (a), there exists a minimal idempotent $p_{\mu} \in C_{f, \mu}$ such that $p_{\mu}(r, q)$ is well-defined. By Theorem 3.6 there exists for each subdiagram $\eta$ of $\mu$ with $f-1$ boxes a minimal idempotent $p_{\eta} \in C_{f+1, \eta}$ such that

$$
\left(p_{\mu} I_{f+1} p_{\mu}\right)(r, q) \cong \underset{\eta<\mu}{\bigoplus} \mathbf{C}(r, q) p_{\eta} .
$$

On the other hand, using the symmetry of the Bratteli diagram for Jones' basic construction (Theorem 1.1) and Lemma 4.1 (a) we also have

$$
\pi_{\mathrm{tr}}\left(\left(p_{\mu} I_{f+1} p_{\mu}\right)(r, q)\right) \cong \underset{\eta<\mu}{\bigoplus} \mathbf{C} .
$$

Hence $\pi_{\mathrm{tr}}$ is faithful on $p_{\mu} I_{f+1} p_{\mu}(r, q)$ and $p_{\eta, f+1}(r, q)$ is well-defined for all $\eta<\mu$ by Proposition 1.3 (b) (with $B=p_{\mu} I_{f+1} p_{\mu}$ ). In particular, $p_{\mu} I_{f+1} p_{\mu}(r, q)$ is semisimple and the quotient $p_{\mu} C_{f+1} p_{\mu}(r, q) / p_{\mu} I_{f+1} p_{\mu}(r, q)$ has to split. As $\lambda$ is $l$ regular, the central idempotent $z_{\lambda}\left(q^{2}\right)$ does exist in $H_{f+1}\left(q^{2}\right)$ and one can take for $p_{\lambda}$ the image of $p_{\mu} z_{\lambda}$ in the quotient (which splits). (c) now follows from this and Lemma 1.4 (c). By 
Lemma 1.4 (b), $C_{f+1, \mu}$ appears as a direct summand in $\pi_{\mathrm{tr}}\left(C_{f+1}(r, q)\right)$ if and only if $\operatorname{tr}\left(p_{\lambda}\right)(r, q) \neq 0$, which shows the main statement.

In order to compute $\Gamma(r, q)$ the previous lemma suggests the definition of the graph $\widetilde{\Gamma}(r, q) \subset \Lambda$, defined inductively by $[0] \in \widetilde{\Gamma}(r, q)$ and $\lambda \in \widetilde{\Gamma}(r, q)$ if

(a) $Q_{\lambda}(r, q) \neq 0$ and

(b) there is at least one subdiagram of $\lambda$ with $|\lambda|-1$ boxes which is in $\tilde{\Gamma}(r, q)$.

Observe that by the previous lemma an $l$ regular diagram $\lambda$ is in $\Gamma(r, q)$ if and only if it is in $\tilde{\Gamma}(r, q)$. For the general case one needs the next lemma. The semisimplicity assumption could be weakened to the assumption that both polynomials on the right-hand side of condition (2) are well-defined for the given values of $r$ and $q$ (from which semisimplicity would follow by Lemma 1.4). We will later use another method for proving it.

Lemma 4.3. Let $q^{2}$ be a primitive $l^{\text {th }}$ root of unity and assume that $\pi_{\mathrm{tr}}\left(C_{f}(r, q)\right)$ is se'misimple for all $f \in \mathbf{N}$. Then the hook diagram $\lambda=\left[l-k+1,1^{k-1}\right]$ is in $\Gamma(r, q)$ if and only if

(1) $\mu=\left[l-k+1,1^{k-2}\right] \in \Lambda_{l-1}(r, q)$ and

(2) $\bar{Q}_{\lambda}(r, q)=\left(Q_{\lambda}+Q_{\left[l-k+2,1^{k-2}\right]}\right)(r, q) \neq 0$.

Proof. As $\pi_{\mathrm{tr}}\left(C_{l}(r, q)\right)$ is semisimple, it is, by Lemma 4.1 (a), enough to consider the semisimple quotient $\bar{C}_{l}(r, q) \cong \pi_{\mathrm{tr}}\left(I_{l}(r, q) \oplus \bar{H}_{l}\left(q^{2}\right)\right.$, where $\bar{H}_{l}\left(q^{2}\right)$ is the maximal semisimple quotient of $H_{l}\left(q^{2}\right)$ (see Prop. 2.2). So $\lambda \in \Gamma(r, q)$ if and only if $\operatorname{tr}\left(\bar{p}_{\lambda}(r, q)\right) \neq 0$ for a minimal idempotent $p_{\lambda}(r, q)$ in the simple component of $\bar{C}_{l, \lambda}$ which is isomorphic to $\pi_{\lambda}^{(k, l)}\left(H_{l}\left(q^{2}\right)\right)$ by Lemma $1.4(\mathrm{~b})$.

By the restriction rules (see Proposition 2.2) and Lemma $4.1 \lambda \in \Gamma(r, q)$ only if $\mu=\left[l-k+1,1^{k-2}\right] \in \Gamma(r, q)$. As $\mu$ is $l$ regular, there exists a minimal idempotent $p_{\mu} \in C_{l-1, \mu}$ such that $p_{\mu}(r, q)$ is well-defined. that

One derives directly from the Bratteli diagram for $C_{l-1} \subset C_{l}$ (see Theorem 3.6)

$$
p_{\mu}=\sum_{\eta} p_{\eta}
$$

where $\eta$ runs through all diagrams which can be obtained by adding/removing a box to/from $\mu$. If $\eta$ is $l$ regular, $p_{\eta}(r, q)$ is well-defined and $\operatorname{tr}\left(p_{\eta}(r, q)\right)=Q_{\eta}(r, q) / x^{l}$ by Lemma 4.2. Observe that the only 2 diagrams among the $\eta$ 's which are not $l$-regular, are the hook diagrams $\lambda$ and $\left[l-k-2,1^{k-2}\right]$. Hence it follows from the Bratteli diagram for $\pi_{\mathrm{tr}}\left(C_{l-1}(r, q)\right) \subset \bar{C}_{l}(r, q)$ that

$$
\operatorname{tr}\left(\bar{p}_{\lambda}(r, q)\right)=\left(Q_{\mu}-\sum_{\eta l \text { regular }} Q_{\eta}\right)(r, q) / x^{l}
$$

Comparing this with the decomposition of $p_{\mu}$ one obtains $\operatorname{tr}\left(\bar{p}_{\lambda}(r, q)\right)$ $=\left(Q_{\lambda}+Q_{\left[l-k-2,1^{k-2}\right]}\right)(r, q) / x^{l}$.

Theorem 4.4. Let $\pi_{\mathrm{tr}}$ be the GNS construction with respect to $\operatorname{tr}$ and let $q^{2}$ be a primitive $l^{\text {th }}$ root of unity. Moreover, assume that $\pi_{\mathrm{tr}}\left(C_{f}(r, q)\right)$ is semisimple for all $f \in \mathbf{N}$. Then

(a) The isomorphisms in Proposition 3.2 also extend to $\pi_{\mathrm{tr}}\left(C_{f}(r, q)\right.$ ) (e.g. $\left.\pi_{\mathrm{tr}}\left(C_{f}(-r,-q)\right) \cong \pi_{\mathrm{tr}}\left(C_{f}(r, q)\right)\right)$. 
(b) Assume that $\tilde{\Gamma}(r, q)$, as defined before Lemma 4.3, does not contain a hook diagram with $l-1$ boxes. Then $\widetilde{\Gamma}(r, q)=\Gamma(r, q)$ with edges inherited from the Young lattice and it only contains $l-1$ regular diagrams.

(c) If $\widetilde{\Gamma}(r, q)$ contains only one hook diagram $\mu$ with $l-1$ boxes with, say, $\mu=\left[l-k, 1^{k-1}\right]$ and it does not contain its successor $\left[l-k, 2,1^{k-2}\right], \Gamma(r, q)$ consists of all l regular diagrams in $\widetilde{\Gamma}(r, q)$ and, if $\bar{Q}_{\lambda}(r, q) \neq 0$ (with $\widetilde{Q}_{\lambda}$ as in the last lemma), also of the diagram $\lambda=\left[l-k+1,1^{k-1}\right]$. The edges of $\Gamma(r, q)$ are exactly those inhertited from the Young lattice.

(d) These graphs completely determine the decomposition of $\pi_{\mathrm{tr}}\left(C_{f}(r, q)\right)$ into a direct sum of full matrix rings. The weight vector of $\operatorname{tr}$ for $\pi_{\mathrm{tr}}\left(C_{f}(r, q)\right)$ is equal to $\left(Q_{\lambda}(r, q) / x^{f}\right)_{\lambda \in \Gamma(r, q)_{f}}$ except for the special diagram in (c) where one has to take $\bar{Q}_{\lambda}(r, q)$.

Proof. Let $\Phi$ be as in the proof of Proposition 3.2 (c). For (a), it is enough to show that $\operatorname{tr} \circ \Phi$ is the structure trace on the algebra on which $\Phi$ is defined. This follows by induction using Lemma 3.4 (b) and (d).

(b) The proof goes by induction on $f$ with $f=0$ and $f=1$ being trivial. By induction assumption, we know that the simple components of $\varrho_{f}\left(C_{f}(r, q)\right)$ are labelled by $l-1$ regular diagrams. But then $\varrho_{f+1}\left(H_{f+1}\left(q^{2}\right)\right)$ has to be semisimple and all its irreducible components have to be labelled by $l$ regular diagrams by Proposition 2.1 (b). By our assumption and Lemma 4.2 only $l-1$ regular diagrams can appear.

For (c), one uses essentially the same strategy where one only has to consider the cases $f=l$ and $f=l+1$ separately. By Lemma 4.3 and our assumptions, $\varrho_{l}\left(C_{l}(r, q)\right)$ is semisimple and its simple components are labelled either by $l-1$ regular diagrams or by diagrams containing $\left[l-k, 1^{k-1}\right]$. The diagram $\left[l-k, 2,1^{k-2}\right]$ is $l$ regular, hence it does not appear in $\Gamma(r, q)$ by our assumptions and Lemma 4.2. By Lemma 4.3 the diagram $\left[l-k+1,1^{k-1}\right]$ only appears if $\bar{Q}_{\left[l-k+1,1^{k-1}\right]}(r, q) \neq 0$.

By Proposition 2.2, $\varrho_{l+1}$ has to be semisimple with its simple components labelled by $l-1$ regular diagrams none of which contains $\left[l-k, 1^{k-1}\right]$. Now one can show as for (b) that only $l-1$ regular diagrams can appear in $\varrho_{f}\left(C_{f}(r, q)\right)$ for $f>l$.

Let us demonstrate at an example how one can determine the structure of $\pi_{\mathrm{tr}}\left(C_{f}(r, q)\right), f \in \mathbf{N}$ from $\Gamma(r, q)$. It will be shown in Sect. 6 that for $q=e^{2 \pi i / 7}$ and $r=q^{2}$, the graph $\Gamma(r, q)$ is given by

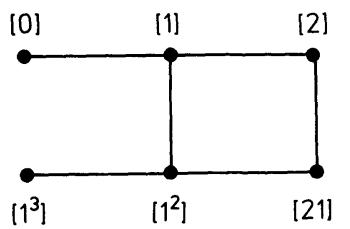

Fig. 6

It follows from the definition of $\Gamma(r, q)$ and the last theorem that the Bratteli diagram for the $\pi_{\mathrm{tr}}\left(C_{f}(r, q)\right)$ is of the following form (where diagrams with $f$ boxes appear in the $(f+1)^{\text {st }}$ line for the first time) 


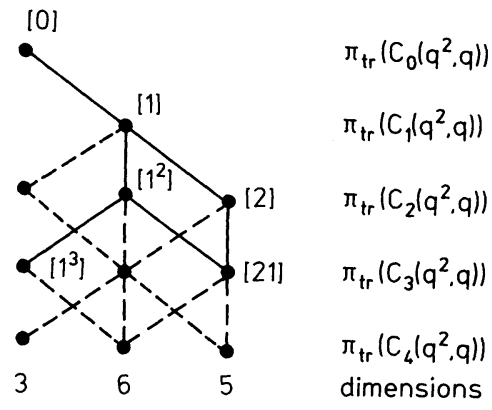

Fig. 7

In the rest of this section, we will give conditions when representations of $C_{f}(r, q)$ on a Hilbert space can induce unitary representations of the braid group $B_{f}$. Slightly more general, a representation of $C_{f}(r, q)$ (or $H_{f}(q)$ ) will be called a $\mathrm{C}^{*}$ representation, if the corresponding representation of the braid group, induced by the homomorphism $\sigma_{i} \mapsto g_{i}$ maps the standard generators $\sigma_{i}$ to normal operators. An irreducible $\mathrm{C}^{*}$ representation is called nontrivial in this context if its image has dimension $>1$.

Observe that if $\varrho$ is a $\mathrm{C}^{*}$ representation, then $\varrho\left((1 / x) e_{i}\right)$ is a spectral projection of the normal element $\varrho\left(g_{i}\right)$, hence selfadjoint. Hence, as $\pi_{\mathrm{tr}}\left(I_{f+1}(r, q)\right)$ is isomorphic to the basic construction for $\pi_{\mathrm{tr}}\left(C_{f-1}(r, q)\right) \subset \pi_{\mathrm{tr}}\left(C_{f}(r, q)\right)$, Lemma 1.2 yields the following necessary conditions for $\pi_{\mathrm{tr}}\left(I_{f+1}(r, q)\right)$ to be a $\mathrm{C}^{*}$ representation:

(a) The restriction of $\pi_{\mathrm{tr}}$ to $C_{f}(r, q)$ has to be a $\mathrm{C}^{*}$ representation.

(b) All irreducible components of the restriction in (a) must have positive weights with respect to tr.

The following lemma will give more precise conditions for possible $C^{*}$ representations of $\pi_{\mathrm{tr}}\left(I_{f}(r, q)\right)$.

Lemma 4.5. Let $q, r \in \mathbf{C}$. If $q^{2}$ is a primitive $l^{l^{\text {th }}}$ root of unity, we assume the conditions for $\Gamma(r, q)$ as in Theorem 4.4 .

(a) If $r=-q$ or $r=q^{-1}, x=Q_{[1]}(r, q)=0$. In this case $\operatorname{tr}$ can not be defined.

(b) There are nontrivial $\mathrm{C}^{*}$ representations of $I_{f}(r, q), f \geqq 5$ factoring over $\pi_{\mathrm{tr}}$ only if

(b1) both $r$ and $q$ are real or if

(b2) $|r|=|q|=1$ or if

(b3) $r=q$ or $r=-q^{-1}$ or if

(b4) $r=q^{-3}$ or $r=-q^{3}$ and $q \in i \mathbf{R}$.

Proof. (a) is clear. For (b), let us first assume that $Q_{[2,1]}(r, q) \neq 0$, i.e. $\pi_{[21]}\left(H_{3}\left(q^{2}\right)\right)$ appears as a direct summand in $\pi_{\mathrm{tr}}\left(C_{3}(r, q)\right)$. By the restriction rule for $C_{f}(r, q)$ (see Theorem 4.4), any irreducible representation of $\pi_{\mathrm{tr}}\left(I_{f}(r, q)\right), f \geqq 5$, restricted to $C_{3}(r, q)$ contains a subrepresentation isomorphic to $\pi_{[2,1]}\left(C_{3}(r, q)\right) \cong \pi_{[2,1]}\left(H_{3}\left(q^{2}\right)\right)$. But by [W-1, Proposition 2.9] this can only be a nontrivial C* representation if $q^{2}$ is positive or if $\left|q^{2}\right|=1$.

By condition (b) the weights of tr have to be positive for any subrepresentation of $I_{f}$. This implies in particular for $I_{3}(r, q)$ that $Q_{[0]}(r, q) / x^{2}=1 / x^{2}>0$. One deduces from this easily that

$$
\frac{r-r^{-1}}{q-q^{-1}} \in \mathbf{R} \text {. }
$$


This is possible for $q$ real only if $r$ is also real and for $|q|=1$ only if $|r|=1$. This shows the claim under the condition $Q_{[2,1]}(r, q) \neq 0$. To complete the proof, we need the fact that

$$
Q_{[2,1]}(r, q)=\frac{r-r^{-1}+q^{3}-q^{-3}}{q^{3}-q^{-3}} \frac{r q-r^{-1} q^{-1}}{q-q^{-1}} \frac{r q^{-1}-r^{-1} q}{q-q^{-1}} .
$$

This will be shown in the next section using solutions of the quantum YangBaxter equations. It can also be shown by elementary means by constructing minimal idempotents in $C_{3,[2,1]}(r, q)$ and by computing their trace. Observe that $Q_{[2,1]}(r, q)=0$ only if

$$
\begin{aligned}
& r=q \text { or } r=-q^{-1}, \text { which is case (b3), } \\
& r=q^{-1} \text { or } r=-q, \text { which is case (a), or if } \\
& r=q^{-3} \text { or } r=-q^{3} .
\end{aligned}
$$

If $r=q^{-3}$, we also have $Q_{[2]}(r, q)=0$ (see Theorem 5.5 or compute it directly). Hence $\pi_{\mathrm{tr}}\left(g_{1}\right)$ only has 2 different spectral projections with spectral values $r^{-1}$ and $q^{-1}$. It follows that $\pi_{\mathrm{tr}}\left(C_{f}(r, q)\right)$ is isomorphic to a quotient of the Hecke algebra $H_{f}\left(q^{4}\right)$. So again by [W-1] $q^{4}$ has to be either real and positive or its absolute value has to be equal to 1 . If $r=-q^{3}, Q_{\left[1^{2}\right]}(r, q)=0$ and one can show the statement as before.

Recall that the characteristic values of the $g_{i}$ 's are $q,-q^{-1}$ and $r^{-1}$. By the result above we have therefore for any $\mathrm{C}^{*}$ representation $\varrho$ of $C_{f}(r, q), f \geqq 5$ that either $\varrho\left(g_{i}\right)$ is unitary (for $|r|=|q|=1$ ) or selfadjoint (for $r$ and $q$ real).

So one can already define an involution for $C_{f}(r, q)$ which is compatible with the * operation for $\mathrm{C}^{*}$ representations. More precisely, if $|r|=|q|=1$, one defines the conjugate linear map ${ }^{\dagger}$ on $C_{f}(r, q)$ by

and

$$
g_{i}^{\dagger}=g_{i}^{-1} \quad i=1,2, \ldots f-1
$$

$$
(\alpha a b)^{\dagger}=\bar{\alpha} b^{\dagger} a^{\dagger} \quad \text { for } \quad a, b \in C_{f}(r, q), \quad \alpha \in \mathbf{C} .
$$

It is checked easily that ${ }^{\dagger}$ is compatible with the generating relations of $C_{f}(r, q)$, hence it is well defined.

Similarly, if both $r$ and $q$ are real, one defines ${ }^{\dagger}$ as above where the first line is replaced by

$$
g_{i}^{\dagger}=g_{i} \quad i=1,2, \ldots f-1 .
$$

Note that one has in both cases

$$
e_{i}^{\dagger}=e_{i} \quad i=1,2, \ldots f-1 .
$$

It follows from these definitions that a representation $\varrho$ of $C_{f}(r, q)$ on a Hilbert space is a $C^{*}$ representation if and only if $\varrho\left(g_{i}\right)^{*}=\varrho\left(g_{i}^{\dagger}\right)$ for $i=1,2, \ldots f-1$.

The operation ${ }^{\dagger}$ is also used to define a sesquilinear form on $C_{f}(r, q)$ by

$$
\langle a, b\rangle=\operatorname{tr}\left(b^{\dagger} a\right) .
$$

In the following we will always define the representation coming from the GNS construction with respect to this sesquilinear form.

Theorem 4.6. $\pi_{\mathrm{tr}}$ is a $\mathrm{C}^{*}$ representation of $C_{\infty}(r, q)$ if and only if

(a) $\varrho_{f}$ is a $\mathrm{C}^{*}$ representation of $H_{f}\left(q^{2}\right)$ for all $f \in \mathbf{N}$,

(b) $Q_{\lambda}(r, q) / x^{|\lambda|}>0$ for all $\lambda \in \Gamma(r, q)$. 
Similarly, $\pi_{\mathrm{tr}}$ is a $\mathrm{C}^{*}$ representation of $\mathrm{C}_{f_{0}}(r, q)$ if and only if the conditions (a) and (b) hold for $1 \leqq f \leqq f_{0}$ and for all diagrams in $\Gamma(r, q)$ with $f_{0}$ boxes at the most.

Proof. If $\pi_{\mathrm{tr}}$ is a $\mathrm{C}^{*}$ representation, condition (a) follows immediately and condition (b) follows from Lemma 1.2 and Theorem 4.4 .

The converse is shown by induction on $f$. We first show that if $\pi_{\mathrm{tr}}$ is a $\mathrm{C}^{*}$ representation of $C_{f-1}(r, q)$ and $C_{f}(r, q)$, conditions (a) and (b) imply that the sesquilinear form $\langle$,$\rangle , defined in (4.4), is positive semidefinite on I_{f+1}(r, q)$. By Lemma 1.2, the basic construction for $2 \mathrm{C}^{*}$ algebras $A \subset B$ is a $\mathrm{C}^{*}$ algebra if $\mathrm{tr}$ is faithful and positive. Hence $\pi_{\mathrm{tr}}\left(I_{f+1}(r, q)\right)$ is a $\mathrm{C}^{*}$ algebra by (a) and (b). As the weight vector of $\operatorname{tr}$ on $\pi_{\mathrm{tr}}\left(I_{f+1}(r, q)\right)$ is equal to $\left(Q_{\mu}(r, q) / x^{f+1}\right)_{\mu \in \Gamma_{f-1}(r, q)}$, which is positive by assumption (because $f-|\mu|$ is even), $\langle$,$\rangle is positive semidefinite on$ $I_{f+1}(r, q)$ by $(1.5)$.

By (a), also $\varrho_{f+1}$ is a $C^{*}$ representation of $H_{f+1}\left(q^{2}\right)$. As the weights $Q_{\lambda}(r, q) / x^{|\lambda|}$ corresponding to the simple components of $\varrho_{f+1}$ are positive, tr also induces a positive definite sesquilinear form on $\varrho_{f+1}\left(H_{f+1}\left(q^{2}\right)\right)$. As $\operatorname{tr}(a b)=\operatorname{tr}(0)=0$ for $a \in \pi_{\mathrm{tr}}\left(I_{f+1}(r, q)\right)$ and $b \in \varrho_{f+1}\left(H_{f+1}\left(q^{2}\right)\right)$, tr induces a positive definite sesquilinear form on $\pi_{\mathrm{tr}}\left(C_{f+1}(r, q)\right)$. Hence $\pi_{\mathrm{tr}}$ represents $C_{f+1}(r, q)$ on a Hilbert space.

Assume that $|r|=|q|=1$ and let $a, b \in C_{f+1}(r, q)$.

$$
\left\langle a, g_{i}^{\dagger} b\right\rangle=\operatorname{tr}\left(b^{\dagger} g_{i} a\right\rangle=\left\langle g_{i} a, b\right\rangle .
$$

The same identity factors through $\pi_{\mathrm{tr}}$. Hence, as $\langle$,$\rangle is an inner product on$ $\pi_{\mathrm{tr}}\left(C_{f+1}(r, q)\right), \pi_{\mathrm{tr}}\left(g_{i}^{\dagger}\right)$ is the adjoint of $\pi_{\mathrm{tr}}\left(g_{i}\right)$. By remarks after (4.3), this means that $\pi_{\mathrm{tr}}$ is a $\mathrm{C}^{*}$ representation.

Corollary 4.7. Assume $|r|=|q|=1$ and $r \neq \pm q^{ \pm 1}$. Moreover, assume that $q^{2}=e^{t 2 \pi i}$, $0<t<1 / 2$ and that there exists $m \in \mathbf{N}$ such that

$$
1 / m<|t|<1 /(m-1) \text {. }
$$

Then $\pi_{\mathrm{tr}}$ is a $\mathrm{C}^{*}$ representation of $C_{\infty}(r, q)$ only if all diagrams in $\Gamma(r, q)$ are $m$ regular.

Proof. It is checked easily that $q^{2}$ can not be a primitive $k^{\text {th }}$ root of unity for $k=2,3, \ldots m, m+1$. Now if the condition above did not hold, there would exist an $(m+1)$ regular diagram $\lambda \in \Gamma(r, q)$ with, say, $f$ boxes which is not $m$ regular. By Theorem $4.4 \pi_{\lambda}\left(H_{f}\left(q^{2}\right)\right)$ is a direct summand in $\pi_{\mathrm{tr}}\left(C_{f}(r, q)\right)$. But by [W-1, Proposition 2.9] this summand can never belong to a $C^{*}$ representation.

\section{Quantum Groups and Markov Traces}

In the last section we have reduced questions about semisimplicity and positivity structures of $C_{f}(r, q)$ to faithfulness and positivity of the structure trace tr. To settle these questions, we need representations of $C_{f}(r, q)$ which use solutions of the quantum Yang-Baxter equation (QYBE).

Let $V$ be a finite dimensional vector space. Let $R(x)$ be a matrix in End $V \otimes V$, depending on a parameter $x$ and let $R_{1}=R \otimes 1$ and $R_{2}=1 \otimes R$ be matrices in End $V \otimes V \otimes V$. Then $R(x)$ is a solution of the QYBE if

$$
R_{1}(x) R_{2}(x y) R_{1}(y)=R_{2}(y) R_{1}(x y) R_{2}(x) .
$$

This is the version of the QYBE for the matrices $\check{R}$ in [Ji1]. It is easy and well-known how to deduce this version from the original one (for the computation see for 
instance [W-3]). The most successful method for obtaining solutions of the QYBE was the quantum group approach (see Drinfeld [D] and Jimbo [Ji1-3].

A quantum group is a $q$ deformation of the universal enveloping algebra of a finite or affine Lie algebra in the sense that its Chevalley generators satisfy relations depending on a parameter $q$ which reduce to the usual relations for $q=1$. We write down these relations for sake of completeness (see also [Ji-3, Ro or L]).

Let $A=\left(a_{i j}\right)_{1 \leqq i, j \leqq l}$ be a generalized Cartan matrix belonging to a finite or affine Lie algebra $\mathfrak{g}$. Then $A$ is symmetrizable, i.e. there exist nonzero integers $d_{i}$, $i=1,2, \ldots l$ such that $d_{i} a_{i j}=d_{j} a_{j i}$. Then, for $q \in \mathbf{C}$, one defines the quantum group $U_{q} \mathfrak{g}$ by generators $X_{i}^{+}, X_{i}^{-}, k_{i}, k_{i}^{-1}(1 \leqq i \leqq l)$ and relations

$$
\begin{aligned}
& k_{i} k_{j}=k_{j} k_{i}, \quad k_{i} k_{i}^{-1}=k_{i}^{-1} k_{i}=1, \\
& k_{i} X_{j}^{ \pm} k_{i}^{-1}=q^{ \pm d_{i} a_{i j} / 2} X_{j}^{ \pm}, \\
& {\left[X_{i}^{+}, X_{j}^{-}\right]=\delta_{i j} \frac{k_{i}^{2}-k_{i}^{-2}}{q^{d_{i}}-q^{-d_{i}}},} \\
& \sum_{v=0}^{1-a_{i j}}(-1)^{v} \frac{\left(1-a_{i j}\right)_{q_{i}^{d_{i}} !}}{[v]_{q^{d_{i}} !\left[1-a_{i j}-v\right]_{q^{d_{i}} !}}}\left(X_{i}^{ \pm}\right)^{1-a_{i j}-v}\left(X_{j}^{ \pm}\right)\left(X_{i}^{ \pm}\right)^{v}=0 \quad i \neq j,
\end{aligned}
$$

where

$$
[m]_{t} !=\prod_{j=1}^{m} \frac{t^{j}-t^{-j}}{t-t^{-1}} \quad \text { for any } \quad t \in \mathbf{C} .
$$

Observe that by setting $e_{i}=X_{i}^{+}, f_{i}=X_{i}^{-}$and by setting formally $k_{i}=q^{d_{i} h_{i} / 2}$, one obtains the relations among the Chevalley generators for the classical universal enveloping algebra in the limit $q \rightarrow 1$.

$U_{q} \mathfrak{g}$ also has a comultiplication $\Delta$ defined by

$$
\Delta\left(X_{i}^{ \pm}\right)=k_{i} \otimes X_{i}^{ \pm}+X_{i}^{ \pm} \otimes k_{i}^{-1}, \quad \text { and } \quad \Delta\left(k_{i}\right)=k_{i} \otimes k_{i} .
$$

The general representation theory of such quantum groups has been studied in [Ro, Lu]. If $q$ is not a root of unity, it is similar to the one of the corresponding classical algebra. It has been shown that one obtains for each finite dimensional representation of a quantum group on a finite dimensional vector space $V$ a solution $R(x) \in$ End $V \otimes V$ of the QYBE depending on a parameter $x$ (this follows from Drinfeld's universal $R$ matrix ; see [D]). It can be understood as a deformation of the flip $P$ (i.e. $P(v \otimes w)=w \otimes v)$, which lies in the commutant of the second tensor power of the given representation of the quantum group.

In the following, we will need the $R$ matrix for the standard representation of quantized $s o_{n+1}$ with $n+1$ odd (which is obtained as a special case from the $R$ matrix of the corresponding untwisted affine Lie algebra). The same procedure also goes through for the other classical Lie algebras (see [Re]). Let $V$ be an $n+1$ dimensional vector space with $n+1$ odd. It will be convenient to label a basis $\left\{v_{i}, i \in I\right\}$ of $V$ by the set

$$
I=\{-n+1,-n+3, \ldots-1,0,1,3, \ldots n-1\} .
$$

Observe that this is the set of eigenvalues of a matrix corresponding to the sum of positive roots of $s o_{n+1}$ with respect to a suitably normalized invariant bilinear form. For other Lie algebras one chooses the index set in the same way (where the value 0 appears twice for $s o_{n+1}$ with $n+1$ even). Let $\breve{R}(x)$ be Jimbo's solution of the QYBE for type $B_{n / 2}^{(1)}$ (see [Ji1]). Following Turaev [T] one extracts from this the matrix 
$R=(k \xi)^{-1} \check{R}(0)$ which has the following form (with $\left(f_{i j}\right)_{i, j \in I}$ a set of matrix units for End $V$ ):

$$
\begin{aligned}
R= & \sum_{i \neq 0}\left(q f_{i, i} \otimes f_{i, i}+q^{-1} f_{i,-i} \otimes f_{-i, i}\right)+f_{0,0} \otimes f_{0,0}+\sum_{i \neq j,-j} f_{i, j} \otimes f_{j, i} \\
& +\left(q-q^{-1}\right)\left(\sum_{i<j} f_{i, i} \otimes f_{j, j}-\sum_{j<-i} q^{(i+j) / 2} f_{i, j} \otimes f_{-i,-j}\right) .
\end{aligned}
$$

It can be checked either by an explicit computation or by formulas in [Ji-1] that

$$
\begin{aligned}
R^{-1}= & \sum\left(q^{-1} f_{i, i} \otimes f_{i, i}+q f_{i,-i} \otimes f_{-i, i}\right)+f_{0,0} \otimes f_{0,0}+\sum_{i \neq j,-j} f_{i j} \otimes f_{j i} \\
& +\left(q-q^{-1}\right)\left(\sum_{j>-i} q^{(i+j) / 2} f_{i, j} \otimes f_{-i,-j}-\sum_{i>j} f_{i, i} \otimes f_{j, j}\right) .
\end{aligned}
$$

Let us also define the quantity $x$ (which corresponds to the parameter $x$ in the definition of $C_{f}$ for the special choice $r=q^{n}$ ) by

$$
x=\frac{q^{n}-q^{-n}+q-q^{-1}}{q-q^{-1}}=\sum_{i \in I} q^{i} .
$$

Let $F$ be the matrix defined on $V \otimes V$ by

Then

$$
F=\sum_{i, j} q^{(i+j) / 2} f_{i, j} \otimes f_{-i,-j}
$$

$$
F^{2}=x F
$$

and $1 / x F$ is a projection onto the subspace of $V \otimes V$ spanned by the vector $\sum_{i \in I} q^{i / 2}\left(v_{i} \otimes v_{-i}\right)$. It is easy to check that

$$
R-R^{-1}=\left(q-q^{-1}\right)(1-F) .
$$

One can now define elements $R_{i} \in \otimes^{\infty} M_{n+1}$ by

$$
R_{i}=1 \otimes \ldots 1 \otimes R \otimes 1 \otimes \ldots,
$$

where $R$ is in the $i^{\text {th }}$ and $(i+1)^{\text {st }}$ position in $\otimes{ }^{\infty} M_{n+1}$.

The following result has already been mentioned in $[\mathrm{T}]$ and $[\mathrm{Re}]$.

Lemma 5.1. If $q \neq 0, \pm 1$, the algebra generated by the $R_{i}$ 's, $i=1,2, \ldots f-1$, is a quotient of $C_{f}\left(q^{n}, q\right)$.

Proof. One only needs to show that the map $\Phi: g_{i} \mapsto R_{i}$ extends to a homomorphism. $\left(\mathrm{B}_{2}\right)$ is easy to check while (B1) follows from (5.1) with $x=y=0$. It seems almost impossible to check (B1) directly by elementary methods. It follows from (5.4) and (D) that $\Phi\left(e_{i}\right)=F_{i}$. Hence to check (R2) it is enough to show

$$
(1 \otimes F)(R \otimes 1)(1 \otimes F)=q^{n}(1 \otimes F),
$$

which is a modestly tedious, but straightforward computation. Finally, (R2) follows from $R F=q^{-n} F$, which follows easily from the fact that $F$ is a rank 1 idempotent.

Let $A(t)$ be a finite dimensional complex algebra depending on a parameter $t$ which acts on a finite dimensional vector space $W$ such that 
(a) $A(t) \cong A(1)$ is semisimple for all but finitely many values of $t$,

(b) if $A(t)$ is semisimple, the entries of the matrices representing the central idempotents depend continously on $t$ and

(c) there exists a matrix $D$ acting on $W$ which commutes with $A(t)$ for all values of $t$.

Lemma 5.2. Let $\operatorname{Tr}$ be the usual trace on matrices. With the notations above, the functional $\psi$, defined by $\psi(a)=\operatorname{Tr}(D a)$, is a trace on $A(t)$ for all $t$ such that

(a) the weight vector of $\psi$ does not depend on $t$ as long as $A(t)$ is semisimple and

(b) $\pi_{\psi}(A(t))$ is semisimple for all values of $t$, where $\pi_{\psi}$ is the representation defined by the GNS construction.

Proof. As any 2 elements $a, b \in A(t)$ commute with $D$, we have

$$
\psi(a b)=\operatorname{Tr}(D a b)=\operatorname{Tr}(a D b)=\operatorname{Tr}(b a D)=\psi(b a) .
$$

Obviously, $D$ as a diagonal matrix has a spectral decomposition $D=\sum_{\alpha} \alpha E_{\alpha}$ with $\alpha$ ranging over the eigenvalues of $D$. As $D$ commutes with $A(t),{ }^{\alpha} E_{\alpha} z(t)$ is an idempotent. As $t \mapsto \operatorname{Tr}\left(z(t) E_{\alpha}\right) \in \mathbf{N}$ is continuous, it has to be constant. Hence

$$
\psi(z(t))=\operatorname{Tr}(D(z(t)))=\sum_{\alpha} \alpha \operatorname{Tr}\left(E_{\alpha} z(t)\right)
$$

does not depend on $t$. Hence also the entries of the weight vector of $\psi$, which are given by $\psi(z(t))$ divided by the corresponding dimension do not depend on $t$ by assumption (a).

To prove statement (b), let $a \in \operatorname{rad}(A(t))$ and let $b \in A(t)$ be arbitrary. As $D$ commutes with $A(t), D a b$ is also nilpotent. Hence $\psi(a b)=\operatorname{Tr}(D a b)=0$ for any $b \in A(t)$. This shows that $a$ is in the kernel of $\pi_{\psi}$ by definition of GNS construction.

The previous lemma will now be applied to the following situation: Let $A(t)=\Phi\left(C_{f}\left(t^{n}, t\right)\right)$, with $\Phi$ as in the proof of Lemma 3.1, acting on $W=V^{\otimes f}$. In order to check that $A(1)$ is isomorphic to Brauer's $D_{f}(n+1)$ if $f<n$, we introduce the basis $\left\{\vec{w}_{j}, j \in I\right\}$ for $V$ given by

Observe that

$$
\begin{aligned}
& \vec{w}_{j}=\frac{1}{\sqrt{2}}\left(\vec{v}_{j}+i \vec{v}_{-j}\right) \text { for } j>0, \\
& \vec{w}_{j}=\vec{v}_{0} \quad \text { for } j=0 \text {, } \\
& \vec{w}_{-j}=\frac{1}{\sqrt{2}}\left(\vec{v}_{-j}-i \vec{v}_{j}\right) \text { for } j>0 \text {. }
\end{aligned}
$$

$$
i\left(\vec{w}_{j} \otimes \vec{w}_{j}+\vec{w}_{-j} \otimes \vec{w}_{-j}\right)=\vec{v}_{j} \otimes \vec{v}_{-j}+\vec{v}_{-j} \otimes \vec{v}_{j} .
$$

Hence $F$, which for $q=1$ is a multiple of the orthogonal projection onto the vector $\sum_{i \in I} v_{i} \otimes v_{-i}$ transforms to $n+1$ times the projection onto the vector $\sum_{j \in I} \vec{w}_{j} \otimes \vec{w}_{j}$. Obviously, the $R_{i}$ 's, which for $q=1$ only permute the factors of $V^{\otimes f}$, do not change under this basis transformation. It follows now by well-known classical results (see [Br or Wy]) that the $R_{i}$ 's and $F \otimes 1^{\otimes f-2}$ generate the centralizer $\varrho^{\otimes f}(O(n+1))^{\prime}$ of the $f^{\text {th }}$ tensor product representation of the orthogonal group $O(n+1)$. Moreover, if $f<n$, the centralizer algebra is isomorphic to the algebra $D_{f}(n+1)$ (see for instance [Wy, p. 149]). The semisimplicity of the centralizer algebras can be seen directly from the fact that the generating matrices are selfadjoint (or see also [W-2]). 
As $C_{f}\left(t^{n}, t\right)$ is an algebra over $\mathbf{C}(t)$, its central idempotents can be written as a sum of products of the generators $g_{i}$ and $e_{i}$ with coefficients in $\mathbf{C}(t)$ (which, in particular, are continuous functions in $t$ except at poles). Hence, as the matrices for the $R_{i}$ 's and $F_{i}$ 's depend continuously on $t$, so do the matrices for the central idempotents for $A(t)$. Hence $A(t)$ satisfies assumptions (a) and (b) before Lemma 5.2.

Let $D$ be an $(n+1) \times(n+1)$ diagonal matrix with diagonal entries $\alpha_{1}, \alpha_{2}, \ldots \alpha_{n+1}$. The matrix $D^{\otimes f}$ acts on $W=V^{\otimes f}$ in the usual way by $D^{\otimes f}\left(v_{1} \otimes v_{2} \otimes \ldots v_{f}\right)$ $=D v_{1} \otimes D v_{2} \otimes \ldots D v_{f}$. To obtain a trace $\psi$ on $A(t)$ as in Lemma $5.2, D^{\otimes f}$ has to commute with $A(t)$ for all values of $t$, or equivalently $A(t)$ has to leave invariant all eigenspaces of $D^{\otimes f}$ which are defined by

$$
V_{\alpha}^{\otimes f}=\operatorname{span}\left\{\vec{v}_{i_{1}} \otimes \ldots \vec{v}_{i_{k}} \in V^{\otimes f}, \prod_{j=1}^{f} \alpha_{i_{j}}=\alpha\right\} .
$$

It is easy to check that $R$ leaves invariant the eigenspaces of $D^{\otimes 2}$ if $\alpha_{-i}=\alpha_{i}^{-1}$ for any choice of $q$ (or rather $t$ ). From this it follows easily that $D^{\otimes f}$ commutes with $R_{i}$, $i=1,2, \ldots f-1$ and hence with $A(t)$.

So if the eigenvalues of $D$ have the property stated above, we obtain a trace on $A(t)$ from the functional $\psi$ on $M_{n+1}^{\otimes f}$ (essentially a product state) defined by

$$
\psi(a)=\operatorname{Tr}\left(a D^{\otimes f}\right) / \operatorname{Tr}(D)^{f} \text { for } a \in M_{n+1}^{\otimes f} .
$$

Observe that $\psi(1)=1$ and that for $a \in M_{n+1}^{\otimes f-1} \psi(a)$, defined for the $(f-1)^{\text {th }}$ tensor power coincides with $\psi(a \otimes 1)$ defined for the $f^{\text {th }}$ tensor power. Hence $\psi$ can be extended to the inductive limit of the $M_{n+1}^{\otimes f}$ 's.

To compute the weight vector of $\psi_{\mid A(t)}$, we assume for the moment that the eigenvalues $\alpha_{j}$ of $D$ are of absolute value 1 . Let $\alpha_{j}=\beta_{j}+i \gamma_{j}$. Then it is easy to see that $D_{\text {|span }\left\{\vec{w}_{j}, \vec{w}_{-j}\right\}}$ with respect to this basis is given by the orthogonal matrix

$$
\left(\begin{array}{cc}
\beta_{j} & \gamma_{j} \\
-\gamma_{j} & \beta_{j}
\end{array}\right)
$$

By the previous lemma it is enough to compute the weight vector for $A(1)$ which is equal to the centralizer of $\varrho^{\otimes f}(O(n+1))^{\prime}$. So if $p$ is a minimal idempotent of $A(1)$, we obtain an irreducible representation of $O(n+1)$ on $p V^{\otimes f}$. If $\chi^{(\lambda)}$ is the corresponding character, one has

$$
\psi(p)=\operatorname{Tr}\left(D^{\otimes f} p\right) / \operatorname{Tr}(D)^{f}=\chi^{(\lambda)}(D) /(\operatorname{Tr}(D))^{f} .
$$

It is well-known that the character formulas are rational functions in the eigenvalues of $D$. Hence the formula also holds in general for arbitrary $\alpha_{j}$ 's by analytic continuation (evaluating the functional $\psi$ only involves rational operations).

So it follows from our discussion and the previous lemma

Corollary 5.3. (a) Let $A$ and $\psi$ be as above. Then $A$ is semisimple for all but finitely many values of $t$ and $\psi$ is a trace on it for all values of $t$. If $A$ is semisimple, the weight vector is given by

$$
\left(\chi^{(\lambda)}(D) /(\operatorname{Tr}(D))^{f}\right)_{\lambda \in \Gamma_{f}},
$$

where $\chi^{(\lambda)}(D)$ is the character corresponding to $\lambda$ of an orthogonal matrix with the same eigenvalues as $D$. If there is no orthogonal matrix with these eigenvalues we take the unique analytic extension of the character formula to these values. 
(b) In general, the image under $\pi_{\psi}$ of any subalgebra of the centralizer algebra of the $f^{\text {th }}$ tensor power of the standard representation of $U_{q} s o_{n+1}$ is semisimple.

To compute the weights of the structure trace of $C_{f}(r, q)$ we will compute the weight vector of $\psi$ given by a special $D$ namely for

$$
D=D(q)=\operatorname{diag}\left(q^{i}, i \in I\right) .
$$

( $D$ can be characterized as the $q$ exponential of the matrix corresponding to the sum of all positive roots in $G$ with respect to a (up to scalar multiples unique) invariant bilinear form). Observe that for $x$ as defined in (5.2) one has $x=\operatorname{Tr}(D)=\sum_{i \in I} q^{i}$. It
follows from a straightforward computation that

$$
\psi(R)=q^{n} / x \quad \text { and } \quad \psi\left(R^{-1}\right)=q^{-n} / x .
$$

Lemma 5.4. Let $A_{f}(t)=\Phi\left(C_{f}\left(t^{n}, t\right)\right)$ (where $t$ is a variable) and let $\psi$ be as defined above by the density matrix $D=D(q)$.

If $t=q, \psi \circ \Phi$ coincides with the structure trace $\operatorname{tr}$ on $C_{f}\left(q^{n}, q\right)$.

Proof. It follows from the previous corollary that $\psi$ is a trace on $A(t)$. Now observe that $\psi\left(\left(1 \otimes e_{i j}\right) R^{ \pm 1}\right)=\psi\left(e_{i j}\right) \psi\left(R^{ \pm 1}\right)$. By linearity, it follows from this

$$
\psi\left((1 \otimes a) R_{1}^{ \pm 1}\right)=\psi(1 \otimes a) \psi\left(R_{1}^{ \pm 1}\right)
$$

for any $a \in M_{n}(\mathbf{C})^{\otimes f-1}$. So, in particular, $\psi\left(\Phi\left(a g_{1}\right)\right)=\psi(\Phi(a)) \psi\left(\Phi\left(g_{1}\right)\right)$ for all $a \in C_{2, f}\left(t^{n}, t\right)$. Applying the inner automorphism $\eta_{f}$ (see defin. of braid groups in Sect. 2), it follows from this and (5.7) that $\psi \circ \Phi$ satisfies the properties of the functional $\mathrm{tr}$ in Lemma $3.4(\mathrm{~d})$. Hence $\psi \circ \Phi=\operatorname{tr}$ by that lemma.

We are now going to compute the rational functions $Q_{\lambda}$ of Theorem 3.6. The idea is very simple. By the last proposition one can compute $Q_{\lambda}\left(q^{n}, q\right)$, which is nothing else than the orthogonal character $\chi^{(\lambda)}(D)$ belonging to the diagram $\lambda$ for the special choice of $D$ as in (5.6). So now the only work consists of rewriting these character formulas in such a way that after replacing certain occurrences of $q^{n}$ by $r$, one obtains a rational function in $q$ and $r$ which no longer depends on $n$. This will result in transforming the orthogonal (and symplectic) character formulas (in a special case) from "root form" into "hook length form" (this terminology is due to MacDonald).

Recall that for an orthogonal matrix $D$ with eigenvalues 1 and $z_{j}^{2}, z_{j}^{-2}$, $j=1,2, \ldots k$, the character $\chi^{(\lambda)}$ is determined by

$$
\chi^{(\lambda)}(D)=\frac{\operatorname{det}\left(z_{j}^{\lambda_{i}+k+1-2 i}-z_{j}^{-\lambda_{i}-k-1+2 i}\right)}{\operatorname{det}\left(z_{j}^{k+1-2 i}-z_{j}^{-k-1+2 i}\right)} .
$$

By Weyl's character formula for the odd dimensional group the denominator $\Delta= \pm \operatorname{det}\left(z_{j}^{2 i-1}-z_{j}^{-2 i+1}\right)_{i j}$ can be expressed by the product

$$
\Delta=\prod_{i=1}^{k} z_{i}-z_{i}^{-1} \prod_{1 \leqq i<j \leqq k}\left(z_{i} z_{j}-\left(z_{i} z_{j}\right)^{-1}\right)\left(z_{i} z_{j}^{-1}-z_{i}^{-1} z_{j}\right) .
$$

For more convenient notation, we use the symbol $[s]_{q}$ to denote $q^{s}-q^{-s}$ and $[y+s]_{q}$ for $r q^{s}-r^{-1} q^{-s}$. Let $\lambda=\left[\lambda_{1}, \lambda_{2}, \ldots \lambda_{k}\right]$ and let $z_{i}=q^{\lambda_{i}+k-i+1 / 2}$. Then it follows from the last 2 formulas for $D=\operatorname{diag}\left\{q^{i}, i \in I\right\}$, 


$$
\begin{aligned}
\chi^{(\lambda)}(D) & =\frac{\Delta\left(z_{1}, \ldots z_{k}\right)}{\Delta\left(q^{1 / 2}, q^{3 / 2}, \ldots q^{(2 k-1) / 2}\right)} \\
& =\prod_{s=1}^{k} \frac{\left[(2 k+1) / 2+\lambda_{s}-s\right]_{q}}{[(2 k+1) / 2-s]_{q}} \prod_{1 \leqq s<t \leqq k} \frac{\left[2 k+1+\lambda_{s}+\lambda_{t}-t-s\right]_{q}\left[\lambda_{s}-\lambda_{t}+t-s\right]_{q}}{[2 k+1-t-s]_{q}[t-s]_{q}} .
\end{aligned}
$$

Let $\lambda$ be a Young diagram and let $(i, j)$ denote the $j^{\text {th }}$ box in the $i^{\text {th }}$ row. Let $\lambda_{i}$ and $\lambda_{j}^{\prime}$ be the number of boxes in the $i^{\text {th }}$ row and $j^{\text {th }}$ column respectively. The hooklength $h(i, j)$ is defined by $h(i, j)=\lambda_{i}-i+\lambda_{j}^{\prime}-j+1$. We shall need the following observation (see [Mc, Example 1 on p. 27]). Let $\lambda$ be a diagram with $m$ rows. Then we have for $n>m$ (with $\lambda_{m+1}=\lambda_{m+2}=\ldots=\lambda_{n}=0$ )

$$
\prod_{1 \leqq s<t \leqq n} \frac{\left[\lambda_{s}-\lambda_{t}+t-s\right]_{q}}{[t-s]_{q}}=\prod_{(i, j) \in \lambda} \frac{[n+j-i]_{q}}{[h(i, j)]_{q}} .
$$

In particular, if we replace $q^{n}$ by the variable $r$, we obtain a rational function in $q$ and $r$ which is independent of $n$. Moreover we shall also need the observation (already known to Frobenius (see $[\mathrm{EK}]))$ that the sets $\left\{\lambda_{j}-j, j=1,2, \ldots \lambda_{1}^{\prime}\right\}$ and $\left\{i-1-\lambda_{i}^{\prime}, i\right.$ $\left.=1,2, \ldots \lambda_{1}\right\}$ contain exactly $\lambda_{1}^{\prime}$ respectively $\lambda_{1}$ elements and are mutually disjoint. Hence their union coincides with $\left\{-\lambda_{1}^{\prime},-\lambda_{1}^{\prime}+1, \ldots \lambda_{1}-1,\right\}$. In particular, we have

$$
\{1,2, \ldots m-1\}=\mathbf{R}^{+} \cap\left(\left\{\lambda_{j}-j, j=1,2, \ldots \lambda_{1}^{\prime}\right\} \cup\left\{i-1-\lambda_{i}^{\prime}, i=1,2, \ldots \lambda_{1}\right\}\right) .
$$

This was used by El Samra and King to define for each Young diagram $\lambda$ a polynomial $P_{\lambda}$ which, evaluated at $n$ gives the dimension of the irreducible representation of $O(n)$ belonging to $\lambda . P_{\lambda}$ can be written as

$$
P_{\lambda}(x)=\prod_{(i, j) \in \lambda} \frac{x-1+d(i, j)}{h(i, j)},
$$

with the product taken over all pairs $(i, j)$ specifying row and column of a box of $\lambda$. The quantities $d(i, j)$ are defined by

$$
d(i, j)= \begin{cases}\lambda_{i}+\lambda_{j}-i-j+1 & \text { if } i \leqq j \\ -\lambda_{i}^{\prime}-\lambda_{j}^{\prime}+i+j-1 & \text { if } i>j .\end{cases}
$$

We see immediately that

and

$$
-2 \lambda_{1}^{\prime}+1 \leqq d(i, j) \leqq 2 \lambda_{1}-1 \text { for all }(i, j) \in \lambda
$$

$$
-2 \lambda_{1}^{\prime}+1 \leqq-2 \lambda_{j}^{\prime}+2 j-1 \leqq 2 \lambda_{1}-1
$$

As already mentioned before, these polynomials give us the dimensions of irreducible representations of $O(n)$. In particular, it follows from Weyl's dimension formula for $n=2 k+1$,

$$
P_{\lambda}(2 k+1)=\prod_{i=1}^{k} \frac{(2 k+1) / 2+\lambda_{i}-i}{(2 k+1) / 2-i} \prod_{1 \leqq i<j \leqq k} \frac{\left(2 k+1+\lambda_{i}+\lambda_{j}-i-j\right)\left(\lambda_{i}-\lambda_{j}+j-i\right)}{(2 k+1-i-j)(j-i)} .
$$


We shall need this to prove the following

Theorem 5.5. Let $\lambda$ be a Young diagram with $m$ rows and let $Q_{\lambda}(r, q)$ be the rational function given by

$$
Q_{\lambda}(r, q)=\prod_{(j, j) \in \lambda} \frac{\left[y+\lambda_{j}-\lambda_{j}^{\prime}\right]_{q}+[h(j, j)]_{q}}{[h(j, j)]_{q}} \prod_{(i, j) \in \lambda, i \neq j} \frac{[y+d(i, j)]_{q}}{[h(i, j)]_{q}} .
$$

Then we have for all $k>m$ and $D$ the $(2 k+1) \times(2 k+1)$ matrix as in the previous corollary,

$$
Q_{\lambda}\left(q^{2 k}, q\right)=\chi^{(\lambda)}(D)
$$

In particular, $Q_{\lambda}$ coincides with the rational function in Theorem (3.6).

Proof. Using the notation introduced before (5.8) we define the rational function $Q_{\lambda}^{(k)}$ by

$$
\begin{aligned}
Q_{\lambda}^{(k)}= & \prod_{j=1}^{m} \frac{\left[y / 2+\lambda_{j}-j+1 / 2\right]_{q}}{[y / 2-j+1 / 2]_{q}} \prod_{1 \leqq r<s \leqq k} \frac{\left[y+\lambda_{r}+\lambda_{s}-r-s\right]_{q}}{[y-r-s]_{q}} \\
& \times \prod_{(i, j) \in \lambda} \frac{[y-k-1+j-i]_{q}}{[h(i, j)]_{q}}
\end{aligned}
$$

By (5.8) and (5.9) we have

$$
Q_{\lambda}^{(k)}\left(q^{2 k}, q\right)=\psi^{(\lambda)}(D) .
$$

We are going to show by induction that $Q_{\lambda}^{(k)}$ does not depend on $k$, i.e. one obtains the same rational function for any $k, k>m$. It follows from the definitions that

$$
\frac{Q_{\lambda}^{(k+1)}}{Q_{\lambda}^{(k)}}=\prod_{r=1}^{k} \frac{\left[y+\lambda_{r}-r-k-1\right]_{q}}{[y-r-k-1]_{q}} \prod_{(i, j) \in \lambda} \frac{[y-k-2+j-i]_{q}}{[y-k-1+j-i]_{q}} .
$$

Evaluating the second product on the right-hand side separately for each row of $\lambda$, one checks easily that the right-hand side is equal to 1.

By the same procedure, one defines rational functions in the variable $y$ by replacing $[n]_{q}$ and $[y+n]_{q}$ by $n$ and $y+n$ respectively. For $q=1$ the character of $D$ is equal to the dimension of the representation belonging to $\lambda$. Hence the rational function obtained is the polynomial $P_{\lambda}(y)$. In particular, as linear terms are obviously prime elements in a polynomial ring, the factors in the denominator, which contain a $y$, have to cancel with factors in the numerator. By carrying out the same cancellations for the corresponding $[y+n]_{q}$ 's, one obtains

$$
\begin{aligned}
\chi^{(\lambda)}(D)= & \prod_{j=1}^{m} \frac{q^{k-j+1 / 2}+q^{-k+j-1 / 2}}{q^{\lambda_{j}+k-j+1 / 2}+q^{-\lambda_{j}-k+j-1 / 2}} \\
& \times \prod_{j=1}^{m} \frac{\left[2 k+1+2 \lambda_{j}-2 j\right]_{q}}{[2 k+1-2 j]_{q}} \\
& \times \prod_{1 \leqq r<s \leqq k} \frac{\left[2 k+1+\lambda_{r}+\lambda_{s}-r-s\right]_{q}\left[\lambda_{r}-\lambda_{s}+s-r\right]_{q}}{[2 k+1-r-s]_{q}[s-r]_{q}} \\
= & \prod_{j=1}^{m} \frac{r^{1 / 2} q^{-j+1 / 2}+r^{-1 / 2} q^{j-1 / 2}}{r^{1 / 2} q^{\lambda_{j}-j+1 / 2}+r^{-1 / 2} q^{-\lambda_{j}+j-1 / 2}} \prod_{(i, j) \in \lambda} \frac{[y+d(i, j)]_{q}}{[h(i, j)]_{q}} .
\end{aligned}
$$


If $\lambda_{j} \geqq j,(j, j) \in \lambda$ and

$$
\frac{[y+d(j, j)]_{q}}{r^{1 / 2} q^{\lambda_{j}-j+1 / 2}+r^{-1 / 2} q^{j-\lambda_{j}-1 / 2}}=r^{1 / 2} q^{\lambda_{j}-j+1 / 2}-r^{-1 / 2} q^{j-\lambda_{j}-1 / 2} .
$$

If $\lambda_{j}<j$, we have $-m<\lambda_{j}-j<0$. Hence the denominator in the first product of $(*)$ belonging to such a $j$ cancels with a factor in the numerator of that product. By Frobenius' observation, the exponents of the remaining factors are given by the set $\left\{j-1-\lambda_{j}^{\prime}+1 / 2,(j, j) \in \lambda\right\}$. Multiplying the factors belonging to the same $j$, one sees that $Q_{\lambda}(r, q)$ can be written as in the statement.

By Lemma 5.4 and the uniqueness of the structure trace, the function $Q_{\lambda}(r, q)$ coincides with the rational function in Theorem 3.6 denoted by the same symbol for $r=q^{2 k}, k=1,2, \ldots$. This means that for each arbitrary fixed $q$ which is not a root of unity, they coincide at infinitely many different values of $r$. Hence they have to be the same for arbitrary values of $r$, which proves the last statement.

Corollary 5.6. (a) If $r \neq \pm q^{n}$ for any $n \in \mathbf{Z}$ and $q$ is not a root of unity, the structure trace $\operatorname{tr}$ is faithful on $C_{f}(r, q)$ for all $f \in \mathbf{N}$. In particular $C_{f}(r, q)$ is semisimple with the same decomposition into full matrix rings as $C_{f}$ for all $f \in \mathbf{N}$.

(b) Let $q \in \mathbf{C}$ be arbitrary. Then

(b1) $\Gamma\left(q^{-1}, q\right)$ is not defined.

(b2) $\Gamma(1, q)=\{[0],[1]\}$ and $Q_{[0]}(1, q)=Q_{[1]}(1, q)=1$.

(b3) $\Gamma(q, q)=\{[m], m \in \mathbf{N}\} \cup\{[0]\} \cup\left\{\left[1^{2}\right]\right\}, Q_{[1]}(q, q)=Q_{\left[1^{2}\right]}(q, q)=1$ and $Q_{[m]}(q, q)$ $=2$ for $m>1$.

(c) If $r=q^{n}$ and $q$ is not a root of unity, $\Gamma(r, q)$ consists of all Young diagrams $\lambda$ for which

(c1) $\tilde{\lambda}_{1}+\widetilde{\lambda}^{2}<n+1$ for $n>0$,

(c2) $2 \lambda_{1}<|n|+2$ for $n<0, n$ even,

(c3) $\lambda_{1}+\lambda_{2}<|n|+3$ for $n<0, n$ odd.

(d) If $|q|=1$ and $q$ is not a root of unity, $\pi_{\mathrm{tr}}$ can only be a $\mathrm{C}^{*}$ representation of $C_{\infty}(r, q)$ if one of the conditions of $(\mathrm{b})$ is satisfied. In particular, there can only be unitary representations of $C_{\infty}(r, q)$ if $q$ is a root of unity.

(e) $Q_{\lambda}\left(r^{-1}, q^{-1}\right)=Q_{\lambda}(r, q)=Q_{\lambda}(-r,-q)$ and $Q_{\lambda}\left(r, q^{-1}\right)=Q_{\lambda^{\prime}}(r, q)$.

Proof. Obviously, $Q_{\lambda}(r, q)=0$ only if one of the factors in the expression of the last theorem is equal to 0 . This can only happen if $r= \pm q^{n}$ for some $n \in \mathbf{Z}$. So in all the other cases $\operatorname{tr}$ is faithful on $C_{f}(r, q)$ for all $f \in \mathbf{Z}$. The claim now follows from Theorem 2.8 .

(b) and (c) are straightforward computations (see also [W-2, Corollary 3.5]). In particular, it follows from (c) and (a) that $\Gamma(r, q)$ is never finite if $q$ is not a root of unity. Hence (d) follows from this and Corollary 2.11 .

(e) can be checked directly from the definition of $Q_{\lambda}$.

\section{Special Semisimple and Unitary Quotients of $C_{f}(r, q)$}

In this section, we determine the structure of $\pi_{\mathrm{tr}}\left(C_{f}\left(q^{n}, q\right)\right.$ with $n \in \mathbf{Z}$ and $q$ a root of unity. The fact that the image is semisimple has already been shown for even $n>1$ using solutions of the QYBE coming from quantum groups of type $B$ (see Lemmas 5.2-5.4). The same proof can be extended to the general case using the solutions for types $C$ and $D$ (which would cover the odd integers $\neq-1$ ) and isomorphisms as 
defined in Proposition 3.2 which we will omit. Having the explicit formulas for the $Q_{\lambda}$ 's available, it is also possible to check semisimplicity directly by Lemma 1.4 which, however, is somewhat messy and does not provide any insight.

To determine the explicit structure of $\pi_{\mathrm{tr}}\left(C_{f}\left(q^{n}, q\right)\right)$, we will compute $\tilde{\Gamma}(r, q)$, as defined before Lemma 4.2 and check whether it satisfies the conditions of Theorem 4.4. This involves somewhat tedious computations. Some simplifications can be made if one rewrites the formulas for $Q_{\lambda}\left(q^{n}, q\right)$ as character formulas in root form, thereby reversing the process in the proof of Theorem 5.5. This also explains why one obtains the conditions for $\Gamma(r, q)$ in terms of highest roots (see Theorem 6.4). Our initial intention was, however, to classify all unitary braid representations factoring through $C_{\infty}(r, q)$. This and also some subtleties with the root lattice and various isomorphisms between different cases are the reasons why we left the presentation in the original way.

We first do the case $r=q^{2}$ with $q$ a primitive $l^{\text {th }}$ root of unity which is slightly different. It nevertheless also shows the general pattern of our strategy for the other cases. First observe that by Theorem 5.5, $Q_{\lambda}\left(q^{2}, q\right)=\chi^{(\lambda)}(D)$, where $D$ is an orthogonal matrix with eigenvalues $q^{-1}, 1$ and $q$ and $\chi^{(\lambda)}$ is its character belonging to the representation labelled by the Young diagram $\lambda$ (see [Wy]). It follows from Weyl's character formulas that

and

$$
Q_{\lambda}\left(q^{2}, q\right)=0 \quad \text { if } \quad \lambda_{1}^{\prime}+\lambda_{2}^{\prime} \geqq 4
$$

$$
Q_{[m, 1]}\left(q^{2}, q\right)=Q_{[m]}\left(q^{2}, q\right)=\frac{q^{m+1 / 2}-q^{-m-1 / 2}}{q^{1 / 2}-q^{-1 / 2}} .
$$

The following statements are almost immediate consequences from these formulas :

(1) If $l=2 k+1$ is odd, $Q_{[k]}\left(q^{2}, q\right)=0$ and if $q=e^{2 \pi i / l}, \quad Q_{[m]}\left(q^{2}, q\right)$ $=\sin ((2 m+1) \pi / l) / \sin (\pi / l)>0$ for $m=1,2, \ldots k-1$.

(2) If $l=2 k$ is even, $Q_{[m]}\left(q^{2}, q\right) \neq 0$ for all $m \in \mathbf{N}$. If $q=e^{\pi i / k}$, one has $Q_{[m]}\left(q^{2}, q\right)>0$ for $m<k$ and $Q_{[k]}\left(q^{2}, q\right)=-Q_{[k-1]}\left(q^{2}, q\right)=-Q_{[k-1,1]}\left(q^{2}, q\right)$.

Proposition 6.1. Let $q$ be a primitive $l^{\text {th }}$ root of unity and let $r=q^{2}$. Then

(a) $\pi_{\mathrm{tr}}\left(C_{f}(r, q)\right)$ is semisimple for all $f \in \mathbf{N}$. Let $k$ be the largest integer $\leqq l / 2$. The graph $\Gamma\left(q^{2}, q\right)$ is given by

$$
\Gamma\left(q^{2}, q\right)=\{[0]\} \cup\{[m],[m, 1], 0 \leqq m \leqq k-1\} \cup\left\{\left[1^{3}\right]\right\},
$$

where the edges are given by the Young lattice with the only exception that for l even the diagrams $[k-1]$ and $[k-1,1]$ are not connected.

(b) $\pi_{\mathrm{tr}}$ is a $\mathrm{C}^{*}$ representation of $C_{\infty}\left(q^{2}, q\right)$ if and only if $q= \pm e^{ \pm 2 \pi i / l}$.

Proof. As already mentioned, the semisimplicity follows from Lemmas 5.2-5.4. In the odd case (i.e. $l$ is odd) also $q^{2}$ is a primitive $l^{\text {th }}$ root of unity. Hence all diagrams of the form $[m]$ or $[m, 1]$ with $m<k$ are $l$ diagrams. Moreover, by (1) $Q_{\lambda}\left(q^{2}, q\right)=0$ for all diagrams $\lambda$ which are adjacent to $\Gamma(r, q)$. This shows the claim in the odd case by Lemma 4.2.

The even case is slightly more complicated. Here $q^{2}$ is a primitive $k^{\text {th }}$ root of unity. The structure of $C_{f}\left(q^{2}, q\right)$ is determined by Lemma 4.1 for $f=1,2, \ldots k-1$ with $\varrho_{f} \cong \pi_{[f-1,1]} \oplus \varrho[\mathrm{f}]$ for $f>1$. By the restriction rule (see Lemma 4.1) and Proposition 2.2 the only possible representations which can appear in $\varrho_{k}$ are $\pi_{[k]}^{(2, k)}$ 
and $\pi_{[k-1,1]}^{(2, k)}$. As $Q_{\left[k-2,1^{2}\right]}\left(q^{2}, 2\right)=0$, it follows from Theorem 4.4 and (2) that

$$
\begin{gathered}
\bar{Q}_{[k-1,1]}\left(q^{2}, q\right)=Q_{[k-1,1]}\left(q^{2}, q\right) \neq 0, \\
\bar{Q}_{[k]}\left(q^{2}, q\right)=Q_{[k-1,1]}\left(q^{2}, q\right)+Q_{[k]}\left(q^{2}, q\right)=0 .
\end{gathered}
$$

Hence $\pi_{\mathrm{tr}}\left(C_{k}\left(q^{2}, q\right)\right)$ is semisimple and its Hecke algebra part only consists of one simple component which is isomorphic to $\pi_{[k-1,1]}^{(2, k)}\left(H_{k}\left(q^{2}\right)\right)$. This shows the claim for $f=k$. For $f>k$, just observe that there is no semisimple representation of $H_{k+1}\left(q^{2}\right)$ whose restriction to $H_{k}\left(q^{2}\right)$ would just consist of $\pi_{[k-1,1]}^{(2, k)}$ (see Proposition 2.2).

The rest of our structure analysis follows this pattern, i.e. it consists of checking for which $\lambda$ 's $Q_{\lambda}\left(q^{n}, q\right)$ or respectively $Q_{\lambda}\left(q^{n}, q\right)$ are zero. The following lemmas will reduce the number of different cases for $\Gamma\left(q^{n}, q\right)$.

Lemma 6.2. Let $q$ be a root of unity and let $r=q^{n}, n \in \mathbf{Z}$. Using the isomorphisms of Proposition 3.2, one can assume without restriction of generality

(a) $q$ is a primitive $2 l^{\text {th }}$ root of unity, $l \in \mathbf{N}$,

(b) $r=q^{n}$ with $|n|<l$ and

(c) either both $l$ and $n$ are even with $n>0$ or $l-n$ is odd.

Proof. Let $q$ be a primitive $l^{\text {th }}$ root of unity with $l$ odd and let $\varepsilon(n)=(-1)^{n-1}$. Then, by Proposition 3.2 (c),

$$
C_{f}\left(q^{n}, q\right) \cong C_{f}\left(-q^{n},-q\right)=C_{f}\left(\varepsilon(n)(-q)^{n},-q\right)=C_{f}\left((-q)^{\varepsilon(n) n},-q\right) .
$$

Observe that $-q$ is a primitive $2 l^{\text {th }}$ root of unity. It is now easy to check that either $r=q^{\varepsilon(n) n}=-1$ or $\varepsilon(n) n$ can be replaced by an integer of absolute value less than $l$ without changing the value of $r$.

If both $l$ and $n$ are even and $n<0$, one has

$$
C_{f}\left(q^{-n}, q\right) \cong C_{f}\left(-q^{l-n}, q\right) \cong C_{f}\left(q^{l-n},-q\right)=C_{f}\left((-q)^{l-n},-q\right)
$$

with $0<l-n<l$ even. Hence one can assume $n>0$ in this case. By the same manipulations one can reduce the case with both $l$ and $n$ odd to the case $l$ odd and $n$ even.

We will also need the following observations:

Let $r=q^{n}$. Then the numerator of the factor of $Q_{\lambda}$ belonging to the box $(i, j) \in \lambda$ is equal to 0 if

(a) $q^{2(n+d(i, j))}=1$ if $(i, j)$ is an off-diagonal box (i.e. if $\left.i \neq j\right)$,

(b) $q^{n-2 \lambda_{j}+2 j-1}=-1$ or $q^{n+2 \lambda_{j}-2 j+1}=1$ if $i=j$, i.e. for diagonal boxes $(j, j) \in \lambda$.

Lemma 6.3. (a) Let $q$ be a primitive $2 l^{\text {th }}$ root of unity and let $n \in \mathbf{Z},|n|<l$. Let $N^{\text {od }}$ be the product of the numerators of the factors of $Q_{\lambda}\left(q^{n}, q\right)$ belonging to off diagonal boxes of $\lambda$. Then $N^{\text {od }}$ is equal to 0 if

$$
\begin{array}{lll}
\lambda_{1}+\lambda_{2}=l-n+2 & \text { or } \quad \lambda_{1}^{\prime}+\lambda_{2}^{\prime}=n+2 \text { for } n>2, \\
\lambda_{1}+\lambda_{2}=2-n \quad \text { or } \quad \lambda_{1}^{\prime}+\lambda_{2}^{\prime}=l+n+2 \text { for } n<-1,
\end{array}
$$

$N^{\text {od }}$ is not equal to 0 if

$$
\begin{gathered}
\lambda_{1}+\lambda_{2} \leqq l-n+1 \text { and } \lambda_{1}^{\prime}+\lambda_{2}^{\prime}=n+1 \text { for } n>2, \\
\lambda_{1}+\lambda_{2} \leqq 1-n \text { and } \lambda_{1}^{\prime}+\lambda_{2}^{\prime} \leqq l+n+1 \text { for } n<-1 .
\end{gathered}
$$


(b) Let $N^{d}$ be the product of the numerators of the factors of $Q_{\lambda}\left(q^{n}, q\right)$ belonging to diagonal boxes of $\lambda$. If $n$ is even and $l$ is odd, $N^{d}$ is equal to 0 if

$$
\lambda_{1}^{\prime}=(l+n+1) / 2 .
$$

If $n$ is odd and $l$ is even, $N^{d}$ is equal to 0 if

$$
\begin{aligned}
& \lambda_{1}=(2 l+1-n) / 2 \quad \text { or } \quad \lambda_{1}^{\prime}=(l+n+1) / 2 \quad \text { if } n>2, \\
& \lambda_{1}=(1-n) / 2 \quad \text { or } \quad \lambda_{1}^{\prime}=(l+n+1) / 2 \quad \text { if } n<-1,
\end{aligned}
$$

$N^{d}$ is never equal to 0 if both $n$ and l are even. If $n$ is even and $l$ is odd, $N^{d}$ is not equal to 0 if

$$
\lambda_{1}^{\prime} \leqq(l+n-1) / 2 .
$$

If $n$ is odd and $l$ is even, $N^{d}$ is not equal to 0 if

$$
\begin{aligned}
& \lambda_{1} \leqq(2 l-1-n) / 2 \quad \text { and } \lambda_{1}^{\prime} \leqq(l+n-1) / 2 \text { if } n>2, \\
& \lambda_{1} \leqq(-1-n) / 2 \text { and } \lambda_{1}^{\prime}=(l+n-1) / 2 \text { if } n<-1 .
\end{aligned}
$$

Proof. The proof consists of checking when the conditions stated before this lemma hold. In case (a), one observe that

$$
-\lambda_{1}^{\prime}-\lambda_{2}^{\prime}+2=f(2,1) \leqq d(i, j) \leqq d(1,2)=\lambda_{1}+\lambda_{2}-2 \text { for all }(i, j) \in \lambda, i \neq j .
$$

This is also true if $\lambda=[m]$, i.e. it only consists of one row. One just notes that in this case $d(2,1)=m-2=d(1, m)$. The case with only one column goes similarly.

In case (b) one observes similarly that

and

$$
-2 \lambda_{i}^{\prime}+1 \leqq 2 \lambda_{j}-2 j+1 \leqq 2 \lambda_{1}-1
$$

$$
-2 \lambda_{1}^{\prime}+1 \leqq-2 \lambda_{j}^{\prime}+2 j-1 \leqq 2 \lambda_{1}-1
$$

Moreover, one also uses the fact that if $n$ is even both $n-2 \lambda_{j}^{\prime}+2 j-1$ and $n+2 \lambda_{j}-2 j+1$ are odd. But as $l$ is even, any odd power of $q$ can never be equal to 1 or -1 .

To determine the semisimple quotients of $C_{f}(r, q)$ which factor over $\operatorname{tr}$ in general, we need the following facts:

Recall that for $n>2$ the functions $Q_{\lambda}\left(q^{n}, q\right)$ are characters of an orthogonal $(n+1) \times(n+1)$ matrix $D$ with eigenvalues $\left\{q^{ \pm(2 i-1)}, i=1,2, \ldots n / 2\right\}$ for $n$ even and $\left\{q^{ \pm i}, i=0,1, \ldots(n+1) / 2\right\}$ for $n$ odd (where $q^{0}=1$ appears twice). This was shown in the first case for the odd dimensional orthogonal groups in Theorem 5.5. It can be done in a similar fashion for the even dimensional orthogonal case. Also observe that in both cases one has $\operatorname{det}(D)=1$. It follows from Weyl's character formulas that (a) $Q_{\lambda}\left(q^{n}, q\right)=0$ if $\lambda_{1}^{\prime}+\lambda_{2}^{\prime} \geqq n+2$.

(b) If $\lambda_{1}^{\prime} \geqq(n+2) / 2$ let $\bar{\lambda}$ be the diagram which has the same number of boxes as $\lambda$ in all columns except the first one, which now contains $n+1-\lambda_{1}^{\prime}$ boxes. By (a) $\bar{\lambda}$ is a Young diagram if $Q_{\lambda}\left(q^{n}, q\right) \neq 0$. Then one has

$$
Q_{\lambda}\left(q^{n}, q\right)=Q_{\bar{\lambda}}\left(q^{n}, q\right) .
$$

We can now give an explicit description of the structure of $\pi_{\mathrm{tr}}\left(C_{f}\left(q^{n}, q\right)\right)$ if $q$ is a root of unity and $n$ is an integer by computing the corresponding graphs $\Gamma\left(q^{n}, q\right)$. An example how to get from the graph to the algebra has already been given after Theorem 4.4 
Theorem 6.4. Let $q$ be a root of unity and let $r=q^{n}, n \in \mathbf{Z} \backslash\{-1\}$. Then $\pi_{\mathrm{tr}}\left(C_{f}\left(q^{n}, q\right)\right)$ $\left(\cong \pi_{t r}\left(C_{f}\left(-q^{-n}, q\right)\right)\right)$ is semisimple for all $f \in \mathbf{N}$ and $\Gamma\left(q^{n}, q\right)$ is a subgraph of the Young lattice except in the case treated in Proposition 6.1 with l even. There are the following cases left, which are not already covered by Corollary 5.6 and Proposition 6.1:

(a) If $q$ is a primitive $2 l^{\text {th }}$ root of unity and $2<n<l-2$,

$$
\Gamma\left(q^{n}, q\right)=\left\{\lambda, \lambda_{1}+\lambda_{2} \leqq l-n+1 \text { and } \lambda_{1}^{\prime}+\lambda_{2}^{\prime} \leqq n+1\right\} \cup\left\{\left[l-n+1,1^{n-1}\right]\right\} .
$$

(b) If $n<-1$, even and $l$ is odd,

$$
\Gamma\left(q^{n}, q\right)=\left\{\lambda, \lambda_{1}+\lambda_{2} \leqq-1-n \text { and } \lambda_{1}^{\prime} \leqq(l+n-1) / 2\right\} .
$$

(c) If $n<-1$, odd and $l$ is even,

$$
\Gamma\left(q^{n}, q\right)=\left\{\lambda, \lambda_{1} \leqq(-1-n) / 2 \text { and } \lambda_{1}^{\prime} \leqq(l+n-1) / 2\right\} .
$$

By the previous lemmas all other cases of $\Gamma\left(q^{n}, q\right)$ with $q$ a root of unity can be reduced to either one of the cases listed above or to one of the cases in Corollary 5.6 (b) or Proposition 6.1.

In all these cases, $\pi_{\mathrm{tr}}$ defines a unitary representation of $B_{\infty}$ if $q=e^{\pi i / l}$.

Proof. The cases $r=1, r=q$ and $r=q^{2}$ have already been treated. By Lemma 6.2 one can assume $q$ to be a primitive $2 l^{\text {th }}$ root of unity. As already mentioned at the beginning of the section, $\pi_{\mathrm{tr}}\left(C_{f}\left(q^{n}, q\right)\right)$ is semisimple for all $f \in \mathbf{N}$.

Let $2<n<l-2$. Observe that $\lambda_{1}^{\prime}+\lambda_{2}^{\prime} \leqq n+2$ and $\lambda_{1}+\lambda_{2} \leqq l-n+2$ imply in particular $\lambda_{1}^{\prime} \leqq(l+n-1) / 2$ and $\lambda_{1} \leqq l-(n+1) / 2$. Hence, by Lemma $6.2, Q_{\lambda}\left(q^{n}, q\right) \neq 0$ if $\lambda_{1}^{\prime}+\lambda_{2}^{\prime} \leqq n+1$ and $\lambda_{1}+\lambda_{2} \leqq l-n+1$. Moreover, if $\lambda_{1}^{\prime}+\lambda_{2}^{\prime}=n+2$ or $\lambda_{1}+\lambda_{2}$ $=l-n+2$, the numerator of $Q_{\lambda}\left(q^{n}, q\right)$ is equal to 0 . In this case, one checks that $h(i, j)<l$ for all $(i, j) \in \lambda$ except if $\lambda=\left[l-n+1,1^{n-1}\right]$. So except for this special case one has $Q_{\lambda}\left(q^{n}, q\right)=0$ and by remark (b) $Q_{\left[k-n+1,1^{n-1}\right]}\left(q^{n}, q\right)=Q_{[k-n+1]}\left(q^{n}, q\right)$ is welldefined and nonzero.

It follows from the considerations above that the only hook diagram $\lambda$ with $l-1$ boxes for which $Q_{\lambda}\left(q^{n}, q\right) \neq 0$ is $\lambda=\left[l-n, 1^{n-1}\right]$. If $\lambda$ is a diagram with less than $n-2$ boxes in the first 2 columns and less than $l-n+2$ boxes in the first 2 rows, it follows again by Lemma 6.3 that $Q_{\lambda}\left(q^{n}, q\right) \neq 0$.

By Lemma 6.2 the only remaining cases (up to isomorphism) are $n<-1$ even and $l$ odd, and $n<-1$ odd and $l$ even. One computes $\Gamma\left(q^{n}, q\right)$ as above using Lemma 6.3 (in these cases one need not worry about cancellations between numerator and denominator of $\left.Q_{\lambda}\left(q^{n}, q\right)\right)$.

By Theorem $4.6 \pi_{\mathrm{tr}}$ is unitary if $\bar{Q}_{\lambda}(r, q) / x^{|\lambda|}$ is positive and $\pi_{\lambda}$ is a unitary representation of $H_{|\lambda|}\left(q^{2}\right)$ for all $\lambda \in \Gamma(r, q)$. Observe that for $q=e^{\pi i / l}$ one has the following identities;

and

$$
\frac{[n+d(i, j)]_{q}}{[h(i, j)]_{q}}=\frac{\sin ((n+d(i, j)) \pi / l)}{\sin (h(i, j) \pi / l)}
$$

$$
\frac{\left[n+\lambda_{i}-\lambda_{j}^{\prime}\right]_{q}+[h(j, j)]_{q}}{[h(j, j)]_{q}}=\frac{2 \sin \left(\left(n+2 \lambda_{j}-2 j+1\right) \pi / l\right) \cos \left(\left(n-2 \lambda_{j}^{\prime}+2 j-1\right) \pi / l\right)}{\sin (h(j, j) \pi / l)} .
$$

It is now easy to check for $2<n<l-2$ that for each $\lambda \in \Gamma\left(q^{n}, q\right)$ the factor corresponding to a box $(i, j)$ in $Q_{\lambda}\left(q^{n}, q\right)$ is positive. Similarly one checks in case (c) that each factor is negative, and, in particular, also $x=Q_{[1]}\left(q^{n}, q\right)$. Hence also 
$Q_{\lambda}\left(q^{n}, q\right) / x^{|\lambda|}>0$ for each $\lambda \in \Gamma\left(q^{n}, q\right)$. For (b), observe that it is enough to check the statement for diagrams with the additional assumption $\lambda_{1} \leqq(-1-n) / 2$ by Corollary 5.6(e) and remark (b). This is done as in case (c).

As all diagrams in $\Gamma(r, q)$ are eigher $l$ diagrams or $(k, l)$ diagrams, the corresponding representations of the Hecke algebras are unitary by [W-1, Proposition 2.10]. Hence also the second condition of Theorem 4.6 is satisfied.

The graphs $\Gamma\left(q^{n}, q\right)$ can be interpreted as truncated Weyl chambers. As examples, we sketch the graphs $\Gamma\left(q^{n}, q\right)$ for $n=3$ (related to $O(4)$ )

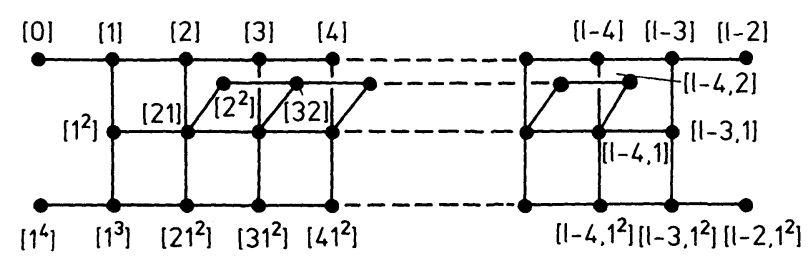

Fig. 8

and for $n=-5$ (with $[r, s]$ indicating the number of boxes in the first and second column of the diagrams) (related to $S p(4)$ ).

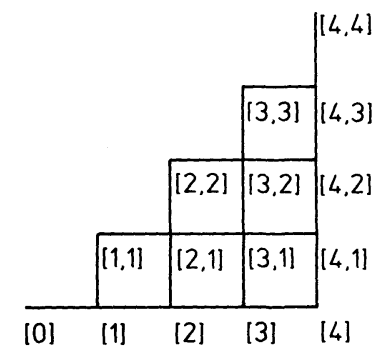

Fig. 9

\section{New Examples of Subfactors}

We can now apply the results above to construct more examples of irreducible subfactors using the same method as in [W-1]. We fix $m \in \mathbf{N}$ and $r, q \in \mathbf{C}$ such that $\pi_{\mathrm{tr}}$ is a unitary representation of $C_{\infty}(r, q)$. Let moreover $C_{m, f}$ and $C_{m, \infty}$ be the subalgebras of $C_{\infty}$ generated by $g_{m}, g_{m+1}, \ldots g_{f-1}$ and by $g_{m}, g_{m+1}, \ldots$ respectively. Then one defines for $f>m+1$ the $C^{*}$ algebras

$$
A_{f}=\pi_{\mathrm{tr}}\left(C_{m+1, f}(r, q)\right) \subset B_{f}=\pi_{\mathrm{tr}}\left(C_{f}(r, q)\right) .
$$

We denote by $\varepsilon_{A_{f}}$ and $\varepsilon_{B_{f}}$ the trace preserving conditional expectations onto $A_{f}$ and $B_{f}$ respectively. By Proposition 3.2 $A_{f+1}$ is the linear span of elements of the form $a_{1} \pi_{\mathrm{tr}}(\chi) a_{2}$ with $a_{1}, a_{2} \in A_{f}$ and $\chi \in\left\{g_{f}, e_{f}, 1\right\}$. Using this and the Markov property of $\operatorname{tr}($ Lemma $3.4(\mathrm{~d})$ ), one checks easily (see [W-1], Proposition 3.2) that

$$
\varepsilon_{A_{f+1}} \varepsilon_{B_{f}}=\varepsilon_{A_{f}}
$$


(7.2) is usually referred to as the "commuting square condition." Recall also that for $0 \leqq d<f$ the map $v_{d, f}: g_{i} \mapsto g_{f+d-i}, i=d+1, d+2, \ldots f-1$ extends to an inner automorphism of $C_{\infty}(r, q)$ (see Sect. 1 or [Bi]). In particular, we have (with $v_{f}=v_{0, f}$ )

$$
v_{f}\left(A_{f}\right)=B_{f-m} \text { and therefore } A_{f} \cong B_{f-m} .
$$

Observe that if $\Gamma(r, q)$ is a finite graph with depth $b$ (i.e. any vertex in $\Gamma(r, q)$ can be connected to [0] by at most $b$ edges of $\Gamma(r, q)), \pi_{\mathrm{tr}}\left(C_{f}(r, q)\right)$ is isomorphic to the basic construction for $\pi_{\mathrm{tr}}\left(C_{f-2}(r, q)\right) \subset \pi_{\mathrm{tr}}\left(C_{f-1}(r, q)\right)$ for $f \geqq b+1$. Hence the Bratteli diagram becomes periodic with inclusion matrices $G$ and $G^{t}$. It is not hard to see that $G$ is the matrix belonging to the inclusion diagram given by $\Gamma(r, q)$ with the diagrams with an even number of boxes in one line and the ones with an odd number in the other line. More generally, we say that the pair $\left(A_{f}\right) \subset\left(B_{f}\right)$ is periodic with periodicity $k$ if there exists an $f_{0} \in \mathbf{N}$ such that for all $f \geqq f_{0}$ the inclusion diagram

$$
\begin{gathered}
A_{f} \subset B_{f} \\
\cap \\
A_{f+1} \subset B_{f+1}
\end{gathered}
$$

is the same as the one for $A_{f+k}, A_{f+1+k}, B_{f+k}$ and $B_{f+1+k}$ (with a suitable bijection between the simple components of $A_{f}$ and $A_{f+k}, B_{f}$ and $B_{f+k}$, etc.).

It is well-known that under our periodicity assumptions there exists at most one normalized trace tr on $\bigcup_{f} B_{f}$, which, therefore, has to be a factor trace (i.e. the weak limit of $\pi_{\mathrm{tr}}\left(\bigcup_{f} B_{f}\right)$ is a factor). So if $\Gamma(r, q)$ is finite, one obtains a pair of hyperfinite $\mathrm{II}_{1}$ factors

$$
A=\pi_{\mathrm{tr}}\left(C_{m+1, \infty}(r, q)^{\prime \prime} \subset B=\pi_{\mathrm{tr}}\left(C_{\infty}(r, q)\right)^{\prime \prime} .\right.
$$

Theorem 7.1. Let $r, q \in \mathbf{C}$ be such that $\pi_{\mathrm{tr}}$ is a unitary representation of $C_{\infty}(r, q)$. With above notation one has for the subfactor $A \subset B$

and

$$
[B: A]=x^{2 m}=\left(\frac{r-r^{-1}+q-q^{-1}}{q-q^{-1}}\right)^{2 m}
$$

$$
A^{\prime} \cap B=B_{m}=\pi_{\mathrm{tr}}\left(C_{m}(r, q)\right) \text {. }
$$

In particular, if $m=1, A^{\prime} \cap B=\mathbf{C}$, i.e. the centralizer (or relative commutant) is trivial. The index values are listed in Table 1 for the various cases. Moreover, one obtains for each $\lambda \in \Gamma(r, q)$ an irreducible subfactor with index $Q_{\lambda}(r, q)^{2}$.

Proof. It follows from the discussion above that the algebras $A_{f}$ and $B_{f}$ satisfy the hypotheses for [W-1, Theorems 1.5 and 1.6]. Observe that the weight vector $\vec{t}_{f}$ of $\mathrm{tr}$ for $B_{f}$ is equal to $\left(Q_{\lambda}(r, q) / x^{f}\right)_{\lambda \in \Gamma_{f}(r, q)}$ and that the weight vector $\vec{s}_{f}$ for $A_{f}$ is equal to $\vec{t}_{f-m}$ by (7.3). Let $\|\cdot\|$ denote the usual $L^{2}$ norm. By [W-1], Theorem 1.5 one has

$$
[B: A]=\frac{\left\|\vec{s}_{f}\right\|^{2}}{\left\|\vec{t}_{f}\right\|^{2}}=\frac{\left\|\vec{t}_{f-m}\right\|^{2}}{\left\|\vec{t}_{f}\right\|^{2}} \text { for } f>d+m \text {. }
$$

Hence if $f>d+2 m$, one has

$$
[B: A]^{2}=\frac{\left\|\vec{t}_{f-m}\right\|^{2}}{\left\|\vec{t}_{f}\right\|^{2}} \frac{\left\|\vec{t}_{f-2 m}\right\|^{2}}{\left\|\vec{t}_{f-m}\right\|^{2}}=\frac{\left\|\vec{t}_{f-2 m}\right\|^{2}}{\left\|\vec{t}_{f}\right\|^{2}} .
$$


But as $f-2 m>d, \Gamma_{f-2 m}(r, q)=\Gamma_{f}(r, q)$. Hence it follows from Theorem 4.4 that the last quotient is equal to $x^{4 m}$ which shows the first claim.

It follows from the defining relations that $\pi_{\mathrm{tr}}\left(C_{m}(r, q)\right) \subset A^{\prime} \cap B$. On the other hand, choose $f \in \mathbf{N}$ such that $f$ is even and $f>d$ and choose a minimal projection $p \in B_{f,[0]}$. Now observe that the Bratteli diagram for

$$
p B_{f} p \subset p B_{f+1} p \subset p B_{f+2} p \subset \ldots \subset p B_{f+m} p
$$

is exactly the same as the one for

$$
B_{0} \subset B_{1} \subset B_{2} \subset \ldots \subset B_{m} .
$$

Hence $\operatorname{dim} p B_{f+m} p=\operatorname{dim} B_{m}$. Observe that the inner automorphism $v_{f+m}$ maps $B_{f} \subset B_{f+m}$ onto $A_{f+m}$. Hence one also has $\operatorname{dim} v_{f+m}(p) B_{f+m} v_{f+m}(p)=\operatorname{dim} B_{m}$. But then it follows from [W-1], Theorem 1.6 that

$$
\operatorname{dim} A^{\prime} \cap B \leqq \operatorname{dim} B_{m}
$$

which shows the second claim.

Let $\lambda \in \Gamma(r, q)$. Choosing $m$ appropriately (e.g. $m=|\lambda|)$, there exists a pair of factors $A \subset B$ such that $A^{\prime} \cap B=B_{m}$ and $B_{m}$ contains a simple component labelled by $\lambda$. Let $p$ be a minimal projection in that component. As $p$ is minimal one has $A_{p}^{\prime} \cap B_{p}=\mathbf{C}$ and by [W-1], Theorem 1.5 (iii)

$$
\left[B_{p}: A_{p}\right]=\operatorname{tr}(p)^{2}[B: A]=\frac{Q_{\lambda}^{2}(r, q)}{x^{2 m}} x^{2 m}=Q_{\lambda}^{2}(r, q)
$$

To obtain finer invariants besides index and centralizer, one also considers the "higher centralizers" or "higher relative commutants" defined in the following way:

Let $A \subset B$ be $\mathrm{II}_{1}$ factors with finite index. Then one defines a sequence $B^{(i)}$ of $\mathrm{II}_{1}$ factors inductively by $B^{(0)}=A, B^{(1)}=B$ and for $i>1, B^{(i)}$ is the basic construction for $B^{(i-2)} \subset B^{(i-1)}$ with generating projection $\bar{e}_{i-1}$. Here one uses the fact that $B^{(i)}$ is also a finite factor (see [Jo-1, Sect. 3]), hence it has a unique normalized trace which extends $\operatorname{tr}$ and is also denoted by tr. If $\varepsilon_{B}\left(\right.$ i) is the conditional expectation onto $B^{(i)}$, one also has

$$
\varepsilon_{B}{ }^{(i)}\left(\bar{e}_{i}\right)=\operatorname{tr}\left(\bar{e}_{i}\right)=1 /[B: A] .
$$

Then the higher centralizers (or higher relative commutants) $Z_{i}$ are defined by

$$
Z_{i}=A^{\prime} \cap B^{(i)}
$$

A subfactor is said to be of finite depth if the dimension of the centers of the $Z_{i}$ 's is uniformly bounded. It is well-known that in this case there exists an $i_{0}$ such that $Z_{i+1}$ is isomorphic to Jones' basic construction for $Z_{i-1} \subset Z_{i}$ for all $i>i_{0}$ (it can for instance be deduced from [W-1, Corollary 1.2]). This definition is due to Ocneanu who also announced that for such subfactors the higher relative commutants of the factor and the subfactor completely describe the original subfactor (see [O] or [Po] for a more precise statement). This has been proven recently by Popa in [Po].

Due to the fact that the basic construction already occurs naturally within the algebras $C_{f}$ it is not difficult to compute the higher relative commutants for our examples. 
Corollary 7.2. Let $A \subset B$ be a pair of factors constructed in the previous theorem with $m=1$. Then

$$
Z_{i}=B_{i}
$$

In particular, all our constructed subfactors are of finite depth.

Proof. As all $g_{j}$ 's are conjugate to each other, the shift $\mathrm{sh}_{i-1}: g_{j} \mapsto g_{j+i-1}$ extends to a trace preserving injective homomorphism from $C_{\infty}$ into itself. This can be extended to an isomorphism $\mathrm{sh}_{i-1}$ mapping $A$ onto $\pi_{\mathrm{tr}}\left(C_{i+1, \infty}(r, q)^{\prime \prime}\right.$ and $B$ onto $\pi_{\mathrm{tr}}\left(C_{i, \infty}(r, q)\right)^{\prime \prime}$. By Theorem 7.1, it is enough to show that $\operatorname{sh}_{i-1}$ can be extended to an isomorphism from $B^{(i)}$ onto $\pi_{\mathrm{tr}}\left(C_{\infty}\right)$.

Let $i=2$ and let $\bar{e}_{1}$ be the projection obtained from the basic construction for $A \subset B$. It follows from the periodicity and [W-1], Theorem 1.5 (ii) that for $f$ large enough the algebra generated by $B_{f}$ and $\bar{e}_{1}$ is isomorphic to the basic construction for $A_{f} \subset B_{f}$.

But on the other hand, by the finiteness of the graph $\Gamma(r, q)$ the algebra generated by $\pi_{\mathrm{tr}}\left(e_{1}\right)$ and $B_{f}$ is also isomorphic to $\pi_{\mathrm{tr}}\left(C_{f}(r, q)\right)$ for $f$ big enough (just use Theorem 4.4 and the automorphism $\left.\eta_{f+1}\right)$. By Theorem 4.4, the isomorphism can be realized by mapping $\bar{e}_{1}$ to $(1 / x) e_{1}$ and it is easy to check that it is trace preserving by Lemma 3.4 (d) and the Markov property (see [Jo-1, Proposition 3.1.7]). As this is true for all $f \in \mathbf{N}$ which are large enough the isomorphism can be extended to the corresponding von Neumann algebras.

The general case follows from this by induction on $i$. Indeed, it is enough to apply $\mathrm{sh}_{1}$ to the case $i-1$ and then to repeat the step above for $B^{(i-2)} \subset B^{(i-1)}$ instead of $A \subset B$.

\section{Table 1}

\begin{tabular}{|c|c|c|c|c|}
\hline$n$ & $l$ & $\Gamma\left(q^{n}, q\right)$ & positivity & index \\
\hline 0 & Arbitrary & $\begin{array}{cc}{[0]} & {[1]} \\
0 & 0\end{array}$ & arbitrary & 1 \\
\hline 1 & Arbitrary & $D_{\infty}$ & arbitrary & 4 \\
\hline 2 & $l \in \mathbb{N}$ & see Prop. 6.1 & $e^{2 \pi i / l}$ & $\frac{\sin ^{2} 3 \pi / l}{\sin ^{2} \pi / l}$ \\
\hline$n>2$ & $l \in \mathbb{N}$ & $\begin{array}{c}\left\{\lambda, \lambda_{1}+\lambda_{2} \leqq l-n+1\right. \\
\left.\lambda_{1}^{\prime}+\lambda_{2}^{\prime} \leqq n+1\right\} \\
\cup\left\{\left[l-n+1,1^{n-1}\right]\right\}\end{array}$ & $e^{\pi i / l}$ & $\left(\frac{\sin n \pi / l}{\sin \pi / 1}+1\right)^{2}$ \\
\hline $\begin{array}{l}n<-1 \\
n \text { even }\end{array}$ & $\begin{array}{l}l \in \mathbb{N} \\
l \text { odd }\end{array}$ & $\begin{aligned}\{\lambda, & \lambda_{1}+\lambda_{2} \leqq-1-n \\
& \left.\lambda_{1}^{\prime} \leqq(l+n-1) / 2\right\}\end{aligned}$ & $e^{\pi i / l}$ & $\left(\frac{\sin |n| \pi / l}{\sin \pi / l}-1\right)^{2}$ \\
\hline $\begin{array}{l}n<-1 \\
n \text { odd }\end{array}$ & $\begin{array}{l}l \in \mathbb{N} \\
l \text { even }\end{array}$ & $\left\{\begin{aligned}\{\lambda, & \lambda_{1} \leqq(-1-n) / 2 \\
& \left.\lambda_{1}^{\prime} \leqq(l+n-1) / 2\right\}\end{aligned}\right.$ & $e^{\pi i / l}$ & $\left(\frac{\sin |n| \pi / l}{\sin \pi / l}-1\right)^{2}$ \\
\hline
\end{tabular}


In Table 1 we give a survey of unitarizable representations of $C_{\infty}$. More precisely, $\pi_{\mathrm{tr}}$ is unitarizable if $q$ has the value indicated in the column "positive". The last column lists the index of the corresponding subfactor $\pi_{\mathrm{tr}}\left(C_{2, \infty}(r, q)\right)^{\prime \prime} \subset \pi_{\mathrm{tr}}\left(C_{\infty}(r, q)\right)^{\prime \prime}$.

\section{Concluding Remarks}

1. There is a general procedure to construct link invariants and subfactors for each irreducible representation of a classical Lie group (see also [ $\mathrm{T}$ and $\mathrm{Re}$ ]).

(a) Starting with a representation $\varrho$ of a Lie group $G$, consider the centralizers of tensor products of its $q$ deformation. This will always contain representations of the braid groups.

(b) Let $D$ be the $q$ power of the diagonal matrix (we assume the Cartan algebra to be in diagonal form) corresponding to half the sum of all the positive roots of the Lie algebra of $G$ with respect to a suitably normalized invariant bilinear form (see e.g. the matrix $D$ defined above (5.6) or, in general, [Re or Pi]). The corresponding product "state" defines a trace on the centralizer algebras with Markov property, i.e. a trace which can be used to define a link invariant by Jones' approach (see [Jo-2] or, in a slightly more general form [W-3]).

(c) Determine the image of the centralizer algebras under the representation coming from the GNS construction and determine when these representations are unitarizable.

(d) If, in the unitary case, the Markov trace satisfies an additional condition, the socalled commuting square condition (7.2), one obtains the subfactor $\pi_{\mathrm{tr}}\left(B_{2, \infty}\right)^{\prime \prime} \subset \pi_{\mathrm{tr}}\left(B_{\infty}\right)^{\prime \prime}$. Its index is given by the square of the $q$ dimension of the representation $\varrho$.

It should be notized that in general the centralizer algebra is larger than the algebra generated by the representation of the braid group (see also remarks about $S O(2 k)$ in 3 and 4$)$. Similarly as one can obtain from a given representation $\varrho$ of a group new representations by taking tensor products, one can, at least in principle, derive the corresponding link invariants and braid representations via cabling (see [M-2] and [W-3]). The subfactor corresponding to the $f^{\text {th }}$ tensor power of $\varrho$ is more conveniently obtained by considering $\pi_{\mathrm{tr}}\left(B_{f+1, \infty}\right)^{\prime \prime} \subset \pi_{\mathrm{tr}}\left(B_{\infty}\right)^{\prime \prime}$. Although it seems very likely, it is not clear at the moment whether this subfactor is isomorphic to the one obtained from the cabling procedure.

2. We list in the table below the cases for which the program described in 1 . has been carried out. We remark that most of the objects in that table were originally obtained by different methods (e.g. Jones' subfactors and his polynomial, Hecke algebra subfactors, Kauffman polynomial). The corresponding braid representations can also be described in terms of loop groups (see remarks below). For a more direct approach see [Ko]. It would be interesting to find out whether one can also construct subfactors from Reshetikhin's $R$ matrices corresponding to spin representations (see $[\mathrm{Re}]$ ). Finally observe that we have also computed the indices of subfactors corresponding to irreducible representations which occur in some tensor power of the standard representation. It also seems likely that one can compute their higher relative commutants by computing branching coefficients similar as in [GW] (see also Remark 6). 
Table 2

\begin{tabular}{llll}
\hline Lie group & Braid representation & Link invariant & Subfactors \\
\hline$S U(2)$ & Temperley-Lieb algebras & Jones polynomial & Jones' subfactors \\
\hline$S U(k)$ & Hecke algebras & FYHLMO polynomialSee [W-1] \\
\hline$O(k), S p(k)$ & $C_{f}\left(q^{ \pm k-1}, q\right)$ & Kauffman polynomial See Theorem 7.1 \\
\hline Tensor products & Cabled representations & Cabled invariants & $\begin{array}{l}\text { Subfactors } \\
\pi_{\mathrm{tr}}\left(B_{f+1, \infty}\right)^{\prime \prime} \subset \pi_{\mathrm{tr}}\left(B_{\infty}\right)^{\prime \prime}\end{array}$ \\
\hline
\end{tabular}

3. Similarly as for our algebras, one can also obtain the defining relations of the Hecke algebras from skein relations, namely from those of the Homfly polynomial in [FYHLMO]. The proof is essentially the same as the one for Lemma 3.1 except that one need not even worry about the subtleties coming from regular isotopy (i.e. one can define the trace directly from the link invariant). Somewhat amazingly, the classification of all unitary representations of the inifinite braid group which factor through these algebras, leads to the truncated Weyl chambers occurring for most of the classical loop groups (see [W-1] and below) and to structure coefficients as the fusion rules in the corresponding Wess-Zumino-Witten models (see [GW] and 4).

The graphs $\Gamma(r, q)$ which describe the unitary quotients of our algebras (see Theorem 6.4) correspond to truncated Weyl chambers of the full orthogonal, the odd dimensional orthogonal (mod highest root of the symplectic) and the symplectic groups. More precisely, the vertices are in 1-1 correspondence with the highest weights in a Weyl chamber, which is truncated by a hyperplane determined by the highest root of the same groop (except case (b); see above). Similar restrictions were obtained in [JMO] by constructing explicit solutions of the star triangle relation. This method is similar, but more general than our construction of representations of Hecke algebras (see also [Pa]). Similar results were also described in [AGS].

4. Let us briefly sketch how one can obtain the coefficients for the fusion rules of WZW models in this context see [GW]. The method consists of decomposing products of minimal idempotents in $\pi\left(C_{f}(r, q)\right)$ and $\pi\left(C_{f+1, m}(r, q)\right)$ into a sum of minimal idempotents of $\pi\left(C_{m}(r, q)\right.$ ) (where $\pi$ is a unitary representation of $\left.C_{\infty}(r, q)\right)$. Such a decomposition is, in general, not unique, but the number of idempotents which are in a given simple component of $\pi\left(C_{m}(r, q)\right)$ does not depend on the chosen decomposition. These coefficients have been computed in [GW] for type $A$ (i.e. for braid representations into the Hecke algebras) in terms of the classical Littlewood-Richardson coefficients and an affine reflection group, coming from the Weyl group and the reflection about a hyperplane given by the highest root of $S U(k)$. The same formula can be obtained for the coefficients of fusion rules (see e.g. [Kc, ex. 13.34, 35]). One would expect a similar result for the representations constructed here.

5. If $q$ tends to 1 , the characteristic graph becomes in the limit a graph describing the fixed point algebra of a product type action of an irreducible representation of a symplectic or full orthogonal group which, for the standard representations, is essentially just the Weyl chamber without the weights belonging to spin repre- 
sentations. The corresponding subfactors have been studied by Antony Wassermann in [Wa] and unpublished work. In this paper, we do not obtain in the classical limit the subfactors arising from product type actions of the special even dimensional orthogonal group. For this, one presumably would have to consider a $q$ deformation of the centralizer algebra of $S O(2 n)$, i.e. one would have to introduce additional generators corresponding to a $q$ deformation of the Pfaffian. This might also clarify the somewhat mysterious fact that the Coxeter graphs $D_{2 n}$ (but not $D_{2 n+1}$ ) occur in Ocneanu's list of subfactors with index less than 4 (which are not among the examples constructed here).

6. Finally let us also mention an analogy between Jones' basic construction and taking tensor products of representations of Lie groups. This is actually most explicitly seen for Antony Wassermann's examples coming from product type actions of symplectic and orthogonal groups. It follows from his results (see [Wa]) that the $f^{\text {th }}$ higher relative commutant of such a subfactor is isomorphic to the centralizer of the $f$-fold tensor product of the corresponding representation (if it is self conjugate). So the coefficients obtained by the approach in [GW] could be considered as structure constants for this analogy of tensor products for subfactors with $q$ a root of unity.

It can be shown that for the subfactors coming from the Hecke algebras (see [W-1]), the higher relative commutants do not reproduce the generating algebras. In the limiting case $q=1$ this follows from the fact that representations of Lie algebras of type $A_{k}, k>1$ are not self-conjugate (see [Wa]). The coefficients in [GW] can only be obtained if one considers the centralizer algebras of the shifted subfactors, obtained by iterating the map $s h: B_{\infty} \rightarrow B_{\infty}$ given by $\sigma_{i} \mapsto \sigma_{i+1}$. It is, in this context, possible to define tensor products $B^{\otimes n}=B \otimes_{A} B \otimes_{A} \ldots \otimes_{A} B$ (notation as in Theorem 7.2) with a multiplicative structure such that $A \subset B^{\otimes n}$ is isomorphic to $\pi_{\mathrm{tr}}\left(B_{n+1, \infty}\right)^{\prime \prime} \subset \pi_{\mathrm{tr}}\left(B_{\infty}\right)^{\prime \prime}$ and where the braid group appears naturally in the commutant of $A$ in this extension. This new extension, which can be thought of as an inverse construction of the shift, will be discussed somewhere else.

Acknowledgements. The author would like to thank the IHES, the Mittag-Leffler Institute, Odense University and University of Geneva for support.

\section{References}

[AGS] Alvarez-Gaume, L., Gomez, C., Sierra, G.: Duality and quantum groups, preprint, CERN

[B] Birman, J.: Braids, link and mapping class groups. Ann. Math. Studies vol. 82, Princeton, NJ: Princeton University Press 1974

[BW] Birman, J., Wenzl, H.: Braids, link polynomials and a new algebra. Trans. AMS 313, 249-273 (1989)

[Bk] Bourbaki, N.: Groupes et Algèbres de Lie, IV-VI

[Br] Brauer, R.: On algebras which are connected with the semisimple continuous groups. Ann. Math. 63, 854-872 (1937)

[DJ1] Dipper, R., James, G.: Repr. of Hecke algebras of the general linear group. Proc. LMS 52, 20-52 (1986)

[DJ2] Dipper, R., James, G.: Blocks and idempotents of the Hecke algebra of the general linear group. Proc. LMS 54, 57-82 (1987)

[D] Drinfeld, V.: Quantum groups, Proceedings for the ICM Berkeley, 1986, pp. $798-820$ 
[EK] El Samra, N., King, R.: Dimensions of irreducible representations of the classical Lie groups. J. Phys. A: Math. Gen. 12, 2317-2328 (1979)

[FRS] Fredenhagen, K., Rehren, K.H., Schroer, B.: Superselection sector with braid statistic and exchange algebra, General Theory. Commun. Math. Phys. 125, 201-226 (1989)

[FYHLMO] Freyd, P., Yetter, D., Hoste, J., Lickorish, W.B.R., Millett, K., Ocneanu, A. : A new polynomial invariant of knots and links. Bull. AMS 12, No. 2, 239-246 (1985)

[FFK] Felder, G., Fröhlich, J., Keller, G.: Braid matrices and structure constants for minimal conformal models, Commun. Math. Phys. 124, 647-664 (1990)

[GM] Garsia, A., McLarnan, T.: Relations between Young's natural and the KazhdanLusztig representations of $S_{n}$. Adv. Math. 69, 32-92 (1988)

[GW] Goodman, F., Wenzl, H.: Littlewood Richardson coefficients for Hecke algebras at roots of unity. Adv. Math (1990)

[G] Gyoja, A.: A q-analogue of Young symmetrizer. Osaka J. Math. 23, 841-852 (1986)

[HS] Haagerup, U., Schou, J.: Examples of subfactors, preprint Odense University

$[\mathrm{H}] \quad$ Hoefsmit, P.N.: Representations of Hecke algebras of finite groups with BN pairs of classical type, thesis, University of British Columbia (1974)

[Ji-1] Jimbo, M.: Quantum $R$-matrix for the generalized Toda system. Commun. Math. Phys. 102, 537-547 (1986)

[Ji-2] Jimbo, M.: A $q$ analogue of $U(g l(N+1))$, Hecke algebras and the Yang-Baxter equation. Lett. Math. Phys. 10, 63-69 (1985)

[Ji-3] Jimbo, M.: Introduction to the Yang-Baxter equation. Braid Group, Knot Theory and Statistical Mechanics, pp. 111-134. World Scientific (1989)

[JMO] Jimbo, M., Miwa, T., Okado, M.: Solvable lattice models related to the vector representations of classical simple Lie algebras. Commun. Math. Phys. 116, 507-525 (1988)

[Jo-1] Jones, V.F.R.: Index for subfactors. Invent Math. 72, 1-25 (1983)

[Jo-2] Jones, V.F.R.: Hecke algebra representations of braid groups and link polynomials. Ann. Math. 126, 335-388 (1987)

[Jo-3] Jones, V.F.R.: On knot invariants related to some statistical mechanics models. Pacific J. Math. (1989)

[Kc] Kac, V.: Infinite dimensional Lie algebras, 3rd edition

[K] Kauffman, L.H.: An invariant of regular isotopy. Trans. AMS 318, 417-471 (1990)

[KL] Kazhdan, D., Lusztig, G.: Representations of Coxeter groups and Hecke algebras. Invent Math. 53, 165-184 (1979)

[Ko] Kohno, T.: Ann. Inst. Four.

$[\mathrm{L}]$

[MW]

Lusztig, G.: Quantum deformations of certain simple modules over enveloping algebras. Adv. Math. 70, 237-249 (1988)

$[\mathrm{M}-2]$

$[\mathrm{O}]$ Morton, H., Wassermann, A.: A basis for the Birman-Wenzl algebra, preprint Murakami, J.: The Kauffman polynomial of links and representation theory. Osaka J. Math. 24, 745-758 (1987)

Murakami, J.: The parallel version of link invariants, preprint

Ocneanu, A.: Quantized groups, string algebras and Galois theory for algebras. Lond. Math. Soc. Lect. Notes vol. 136, Evans/Takesaki (ed.) pp. 119-172

[PP] Pimsner, M., Popa, S.: Entropy and index for subfactors. Ann. Sci. Ec. Norm. Sup. 19, 57-106 (1986)

[Po] Popa, S.: On the classification of subfactors. Invent. Math. 101, 19-43 (1990)

[RW] Ram, A., Wenzl, H.: Matrix units for centralizer algebras. J. of Algebras (to appear)

[Re] Reshetikhin, N.Yu.: Quantized universal enveloping algebras, the Yang-Baxter equation and invariants of links, LOMI preprint

[Ro] Rosso, M.: Finite dimensional representations of the quantum analog of the enveloping algebra of a complex simple Lie algebra. Commun. Math. Phys. 117, 581-593 (1988)

[T] Turaev, V.G.: The Yang-Baxter equation and invariants of links, Invent. Math.92, $527-553(1988)$ 
[Wa] Wassermann, A.: Coactions and Yang-Baxter equations for ergodic actions and subfactors, Lond. Math. Soc. Lect. Notes 136, Evans/Takesaki (ed.), pp. 203-236

[W-1] Wenzl, H.: Hecke algebras of type $A_{n}$ and subfactors, Invent Math. 92, 349-383 (1988)

[W-2] Wenzl, H.: On the structure of Brauer's centralizer algebras, Ann. Math. 128, 173193 (1988)

[W-3] Wenzl, H.: Representations of braid groups and the quantum Yang-Baxter equation. Pacific J. Math. (1990)

[W-4] Wenzl, H.: Unitarizations of solutions of the quantum Yang-Baxter equation and subfactors. Proc. Congress IAMP, Swansea, 1988 (to appear)

[W-5] Wenzl, H.: Quantum groups and subfactors of Lie type B, C and D, preprint

[Wy] Weyl, H.: The classical groups. Princeton, NJ: Princeton University Press

[Y] Yamane, H.: Irreducible projective modules of the Hecke algebras of a finite Coxeter group, preprint, Osaka University

Communicated by N. Yu. Reshetikhin 